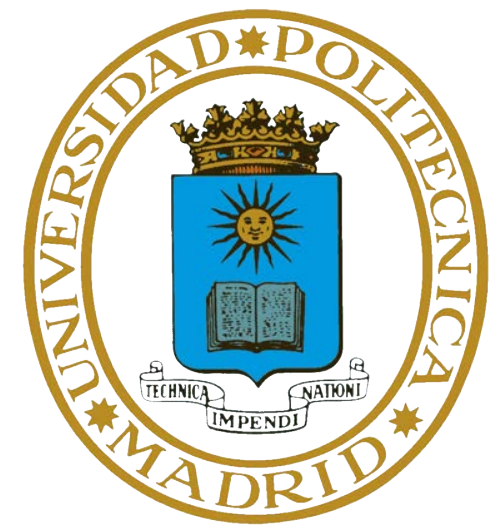

ESCUELA TÉCNICA SUPERIOR DE INGENIEROS INFORMÁTICOS

Tools for the Detection and Analysis of Potentially Unwanted Programs

\author{
PH.D THESIS
}

Richard Rivera Guevara 
Copyright $\bigcirc 2018$ by Richard Rivera Guevara 
DEPARTAMENTAMENTO DE LENGUAJES Y SISTEMAS INFORMÁTICOS E INGENIERIA DE SOFTWARE

ESCUELA TÉCNICA SUPERIOR DE INGENIEROS INFORMÁtICOS

\title{
Tools for the Detection and Analysis of Potentially Unwanted Programs
}

\author{
SUBMITTED IN PARTIAL FULFILLMENT OF THE REQUIREMENTS FOR THE DEGREE OF: \\ Doctor of Philosophy in Software, Systems and Computing
}

Author: Richard Rivera Guevara

Advisor

Dr. Juan Caballero

2018 


\section{Abstract}

In this thesis we study potentially unwanted programs (PUP), a category of undesirable software that, while not outright malicious, contains behaviors that may alter the security state or the privacy of the system on which they are installed. PUP often comes bundled with freeware, i.e., proprietary software that can be used free of charge. A popular vector for distributing freeware are download portals, i.e., websites that index, categorize, and host programs. Download portals can be abused to distribute PUP. Freeware is often distributed as an installer, i.e., an auxiliary program in charge of performing all installation steps for the target program. During installation, besides the target program desired by the user, the installer may install PUP as well. PUP may be difficult to uninstall and may persist installed in the system after the user tries to uninstall it. Current malware analysis systems are not able to detect and analyze characteristic behaviors of PUP. For example, current malware analysis systems operate on a single program execution, while detecting incomplete PUP uninstallations requires analyzing together two program executions: the installation and the uninstallation.

This thesis presents novel tools to detect and analyze potentially unwanted programs. More concretely, it describes three main contributions. First, it presents a measurement study of PUP prevalence in download portals, exposing the abusive behaviors that authors of malicious software use to distribute their applications through download portals. Second, it proposes a system especially designed to dynamically detect and analyze PUP behaviors during program installation and uninstallation. Third, it describes AVCLASS, an automatic labeling tool that given the AV labels for a potentially massive number of samples, outputs the most likely family for each sample.

To analyze the distribution of PUP through download portals, we build a platform to crawl download portals and apply it to download 191K Windows freeware installers from 20 download portals. We analyze the collected installers measuring an overall ratio of PUP and malware between $8 \%$ (conservative estimate) and 26\% (lax estimate). In 18 of the 20 download portals examined the amount of PUP and malware is below 9\%. But, we also find two download portals exclusively used to distribute PPI 
downloaders. We also detail different abusive behaviors that authors of undesirable programs use to distribute their programs through download portals.

We present a platform to perform dynamic behavioral analysis of an input installer. Our platform executes the installer, navigates it to complete a successful installation, analyzes the installation to identify PUP behaviors, identifies the list of installed programs regardless of the installation location, checks whether each installed program has a corresponding uninstaller, executes the uninstallers, analyzes the uninstallation to identify PUP behaviors, and correlates the installation and uninstallation executions to determine if all installed programs were completely uninstalled.

Finally, we describe AVCLASS, a tool for automatically labeling malicious executables as variants of known families. AVCLASs takes as input the AV labels for a potentially massive number of samples and outputs the most likely family names for each sample. While AV labels are well-known to be inconsistent, there is often no other information available for labeling. AVCLASS implements novel automatic techniques to address 3 key challenges due to AV labels inconsistencies: normalization, removal of generic tokens, and alias detection. We have evaluated AVCLASS on 10 datasets comprising 8.9 M samples. AVCLASS's achieves F1 measures up to 93.9 on labeled datasets and samples are labeled with fine-grained family names commonly used by the AV vendors. We have released AVCLASS to the community. 
Esta tesis estudia los programas potencialmente no deseados (PUP), una categoría de software que, aunque no totalmente malignos, presentan comportamientos que pueden alterar la seguridad o la privacidad de los sistemas en que se instalan. El PUP suele venir empaquetado con freeware, i.e., software propietario que puede usarse sin coste. Un vector popular de distribución del freeware son los portales de descargas, i.e., sitios web que indexan, clasifican y alojan programas. Los portales de descargas pueden ser abusados para distribuir PUP. El freeware suele distribuirse como un instalador, i.e., un programa auxiliar encargado de realizar todos los pasos necesarios para instalar otro programa. Durante la instalación, además de instalar el programa deseado por el usuario, el instalador puede también instalar PUP. El PUP puede ser difícil de desinstalar y puede permanecer en el sistema después de que el usuario intente desinstalarlo. Los sistemas actuales de análisis de malware no son capaces de detectar comportamientos característicos del PUP. Por ejemplo estos sistemas operan sobre una sola ejecución de un programa, mientras que la detección del PUP suele requerir analizar juntas dos ejecuciones: la instalación y la desintalación.

Esta tesis presenta nuevas técnicas para detectar y analizar PUP y contiene tres contribuciones principales. Primero, se presenta un estudio de la prevalencia de PUP y malware en portales de descargas, exponiendo los comportamientos abusivos que utilizan sus autores. Segundo, se propone un sistema especialmente diseñado para identificar dinámicamente comportamientos de PUP durante la instalación y desintalación. Tercero, se describe AVCLASS, una herramienta automática de etiquetado, que dada las etiquetas asignadas por los antivirus (AV) a un número potencialmente masivo de muestras, identifica el nombre de familia más probable para cada muestra.

Para analizar la distribución de PUP a través de los portales de descargas construimos una plataforma y la usamos para descargar $191 \mathrm{~K}$ instaladores de freeware para Windows desde 20 portales. Analizando los instaladores medimos una proporción global de PUP y malware entre $8 \%$ (estimación conservadora) y 26\% (estimación laxa). En 18 de los 20 portales examinados, la cantidad es inferior al 9\%. Pero, también 
encontramos dos portales utilizados exclusivamente para distribuir PUP. Además, detallamos los diferentes comportamientos abusivos utilizados por los autores del PUP. A continuación, presentamos una plataforma para analizar dinámicamente instaladores. Nuestra plataforma ejecuta el instalador, lo navega para completar la instalación, analiza la instalación identificando comportamientos de PUP, identifica la lista de programas instalados, revisa si cada programa instalado tiene un desinstalador, ejecuta los desinstaladores, analiza la desintalación identificando comportamientos de PUP, y compara la instalación y desinstalación determinando si todos los programas se desinstalaron completamente.

Finalmente, describimos AVCLASS, una herramienta automática para etiquetar ejecutables maliciosos como variantes de familias conocidas. AVCLASS toma como entrada etiquetas asignadas por los $\mathrm{AV}$ de un número de muestras potencialmente masivo, e identifica la familia más probable para cada muestra. Aunque las etiquetas que asignan los AV suelen ser inconsistentes, a menudo no hay otra información disponible para el etiquetado. AVCLASS implementa novedosas técnicas automáticas para abordar tres desafíos debidos a la inconsistencia de las etiquetas de los AV: normalización, eliminación de tokens genéricos y detección de alias. Hemos evaluado AVCLASS en 10 datasets con 8,9M de muestras. AVCLASS alcanza una medida F1 de hasta 93.9 en datasets etiquetados y asigna nombres de familia comúnmente utilizados por los AV. Hemos puesto AVCLASS a disposición de la comunidad. 
To my mother Marlene,

my sister Jennifer,

and to the memory of my grandfather Ricardo. 


\section{Acknowledgments}

Firstly and foremost, I would like to express my sincere gratitude to my advisor Dr. Juan Caballero for the opportunity he gave to me to join his research group and for his patience and support. He has been a reliable source of ideas, guidance, and knowledge. I would like to also thank the people from the IMDEA Software Institute, especially to the Malicia Lab research team: Platon, Srdjan, Irfan, Antonio, and Avinash. I could always count on your support and understanding.

Next, I want to thank my family in Spain Ricardo and Estefy. You made it easy to deal with my constant homesick. Special thanks to Verónica for your support during this journey, and our recurrent conversations which helped me to clarify my ideas to improve my projects. Also, thanks to all my family in Ecuador, especially to Beatriz, Fernando, Fernanda, Carlos, et al. Your messages of encouragement always came just in the right moments.

Finally, I want to express my greatest gratitude to my mother and my sister Jennifer for encouraging me to pursue a PhD. Despite having spent difficult times, I could always count on your unconditional love and support. You were my greatest motivation to complete this work. 
1 Introduction $\quad 1$

1.1 PUP Detection . . . . . . . . . . . . . . . . 3

1.2 Measuring PUP and Malware Distribution in Download Portals . . . . 4

1.3 PUP and Malware Labeling . . . . . . . . . . . . . . . . . . . . 4

1.4 Thesis Contributions . . . . . . . . . . . . . . . 5

1.5 Thesis Outline . . . . . . . . . . . . . . . 6

2 Related Work $\quad 7$

2.1 PUP Distribution Through Download Portals . . . . . . . . . . . 7

2.2 PUP Detection Through Installation and Uninstallation Behaviors . . 8

2.3 PUP and Malware Labeling . . . . . . . . . . . . . . . . . 9

3 PUP Distribution Through Download Portals 12

3.1 Introduction . . . . . . . . . . . . . . . . 12

3.2 Download Portals . . . . . . . . . . . . . . . . . . 14

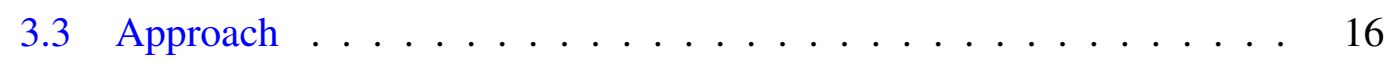

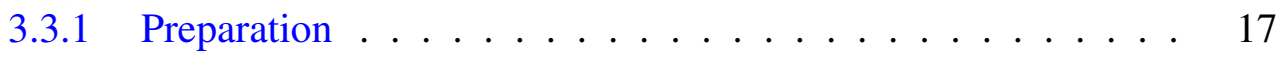

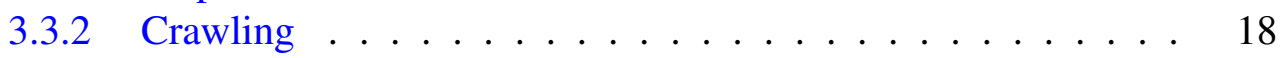

3.3 .3 File Processing . . . . . . . . . . . . . . . . 19

3.3 .4 Execution ........................ 20

3.4 Evaluation . . . . . . . . . . . . . . . . . 21

3.4.1 Download Portals Crawling Statistics . . . . . . . . . . 21

3.4.2 Undesirable Programs in Download Portals . . . . . . . . 23

3.4 .3 Abusive Behaviors ................. 24

3.4.4 Program Execution . . . . . . . . . . . . . . 26

3.4.5 Case Study: Vittalia Download Portals . . . . . . . . . . . 27 
4 PUP Detection through Installation and Uninstallation Behaviors 29

4.1 Introduction . . . . . . . . . . . . . . . . . . . . 29

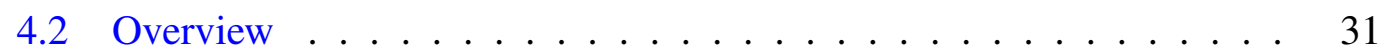

4.2.1 Installer Builder Software . . . . . . . . . . . . 31

4.2.2 Installation Monetization . . . . . . . . . . . . . 32

4.2.3 Enabling User Uninstallation . . . . . . . . . . . . . . 33

4.2 .4 PUP detection . . . . . . . . . . . . . . . 34

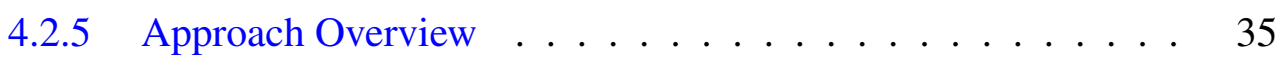

4.3 Program Execution . . . . . . . . . . . . . . . . . . 35

4.3.1 Automated User Interface Interaction . . . . . . . . . . 36

4.3.2 Behavioral Information . . . . . . . . . . . . . 36

4.3.3 Installer Classification . . . . . . . . . . . . 37

4.4 Identifying Installed Programs . . . . . . . . . . . . . . . . . . . 38

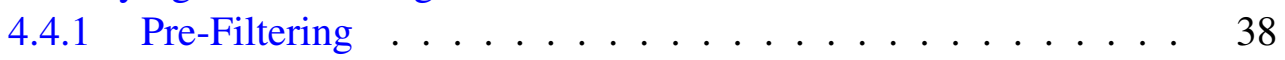

4.4 .2 Partitioning . . . . . . . . . . . . . 38

4.4.3 Identifying Uninstallation Commands . . . . . . . . . . 39

4.4 .4 Merging . . . . . . . . . . . . . . . 40

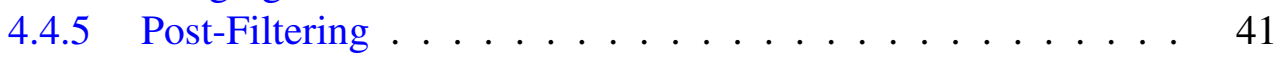

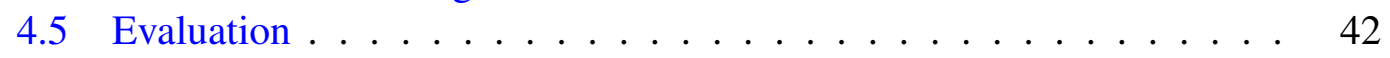

4.5 .1 Datasets ....................... 42

4.5.2 Installation Runs . . . . . . . . . . . . . . . . . . . 43

4.5 .3 Uninstallation Runs . . . . . . . . . . . . . . . . 44

4.6 Discussion . . . . . . . . . . . . . . . . . 45

$5 \quad$ PUP and Malware Labeling $\quad 46$

5.1 Introduction . . . . . . . . . . . . . . 46

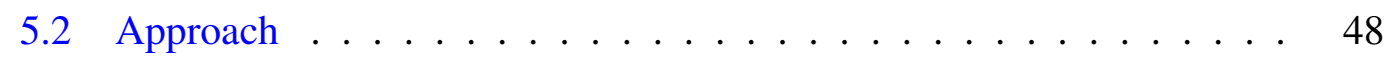

5.2 .1 Labeling . . . . . . . . . . . . . . . . 49

5.2 .2 Generic Token Detection . . . . . . . . . . . 53

5.2 .3 Alias Detection . . . . . . . . . . . . . 54

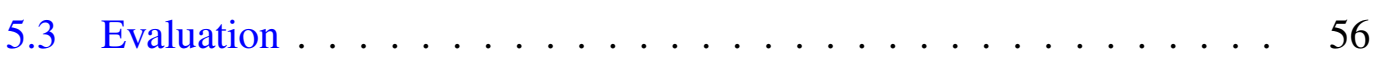

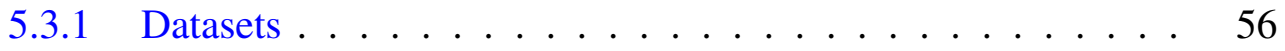

5.3 .2 Metrics ..................... 57

5.3 .3 Generic Token Detection . . . . . . . . . . . . 57

5.3 .4 Alias Detection . . . . . . . . . . . . . . 58

5.3.5 Evaluation on Labeled Datasets . . . . . . . . . . . . . . . 59

5.3.6 Evaluation on Unlabeled Datasets . . . . . . . . . . 61

5.4 Discussion . . . . . . . . . . . . . . . . 62

6 Conclusions $\quad 65$

6.1 Funding Acknowledgments . . . . . . . . . . . . . . 66

$\begin{array}{lr}\text { Bibliography } & 67\end{array}$ 


\section{List of Figures}

3.1 A summary of our approach for one download portal, excluding the preparation step. . . . . . . . . . . . . . . . . 17

4.1 High level architecture. . . . . . . . . . . . . . . . 34

5.1 AVCLASS architecture. . . . . . . . . . . . . . . . 49

5.2 Running example. . . . . . . . . . . . . . . . 50

5.3 Parameter selection for generic token detection. . . . . . . . . . . 58

5.4 Parameter selection for alias detection. . . . . . . . . . . 58 


\section{List of Tables}

2.1 Related work that uses AV labels. The number of samples includes only those labeled using AV results and for classification approaches only malicious samples. . . . . . . . . . . . . . . . . .

3.1 Download portals analyzed in this work, their Alexa ranking (from October $\left.10^{\text {th }}, 2016\right)$, whether they accept software submissions through forms or PAD files, and the platforms covered (Windows, Android, MacOS, Linux). GP means it redirects to Google Play. . . . . . . . . 16

3.2 Download portals crawling results. . . . . . . . . . . . 22

3.3 Percentage of undesirable programs in each download portal. . . . . . 23

3.4 Top 10 PUP and malware families. . . . . . . . . . . . . . . . 24

3.5 Top 10 undesirable authors with more than 50 files. . . . . . . . . . 26

4.1 Installer builders detected by AVCLASS. . . . . . . . . . . . 32

4.2 Behaviors monitored during execution. . . . . . . . . . 37

4.3 Summary of installation runs . . . . . . . . . . . . . . . . . . . 43

4.4 Split of programs by installer type . . . . . . . . . . . . . 44

4.5 PUP detection on 16,547 installations $\ldots \ldots \ldots 44$

5.1 AV engines found in our datasets and their lifetime in days. . . . . . 51

5.2 Categories in the manual generic token list. . . . . . . . . . 53

5.3 Top 10 families by number of aliases. . . . . . . . . . . . 55

5.4 Datasets used in evaluation. . . . . . . . . . . . . . . . 56 
5.5 Accuracy evaluation. Full Label corresponds to using a plurality vote on all labels without normalization. Manual corresponds to running AVCLASS with manually generated generic token and alias lists. AVCLASS corresponds to running AVCLASS with automatically generated generic token and alias lists. The MalGenome* row corresponds to grouping the 6 DroidKungFu variants in MalGenome into a single family. . . . . . . . . . . . . . .

5.6 Labels for the top 5 clusters identified by AVCLASS in the Miller et al. dataset and the most common full labels on the same dataset. . . . . . 60

5.7 Clustering results on unlabeled datasets. . . . . . . . . . . . 61

5.8 Top 10 clusters on unlabeled datasets. . . . . . . . . . . . . . 62

5.9 University dataset clustering with $4,10,48$, and all AVs. . . . . . . 63

5.10 Accuracy numbers reported by prior clustering works. . . . . . . 63 


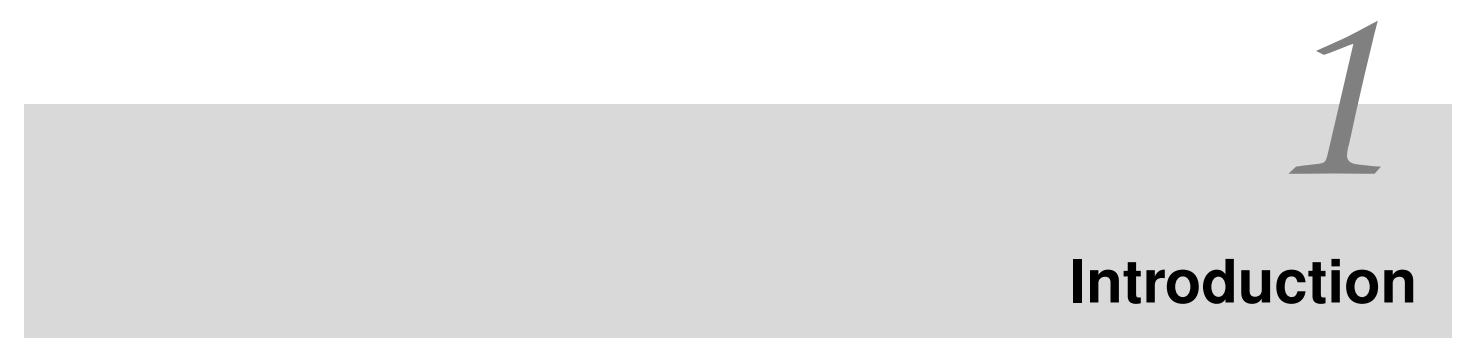

Potentially unwanted program (PUP) are a category of undesirable software, that while not outright malicious, contain behaviors considered harmful by many users. Those behaviors make them be flagged as malicious by antivirus (AV) engines. PUP subclasses include: adware designed to aggressively display advertisements, redirect search requests to advertising websites, and collect marketing data about the user; rogueware designed to mislead users into believing their hosts have security or performance issues, and to manipulate them into buying a software license that helps in fixing those issues; risktools that contain functionality that may be used with malicious intent (e.g., concealing files, hiding windows running applications, terminating active processes). Authors and publishers of PUP develop their software with behaviors that include intrusive advertising such as ad-injection, ad-replacement, pop-ups, and pop-unders; bundling programs users want with undesirable programs; setting the default installation options to accept third-party offers which may content PUP; leveraging the behavior of lazy users who want to install programs with as few clicks as possible [1]; tracking users' Internet surfing; pushing the user to buy licenses for rogueware of dubious value, e.g., registry optimizers; and abusing Windows Authenticode Code Signing [2].

PUP is prevalent, possible more than malware. During the first quarter of 2018, Kaspersky Labs detected 87M malicious software and 60\% of those were PUP such as risktools and adware [3]. Prior studies show that ad-injectors, typically browser extensions that inject advertisements into user's Web surfing, affect as many as 5\% of browsers and that malicious extensions, which evade detection in the Chrome Web Store, affect over 50 million users [4]. More recently, researchers used a database of 3.9 million hosts where they identified that $54 \%$ of the hosts were affected by PUP [5].

PUP is also profitable, its ecosystem is a lucrative strategy to monetize software, advertising, and Internet traffic [4,6]. An study conducted by the Association of National Advertisers (US) and the firm White Ops [7] has estimated the global cost of adware in 2017 to be $\$ 6.5$ billion, and the World Federation of Advertisers [8] estimates that adware will cost $\$ 50$ billion a year by 2025. Panda Security [9] estimates in 2010 that $3.5 \%$ of all computers were infected with rogueware, generating a revenue 
of more than $\$ 400$ million a year.

In response to the increasing number of PUP, security vendors and big software companies have proposed guidelines and policies [10-13] to delineate the behaviors that make a program PUP. Behaviors used to detect PUP are related to program distribution, program installation, run-time behaviors, and program uninstallation. However, those guidelines are designed for manual analysis. Automatic tools for detecting and analyzing PUP behaviors are needed.

PUP detection challenges. Currently, unwanted software detection approaches are binary, where the strict two options are malware or benign. However, the evolution of undesirable programs demands more fine grained detection approaches, because to the best of our knowledge there are no approaches which are capable of detecting PUP behaviors in order to determine whether a software is malware, PUP, or benign. Another challenge faced by PUP detection is that AV engines focus on detection of malicious software, but they do not determine automatically if the executable is PUP. This is likely due to PUP being distributed as installers. To identify a PUP program, the analysis should cover the program installation, the uninstallation of all programs installed by the installer, and the joint analysis of the installation and the uninstallations to determine whether the software transgresses the aforementioned policies.

PUP analysis challenges. An important challenge of analyzing PUP is its distribution. PUP and malware distribution use different vectors to install their ware on as many victim machines as possible. Malware uses deceitful vectors to execute or install on victim's machines such as drive-by downloads [14-16], where vulnerability exploitation distributes the malware, and performs a silent installation on the victim machine. In contrast, PUP is not installed silently because otherwise, security vendors would flag them as malware. Indeed PUP is installed with the consent of the user, who (consciously or not) approves the PUP installation on its host [5]. This PUP behavior typically happens with some installers that are distributed through download portals, i.e., websites that index, categorize, and host programs.

Other challenges for analyzing PUP and malware are associated with labeling and classification. Malicious developers rapidly evolve their products, applying polymorphic techniques to evade detection and classification. This, together with the constant production of new malicious software [3] demands approaches capable of classifying millions of malicious samples. But, classification approaches using supervised machine learning require a ground truth set of malicious samples $[17,18]$, which is not always available, creating a chicken-and-egg problem. This, in turn, makes the labels assigned by AV vendors to detected samples become the unique available reference datasets, even when they are well-known to be inconsistent [19].

In this thesis, we develop tools to detect and analyze potentially unwanted programs. More concretely we present three main contributions. First, we propose the first system to dynamically analyze and detect PUP behaviors, through evaluating the 
behaviors during installation and uninstallation phases. Second, we present a measurement study of PUP prevalence in download portals, and we expose the abusive behaviors that authors of malicious software use to distribute their applications through download portals. Third, we describe AVCLASS, an automatic labeling tool that given the AV labels for a potentially massive number of samples, outputs the most likely family for each sample.

\subsection{PUP Detection}

Programs are often distributed as installers, an auxiliary executable program responsible for performing all installation steps for the target program. Those steps may include checking if system requirements and dependencies are met, identifying and uninstalling older program versions, writing program files into a specific folder structure, adding keys into the Windows Registry, installing device drivers, generating configuration files, configuring persistent services that run automatically (e.g., by adding Windows registry keys or a cron job), downloading resources from the Internet, making the program easy to launch (e.g., by creating links or shortcuts), activating the software, checking for available newer versions, and so on. Similarly, the uninstallation process can also be complex, as it needs to revert the installation steps. An installer in most of the cases uses a GUI that presents windows that require human interaction. It may also offer to users third-party software through pay-per-install (PPI), a mechanism to monetize software distribution [5,20,21].

Current malware detector systems face difficulties to detect PUP behaviors, due to two main reasons. First, they analyze at the granularity of a single executable/process. However, when software is being installed, it may create several processes and install several programs and services. Second, because most of the PUP behaviors described in the guidelines of security vendors and software companies [10-13] are related to system modifications during the installation and uninstallation phases, but malware detector systems do not analyze these two phases together, so they cannot establish a relationship between both phases.

In this thesis, we detail what we believe is the first tool to detect PUP behaviors by performing an automatic analysis of program installation and uninstallation. We have implemented our platform as a sandbox to automatically install programs, reboot the virtual machine, and execute the uninstallation, and output two logs one of the installation and one of the uninstallation. The installation log identifies the installed programs, clustering the components that belong to the same program (e.g., files, links, tasks); the installer type that installed the program; the program uninstallation commands (if any); and the possible program names. The uninstallation log identifies how complete was the uninstallation, i.e., analyzing if all the modifications in the system during the installation have their counterparts in the uninstallation. 


\subsection{Measuring PUP and Malware Distribution in Download Portals}

Nowadays people are always connected to the Internet, using it for work, entertainment, and as a source of information. When users need to use a program they search on the Web, and the first results for the target program typically come from download portals. Most users open those links and start looking for the download link in the download portal. Then, they get the file and install it, but in some occasions instead of getting only the target program, the user ends up with one or more undesirable software in its machine. This happens when the download portal has been abused to distribute PUP or malware.

To distribute undesirable software, their authors use vectors that include drive-by download for malware [14-16], PPI services for malware and PUP [5, 21], and download portals. The abuse of download portals can be due to PUP and malware authors uploading their ware, by benign freeware authors joining as affiliate publishers of PPI services and other affiliate programs, or by malicious download portal owners. Analysts have reported that most of the programs offered in download portals are PUP [1,22-26]. Those prior works share the limitation of taking into account only the promoted applications and top downloaded programs of few download portals. Those promoted or top applications are typically the same across several download portals, and may not be representative of all programs served by a download portal.

In this thesis, we present a measurement study of the prevalence of PUP and malware in 20 download portals. We have built a platform to download and execute all programs offered by a download portal, regardless of whether they are in the list of promoted or top downloads. Then we quantify the ratio of undesirable programs in download portals, and we detail several abusive behaviors that authors of undesirable programs use to distribute their programs through download portals.

\subsection{PUP and Malware Labeling}

Labeling is the process of assigning a family name to a malware or PUP sample. The name that each AV assigns to each sample is what we call an AV label. Labeling a malicious executable as a variant of a known family is important for multiple applications such as triage, attribution, lineage and classification. New variants of a malware or PUP family constantly appear due to polymorphism used by malicious authors to avoid detection. Labeling new samples as variants of a known family allows selecting the appropriate disinfection mechanism, potentially attributing a malware to its author, and focusing the analysis on new (i.e., unlabeled) samples.

Labeling is typically done in one of three ways: the first one is manually by analysts, which is not a scalable approach; the second one is automatically by malware classification approaches using supervised machine learning (assuming the sample belongs to a family in the training set), and the third one through malware clustering 
approaches followed by a manual labeling process to assign a known family name to each cluster. Most current approaches for extracting family information from AV labels are manual and inaccurate. Oftentimes, such labeling is based on labels output by antivirus engines. While AV labels are well-known to be inconsistent [27-29], there is often no other information available, and those labels enable great scalability to perform the labeling massively. Thus, security analysts keep relying on them.

PUP and malware labeling presents some challenges. There have been attempts at reducing confusion in AV labels through naming conventions [30,31], but they have not gained much traction. Usually, AV vendors assign different labels to a sample, which produces AV vendor discrepancies. Some labeling approaches use a fixed list of $\mathrm{AV}$ engines, but this is problematic because some $\mathrm{AVs}$ can be good with some families, but poor with others. Another challenge is automation since more than one million of new malicious samples are collected daily [32], making the task of manual labeling unrealistic.

In this thesis we describe AVCLASS, an automatic labeling tool that given the AV labels for a potentially massive number of samples, outputs the most likely family names for each sample. AVCLASS implements novel automatic techniques to address 3 key challenges: normalization, removal of generic tokens, and alias detection. It offers the following seven characteristics: First, it is automatic, avoiding manual analysis limitations on the size of the input dataset. We have evaluated AVCLASS on 8.9M malicious samples. Second, it is vendor-agnostic; prior work operates on the labels of a fixed subset of 1 to $48 \mathrm{AV}$ engines. In contrast, AVCLASS operates on the labels of any available set of AV engines. Third, it performs a plurality vote on the normalized family names used by all input engines, which outputs a family name more often than a majority vote. Fourth, it can cluster samples for any platforms supported by the AV engines. We evaluate AVCLASS on Windows and Android samples. Fifth, it does not require executables. AV labels can be obtained from online services like VirusTotal [33] using the hash of the sample, even when the executable is not available. Sixth, the accuracy of AVCLASS has been evaluated on 5 publicly available malware datasets with ground truth, showing that it can achieve an F1 score of up to 93.9. And, seventh, it is reproducible: we describe it in detail and have released its source code [34]. Moreover, since we released AVCLASS in July 2016, it has became a popular tool, and has been used by multiple research groups [35-46]. Among these works, Lever et al. [47], further demonstrated the scalability of AVCLASS by applying it 23.9M samples.

\subsection{Thesis Contributions}

The main contributions of this thesis are:

- We perform a measurement study of abuse in download portals. We collected a dataset of $191 \mathrm{~K}$ Windows freeware installers from 20 download portals. We measure an overall ratio of PUP and malware between $8 \%$ (conservative estimate) and $26 \%$ (lax estimate). In 18 of the 20 download portals examined the 
amount of PUP and malware is below 9\%. But, we also find two download portals exclusively used to distribute PPI downloaders. We detail different abusive behaviors that authors of undesirable programs use to distribute their programs through download portals such as uploading the same file as different programs, using external links to bypass security checks, and impersonating benign popular authors.

- We build a platform to perform dynamic behavioral detection that focuses on automatically detecting PUP. The platform analyzes an input installer, performs the installation, identifies the list of installed programs, whether each program has an uninstaller, performs the uninstallations, checks for PUP behaviors in both installation and uninstallations, and determines if all programs installed were completely uninstalled. We have evaluated our platform using two datasets: $16 \mathrm{~K}$ freeware installers samples collected from download portals, and a small dataset of 50 benign samples.

- We present AVCLASS, an automatic labeling tool that given the AV labels for a, potentially massive, number of samples outputs the most likely family names for each sample, ranking each candidate family name by the number of AV engines assigning it to the sample. We have evaluated AVCLASS on 10 datasets comprising 8.9 M samples. AVCLASS's clustering achieves F1 measures up to 93.9 on labeled datasets and clusters are labeled with fine-grained family names commonly used by the AV vendors.

\subsection{Thesis Outline}

The remainder of this thesis is organized as follows. In Chapter 2, we present the related work on potentially unwanted programs, installation and uninstallation of malicious executables, and labeling. The thesis contributions are presented in Chapters 3 to 5. In Chapter 3, we present a measurement study of prevalence of PUP and malware in download portals. Chapter 4 details a tool to analyze and detect PUP behaviors of executables by performing an automatic analysis of the installation and uninstallation executions. Chapter 5 describes AVCLASS, a tool for automatically labeling malicious samples. Finally, in Chapter 6 we conclude and discuss areas of future work. 


\section{Related Work}

To analyze PUP thoroughly this thesis focuses on three main PUP aspects: PUP distribution through download portals, PUP detection through installation and uninstallation behaviors, and PUP and malware labeling. In this chapter we present related work divided into these categories.

\subsection{PUP Distribution Through Download Portals}

Download portals. Security vendors have analyzed the top downloads of download portals and concluded that they are bloated with PUP [23, 25, 48]. In concurrent and independent work, Geniola et al. [1] collect 800 installers of promoted applications from 8 download portals. They execute them in a sandbox and find that $1.3 \%$ of those installers drop well-known PUP to the system and 10\% install a browser or a browser extension. One main goal of this work is measuring the amount of abuse in download portals, i.e., the percentage of PUP and malware. The main limitation of prior works towards that goal is that the analyze only the top downloaded programs or the promoted applications, which may not be representative of all distributed programs. In contrast, we have downloaded all the Windows programs offered by 20 download portals. We have collected 75,615 unique executables, almost two orders of magnitude more than prior works. Our results show an overall ratio of PUP and malware between $8 \%$ and $26 \%$, significantly higher than the $1.3 \%$ reported by Geniola et al. Our analysis also identifies two download portals, part of a PPI distribution service, which serve $100 \%$ PUP. Finally, we have identified abusive behaviors PUP authors employ to distribute their programs through download portals.

PUP. Early work on PUP focuses on what constitutes PUP [49-51] and its deceptive methods [52-54]. Research on PUP has recently revived with a number of papers examining PUP prevalence and its distribution through commercial PPI services. Thomas et al. [4] measured that ad-injectors, a type of PUP that modifies browser sessions to inject advertisements, affect $5 \%$ of unique daily IP addresses accessing Google. Kotzias 
et al. [2] studied abuse in Windows Authenticode by analyzing 356K samples from malware feeds. They found that PUP has quickly increased in so-called malware feeds since 2010, that the vast majority of properly signed samples are PUP, and that PUP publishers use high file and certificate polymorphism to evade security tools and CA defenses such as identity validation and revocation. In a separate work, Kotzias et al. [5] used AV telemetry of 3.9 M real hosts for analyzing PUP prevalence and its distribution through commercial PPI services. They found PUP installed in 54\% of the hosts and identified 24 commercial PPI services that distribute over a quarter of all the PUP in their 2013-14 dataset. They also determined that commercial PPI services used to distribute PUP are disjoint from underground PPI services used to distribute malware [55]. In simultaneous and independent work, Thomas et al. [21] analyzed the advertiser software distributed to US hosts by 4 commercial PPI services. They used SafeBrowsing data to measure that PPI services drive over 60 million download events every week in the second half of 2015, nearly three times that of malware. Nelms et al. [56] analyzed web-based advertisements that use social engineering to deceive users to download PUP. They found that most programs distributed this way are bundles of free software with PUP. This work differs from the above in that it analyzes PUP prevalence in download portals.

\subsection{PUP Detection Through Installation and Uninstal- lation Behaviors}

Dynamic malware analysis systems Many works have proposed sandbox systems for dynamic malware analysis [57-62]. Those may use in-guest tracing of Windows API calls [58,60], emulation [57,62], hardware-supported virtualization [61], and bare machines [59]. Those systems are designed to analyze malware behaviors. They analyze each execution in isolation, regardless whether it is an installer or an standalone executable. However, PUP analysis requires specific systems that handle installers and uninstallers, perform GUI exploration, detect PUP-specific behaviors, identify the list of installed programs, and can jointly analyze the installation and uninstallation runs. Our platform is specifically designed to address these issues.

Uninstallation. Prior work has proposed disinfection mechanisms to revert malware's infections. Passerini et al. [63] proposed an automated testing methodology to evaluate the completeness of remediation procedures in commercial malware detectors, where they observed that only about $80 \%$ of the untrusted executables dropped by malicious programs on infected systems are properly removed by malware detectors. Paleari et al. [64] presents an architecture to automatically generate remediation procedures given binary malware samples. They evaluate their approach on a dataset of 200 malicious samples, finding that their technique was able to remediate $98 \%$ of the harmful effects of the malware infection. Hsu et al. [65] proposed a framework 
for automatically removing malware from and repairing its damage to a system. They detect malware's modification and compare them with two commercial anti-malware software.

Table 2.1: Related work that uses AV labels. The number of samples includes only those labeled using AV results and for classification approaches only malicious samples.

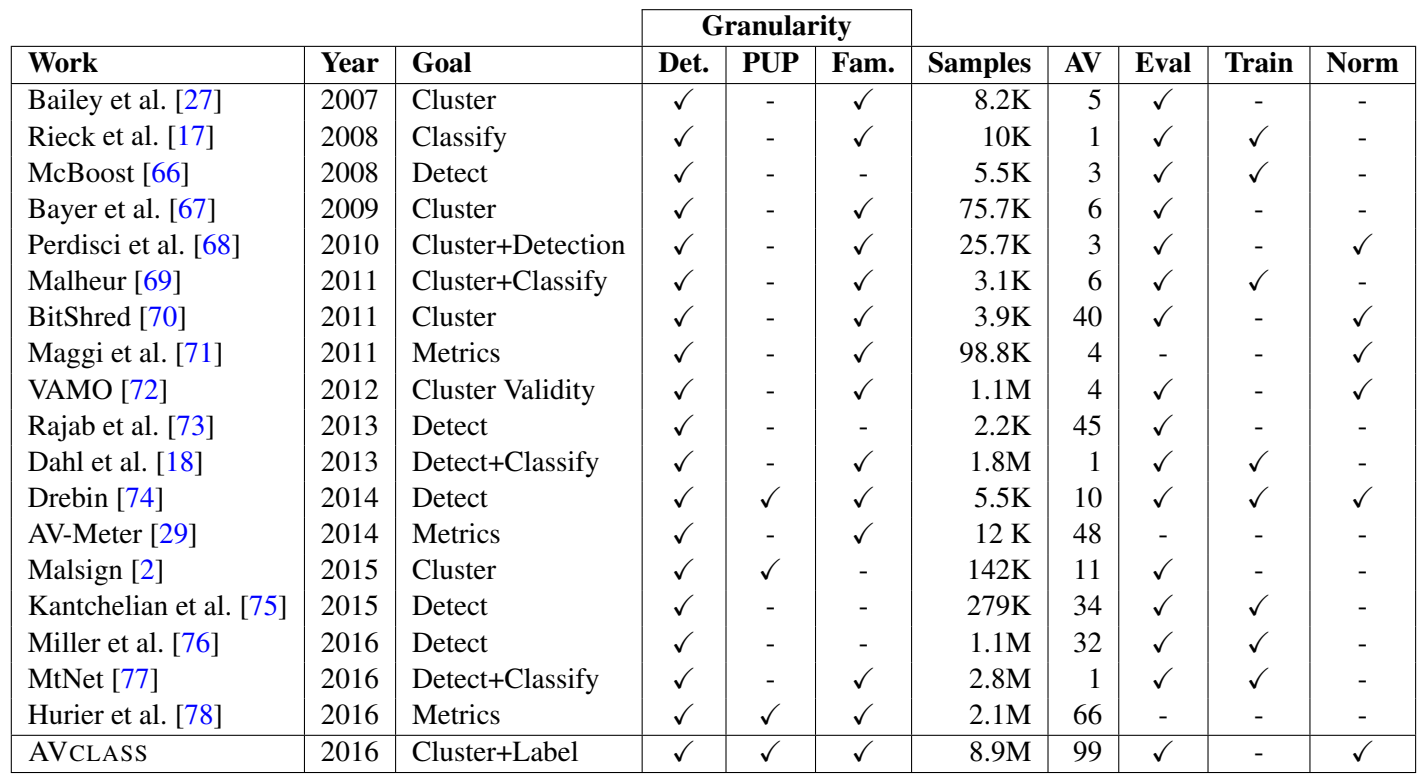

\subsection{PUP and Malware Labeling}

Table 2.1 summarizes relevant works that have used AV labels. For each work, it first presents the year of publication and the goal of the work, which can be malware detection, clustering, classification, combinations of those, cluster validity evaluation, as well as the development of metrics to evaluate AV labels. Then, it describes the granularity of the information extracted from the AV labels, which can be Boolean detection (i.e., existence or absence of the label), coarse-grained classification in particular if a sample is a potentially unwanted program (PUP) or malware, and extracting the family name. Next, it shows the number of labeled samples used in the evaluation and the number of vendors those labels come from. For supervised approaches, the samples column includes only malicious samples in the training set. As far as we know the largest dataset used previously for malware clustering or classification is by Huang and Stokes [77], which comprises 6.5 M samples: 2.8 M malicious and 3.7 M benign. In contrast, we evaluate AVCLASS on $8.9 \mathrm{M}$ malicious samples, making it the largest so far. The next two columns capture whether the AV labels are used to evaluate the results and for training machine learning supervised approaches. Finally, the last col- 
umn captures if normalization is applied to the AV labels $(\checkmark)$, or alternatively the full label is used (-).

Most works consider a sample malicious if at least a threshold of AV engines detects it (i.e., returns a label) and weigh each AV engine equally in the decision. There is no agreement in the threshold value, which can be a single AV engine [72,79], two [74], four $[2,76]$, or twenty [70]. Some works evaluate different thresholds [29, 80] showing that small threshold increases, quickly reduce the number of samples considered malicious. Recently, Kantchelian et al. [75] propose techniques for weighing AV engines differently. However, they assume AV labels are independent of each other, despite prior work having found clusters of AV engines that copy the label of a leader [29], which we also observe. In addition, an AV engine with poor overall labels may have highly accurate signatures for some malware families. In our work we adjust the influence of AV engines that copy labels and then weigh remaining labels equally.

Other works show that AVs may change their signatures over time, refining their labels [75, 81]. Recently, Miller et al. [76] argue that detection systems should be trained not with the labels available at evaluation time (e.g., latest VT reports), but with the labels available at training time. Otherwise, detection rate can be inflated by almost 20 percentage points. However, for evaluating clustering results, it makes sense to use the most recent (and refined) labels. Miller et al. [76] evaluate a semi-supervised scenario and show that having a limited number of manual labels can vastly improve the detection capabilities.

AV label inconsistencies. Prior work has identified the problem of different AV engines disagreeing on labels for the same sample [27-29,71]. While such discrepancies are problematic, security analysts keep coming back to AV labels for ground truth. Thus, we believe the key question is how to automatically extract as much family information as possible from those labels and to quantitatively evaluate the resulting reference dataset. We propose an automatic labeling approach that addresses the most important causes for discrepancies, namely different naming schemes, generic tokens, and aliases. Li et al. [82] analyze the use of a reference clustering extracted from AV labels to evaluate clustering results. They argue that using only a subset of samples, for which the majority of AV engines agrees, biases the evaluation towards easy-tocluster samples. AVCLASS automatically extracts the most likely family names for a sample (even if no majority agrees on it), helping to address this concern by enlarging the reference dataset. They also observe that imbalance distributions of samples in the input dataset may cluster the clustering results towards those families. Mohaisen and Alrawi [29] propose metrics for evaluating AV detections and their labels. They show how multiple AVs are complementary in their detection and also that engines with better detection rate do not necessarily have higher correctness in their family names. Recently, Hurier et al. [78] propose further metrics to evaluate ground truth datasets built using AV labels. One limitation of proposed metrics is that they operate on the full AV labels without normalization. Most related to our work is VAMO [72], which proposes an automated approach for evaluating clustering results. VAMO normalizes 
the labels of $4 \mathrm{AV}$ vendors to build an AV graph (introduced in [68]) that captures the fraction of samples where labels, possibly from different engines, appear together. Our alias detection approach is related, although VAMO does not output aliases as AVCLASS does. Furthermore,VAMO finds the set of reference clusters from the AV labels that best agrees with a third-party clustering, while AVCLASS labels samples without requiring third-party clustering results.

Naming conventions. There have been attempts at reducing confusion in malware labels through naming conventions, but they have not gained much traction. A pioneering effort was the 1991 CARO Virus Naming Convention [30]. More recently, the Common Malware Enumeration (CME) Initiative [31] provides unique identifiers for referencing the same malware across different names. Also related is the Malware Attribute Enumeration and Characterization (MAEC) lware Attribute Enumeration and Characterization (MAEC) language for encoding detailed information about malware [83]. 


\section{PUP Distribution Through Download Portals}

\subsection{Introduction}

Freeware is proprietary software that can be used without monetary cost. Freeware is distributed in binary form and should not be confused with open-source software that is also free but provides access to its source code. A related model is shareware where the software is initially free to use, but users are expected to pay to continue using it. In contrast, freeware is free to use for unlimited time. While freeware can be used free of charge, authors may want to cover their development costs and benefit from their freeware. This can be achieved through freemium models where the user pays for advanced functionality, voluntary user donations, advertisements, and by offering third-party software through commercial pay-per-install (PPI) services [5,21]. For example, Skype uses a freemium model where users pay for calling phone numbers and Sun's Java offers users to also install the Yahoo toolbar.

A popular vector for distributing freeware are download portals, which are websites that index, categorize, and host programs. Download portals such as cnet [84], softonic [85], or tucows [86] are a meeting point for freeware authors that want to advertise their programs and for users looking for a specific program or functionality. Users can leverage download portals for searching for popular freeware in a category (e.g., video software, security tools, Windows themes), browsing through the program metadata (e.g., version, author, platform), reading program reviews, and eventually downloading the chosen programs. Download portals enable freeware authors to distribute their programs, increasing their user base. Using download portals, the freeware author can save on advertisements costs required to let users know about the freeware's existence. Authors on a low budget can also avoid setting up a webpage for the freeware. The download portals invest in advertising and have a motivation to rank highly on search engine results to attract users that can be monetized through advertisements and PPI schemes.

Download portals can be abused as a distribution vector for potentially unwanted programs (PUP) and malware. PUP are a category of undesirable software, that while 
not outright malicious like malware, contain behaviors considered harmful by many users. While the boundary between PUP and malware is sometimes blurry, prior work has tried to delineate what constitutes PUP [49-51] and companies such as Google [11], Microsoft [10], and MalwareBytes [12] have policies for defining what behaviors make a program PUP. Two types of programs that are widely considered PUP are adware that aggressively pushes advertisements and rogueware that scares users into buying a software license, despite its limited functionality.

Download portals may be used to distribute PUP and malware in three ways. First, download portals can be abused by PUP and malware authors to distribute their programs, by uploading their undesirable software and disguising it as potentially useful freeware. Second, authors of benign freeware may become affiliate publishers of commercial PPI services, bundling their freeware with a PPI downloader and uploading the bundle to a download portal. When installing the bundle, users will be offered third-party advertiser programs, which may be PUP. In fact, prior work has measured that at least $25 \%$ of PUP is distributed through 24 commercial PPI services [5]. Third, download portal owners may be untrustworthy and use their download portals to purposefully distribute undesirable software to visitors.

While several blog posts point to download portals being bloated with PUP [23, 25, 48], their conclusions are based on ad-hoc measurements performed on the top downloaded programs of a few download portals. In this work, we perform a systematic study of abuse in download portals. We build a platform to crawl download portals. We use our platform to download all Windows freeware offered by 20 download portals. This enables examining PUP and malware prevalence beyond that of the most popular downloads. Our crawling downloads $191 \mathrm{~K}$ programs from the 20 download portals, which correspond to $157 \mathrm{~K}$ unique files with a cumulative size of $2.5 \mathrm{~TB}$. We analyze the collected freeware to identify PUP and malware and execute the programs in a sandbox to analyze what modifications they perform to the system, e.g., changing a browser's homepage and installing browser modifications. Our analysis addresses the following three main questions:

- What percentage of programs in download portals are PUP and malware? We use two policies to quantify undesirable (i.e., PUP or malware) programs in download portals. Our conservative policy identifies as undesirable any program flagged by more than $3 \mathrm{AV}$ engines, while our lax policy identifies as undesirable any program flagged by at least one AV engine. We measure an overall ratio of undesirable programs across all download portals analyzed ranging between $8 \%$ (conservative) and 26\% (lax). Among the undesirable programs PUP (76\%) dominates malware $(24 \%)$. For 18 of the 20 download portals examined, the amount of PUP and malware is moderate ranging between $8.5 \%$ and a low as $0.2 \%$. These ratios are significantly lower than those reported in prior works that only examine the top downloads $[23,25,48]$. We believe our measurements are more accurate since we examine all programs indexed by a download portal.

- Are there download portals that are clearly abusive? We identify two down- 
load portals, run by the same company, which serve $100 \%$ PUP. Those download portals are exclusively used to distribute a PPI downloader. Regardless what program the user chooses to download, he is always provided with a customized PPI downloader. We provide a detailed analysis of the operation leveraging those two download portals and identify another 12 similar download portals from the same owners.

- How are download portals abused? Our analysis uncovers different abusive behaviors that authors of undesirable programs employ to distribute their programs through download portals. We observe authors uploading the same file as different programs in the same download portal, as well as across multiple download portals. We identify some authors using external links to bypass security checks by download portals. We show that the failure to identify repetitive abusers is widespread across download portals, rather than limited to a few careless download portals. Finally, we observe impersonation of benign popular authors by other authors that want to leverage their reputation, e.g., to disguise their undesirable programs as innocuous.

\subsection{Download Portals}

Download portals index large amounts of programs from different authors. To enable users finding the program they are interested in, or a program that matches a specific functionality, programs are typically grouped into categories and indexed using keywords. Download portals have existed for at least 20 years with popular download portals such as cnet and softonic being launched in 1996 and 1997, respectively. The download portals may host the programs themselves, i.e., the file is downloaded from domains owned by the download portal; may link to the author's webpage where the program is hosted; or may provide both types of downloads.

Most download portals accept submissions from software developers through forms where a developer specifies information about its software such as program name, version, description, and program's URL. Each download portal requires different information from the developers. Some download portals also support submissions through the Portable Application Description (PAD) standard, an XML schema introduced in 1998 by the Association of Software Publishers (ASP) to standardize software metadata [87].

Download portals face challenges in determining that a submitted program matches its description and that it is uploaded by its real author. Some download portals may take steps towards reducing abuse, e.g., analyzing submitted files using online services such as VirusTotal (VT) [33]. Recently, some download portals like filehippo and softonic, have stopped accepting submissions by developers. These download portals analyze the freeware ecosystem themselves to select new programs to add. 
Pay-per-install. A popular software monetization mechanism are pay-per-install (PPI) agreements where an advertiser, i.e., a software publisher interested in distributing its program to users, pays a third-party to help with the distribution. PPI agreements can be bilateral between two software publishers, e.g., Oracle distributing the Ask toolbar with its Java platform [88]. They can also take the form of commercial PPI services that connect multiple advertisers interested in distributing their programs with multiple affiliate publishers willing to offer those advertised programs to users that install the affiliate's program [5,21]. Affiliate publishers are often freeware authors that own programs that users want to install. They bundle their freeware with a PPI downloader and distribute the bundle, e.g., by uploading the bundle to download portals, in exchange for a commission paid for each installation. When a user installs the bundled freeware, the PPI downloader offers to the user the advertised programs. If the user installs an advertised program, the advertiser pays the PPI service for the installation and the affiliate publisher receives a commission. Some download portals such as cnet run their own commercial PPI service to supplement their advertisement income. Freeware authors uploading their programs to the download portal are invited to join as publishers of the download portal's PPI service to monetize their programs.

An alternative PPI model is for software publishers to directly recruit affiliates to distribute their software without using a PPI service. Some large PUP publishers have affiliate programs such as Mindspark, Babylon, Systweak, and Spigot [5]. Freeware authors can sign up as affiliates of such programs, obtain a bundle and distribute it, e.g., by uploading to download portals.

Installers. Most programs need to be installed before they can be executed. The installation process may, among others, check if system requirements and dependencies are met, setup program files into a specific folder structure, configure services that run automatically, download resources from the Internet, and make the program easy to launch (e.g., adding shortcuts and entries to the start menu). To ease installation, programs are often distributed as installers, i.e., auxiliary programs (e.g., setup.exe) responsible for installing a target program. Most files downloaded from download portals correspond to installers.

Analyzed download portals. We analyze 20 download portals that offer Windows programs. The selected portals may offer programs for other platforms as well, but we crawl only the Windows programs they offer. We have selected download portals that target different geographic locations and of different popularity (according to their Alexa ranking [106]) because there may be differences in behaviors between those types, e.g., how they vet publishers leading to different amounts of abuse. Table 3.1 shows the list of 20 download portals analyzed in this work. For each download portal it shows the abbreviated name we use to refer to the download portal, its URL, the Alexa ranking, whether the download portal accepts software submissions using forms and PAD files, and the platforms for which it indexes programs. Download portals that 
Table 3.1: Download portals analyzed in this work, their Alexa ranking (from October $\left.10^{\text {th }}, 2016\right)$, whether they accept software submissions through forms or PAD files, and the platforms covered (Windows, Android, MacOS, Linux). GP means it redirects to Google Play.

\begin{tabular}{|l|r|cc|cccc|}
\cline { 3 - 8 } \multicolumn{2}{c|}{} & \multicolumn{2}{c|}{ Submission } & \multicolumn{5}{c|}{ Platforms } \\
\hline Portal Name & Alexa & Form & PAD & Win. & And. & Mac & Lin. \\
cnet [84] [89] & 155 & $\checkmark$ & - & $\checkmark$ & $\checkmark$ & $\checkmark$ & $\checkmark$ \\
softonic [85] & 164 & $\checkmark$ & $\checkmark$ & $\checkmark$ & $\checkmark$ & $\checkmark$ & - \\
filehippo [90] & 200 & - & - & $\checkmark$ & GP & $\checkmark$ & - \\
softpedia [91] & 615 & - & - & $\checkmark$ & - & - & - \\
soft112 [92] & 1,589 & $\checkmark$ & $\checkmark$ & $\checkmark$ & $\checkmark$ & - & - \\
majorgeeks [93] & 4,672 & - & $\checkmark$ & $\checkmark$ & GP & $\checkmark$ & $\checkmark$ \\
soft32 [94] & 6,206 & - & - & $\checkmark$ & GP & - & - \\
eazel [95] & 6,640 & $\checkmark$ & $\checkmark$ & $\checkmark$ & $\checkmark$ & $\checkmark$ & - \\
fileforum [96] & 8,760 & - & - & $\checkmark$ & $\checkmark$ & $\checkmark$ & - \\
filehorse [97] & 9,449 & $\checkmark$ & - & $\checkmark$ & - & $\checkmark$ & $\checkmark$ \\
portalprogramas [98] & 9,980 & - & - & $\checkmark$ & - & $\checkmark$ & - \\
freewarefiles [99] & 12,171 & $\checkmark$ & - & $\checkmark$ & $\checkmark$ & $\checkmark$ & - \\
tucows [86] & 13,556 & $\checkmark$ & - & $\checkmark$ & - & - & - \\
snapfiles [100] & 25,084 & $\checkmark$ & $\checkmark$ & $\checkmark$ & - & $\checkmark$ & $\checkmark$ \\
filecluster [101] & 33,545 & $\checkmark$ & - & $\checkmark$ & - & - & - \\
descargarmp3 [102] & 56,379 & $\checkmark$ & $\checkmark$ & $\checkmark$ & - & - & - \\
download3000 [103] & 104,352 & - & - & $\checkmark$ & GP & - & - \\
fileguru [104] & 230,115 & $\checkmark$ & - & $\checkmark$ & - & - & - \\
geardownload [105] & 308,929 & $\checkmark$ & $\checkmark$ & $\checkmark$ & - & - & - \\
\hline
\end{tabular}

do not accept submissions through forms nor PAD may accept them through email or not accept submissions at all.

\subsection{Approach}

Our approach processes one download portal at a time and comprises four steps: preparation, crawling, file processing, and execution. During preparation, an analyst manually generates one portal metadata file for the download portal, which contains all the needed information to crawl the download portal. The crawling, file processing, and execution steps are automated and illustrated in Figure 3.1. The crawling takes as input a portal metadata file, and automatically navigates a Selenium-based crawler [107] to download all the programs the download portal offers. It outputs the downloaded files and saves all information about the crawling into a central database. The file processing extracts information statically from the downloaded files (e.g., filename, filetype), collects the file report from VirusTotal [33], extracts executable files from archives, 


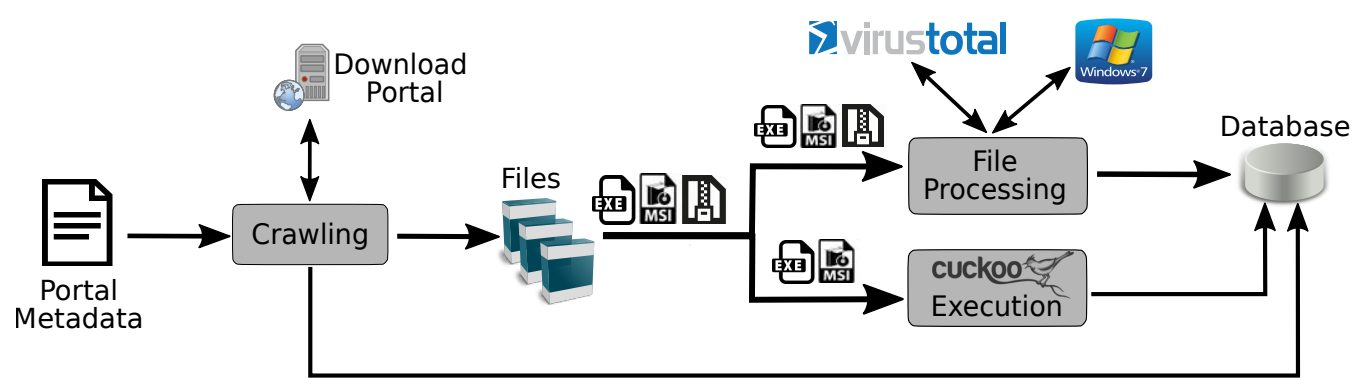

Figure 3.1: A summary of our approach for one download portal, excluding the preparation step.

and checks the Authenticode digital signature (if signed). The execution takes as input the programs, runs them in a sandbox, generates an execution report, and saves the report data in the central database. Each of these four steps is detailed next in its own subsection.

\subsubsection{Preparation}

Download portals share the goal of enabling users to find the software they are interested in. This creates similarities in their structure and design. However, each download portal is different. For example, download portals differ in their layout and the information they collect about the programs. This makes it challenging to crawl a large number of download portals to analyze the software they index. To address this issue, at the beginning of this project we studied multiple download portals to identify a common abstraction that worked for all of them and enables adding a new download portal to be crawled with very limited effort.

All the download portals we have examined share a basic structure in which every program in the download portal has its own program page, which presents the data that the download portal has collected on the program. The program page may contain, among others, the program name, version, description, license, platform, language, size, author, number of downloads, reviews, date of publication, date of last update, screenshots, previous versions, and download links. The specific program attributes in the program page vary across download portals. Different programs within the same download portal may have different program attributes, e.g., if an upload form has optional fields that some authors fill and others do not. However, we have been able to identify a subset of 6 program attributes that are available for all programs in all download portals analyzed: name, version, platform, size, author, and download link.

The output of the preparation step is a portal metadata file that has all the information needed for the crawling. Specifically, the portal metadata file contains three parts: how to list all the program pages, how to identify the 6 program attributes from the program page, and how to download the program file from the download link or button in the program page. We describe them next. 
Listing the program pages. We can classify download portals into two classes regarding how to list all program pages in the download portal. Class 1 download portals allows us to list all programs directly and Class 2 download portals allows us to list all programs in each category. Class 1 download portals may offer a direct link to list all programs or may enable searching with wildcard identifiers through which we can list all programs. For class 1 download portals, we can extract a URL template such as http://portal.com/software/?page=X where $X$ is a positive integer that can be monotonically increased to iterate over the search results. Every page in this iteration contains, among other content, a list of program entries, each with a program $U R L$ that points to the program page. To identify the program URLs inside the search results pages we use the path in the page's DOM. For Class 2 download portals we need to first obtain the list of all program categories. Then, we can define a URL template such as http://portal.com/<category $>/$ ?page $=\mathrm{X}$ and we can iterate over the search results of each category by monotonically increasing $X$ and extracting the program URLs similar to Category 1 download portals. The analyst provides in the portal metadata file: an URL template for Class 1 and Class 2 download portals, and a list of all software categories for Class 2 download portals.

Identifying the program attributes. To identify the program attributes in the program page (name, version, platform, size, author, and download link), the analyst provides in the portal metadata file a DOM path for each program attribute, which uniquely identifies the position of a page element that contains the attribute.

Downloading the program files. The program page always contains an element to download the program, e.g., a download link or a download button. But, that element may not link directly to the file to download (e.g., executable or archive). Instead it may open a download page with some advertisements and one or more download links, e.g., from different mirrors. We call download URL to the URL from where our crawler downloads the program file, i.e., the URL that points to the program file. The analyst provides in the portal metadata file the sequence of clicks, i.e., DOM paths to buttons to be clicked, starting with the click on the download element in the program page, that the crawler needs to perform to arrive and click on the download URL of the latest program version.

We developed the process above iteratively. Once we converged on this processing, generating a portal metadata file for a new download portal took us 2-3 hours.

\subsubsection{Crawling}

The crawling takes as input the portal metadata file. It outputs the program files downloaded from the portal and saves into a central database the crawling information such as timestamps, URLs visited, and program attributes collected from the download portal. We use a crawler based on Selenium WebDriver [107] with Mozilla Firefox. The 
crawler follows the instructions in the portal metadata file to list all program pages. For each program page, it identifies the program attributes using the DOM paths in the portal metadata file and stores the attributes. If the program is a Windows freeware, it clicks on the download link, and follows the instructions in the portal metadata file to locate the download URL.

Once the download URL is clicked, an attempt to download the program file is performed. If the download has not started after 30 seconds, the crawler tries again. A file download may fail for the following five reasons: (1) the download link is broken; (2) the download has not completed in 5 minutes, a timeout we chose to limit the maximum size of a downloaded file. This timeout provides significant storage savings and only affects files 200MB-2GB depending on the bandwidth of the download portal (even with this limit the downloaded programs use 2.5TB disk storage); (3) any web page has not finished loading within 30 seconds; (4) the download link redirects our crawler to an external web page and does not point directly to a program file, i.e., the user is expected to locate the right download link in the publisher's webpage; (5) the download portal refreshes the webpage with which our crawler is interacting, e.g., to change an advertisement.

After each download attempt, whether successful or not, the crawler outputs a tuple with: timestamp, download portal identifier, error code, program URL, download URL, 4 program attributes from the program page (program name, size, version, and author), and a file identifier if the download was successful.

We are interested in whether the download portals host the downloaded programs onsite or simply redirect users to the publisher's webpage. For this, we manually build a mapping, using Whois and DNS information, of which domains in the download URLs belong to each download portal. For example, cnet uses two effective secondlevel domains (ESDLs) for hosting programs: cnet.com and downloadnow.com.

\subsubsection{File Processing}

The file processing step statically analyzes the files and saves all information in the central database. It first processes each downloaded file to obtain: MD5, SHA1, SHA256, size on disk, filename, and filetype. Then, it attempts to decompress archives to extract any executables inside. Note that a downloaded archive may contain other archives, so this is a recursive process, which we detail in Section 3.4.1. Next, the file hash of each executable, directly downloaded or extracted from an archive, is used to query VirusTotal [33] (VT), an online service that examines user-submitted files with a large number of security tools. At the time of writing this manuscript, VT analyzes submitted files using $70 \mathrm{AV}$ engines including all major AV vendors ${ }^{1}$. Engines are frequently updated and the list of engines evolves over time. If the file is known to VT, a report is downloaded that contains, among others, the number of AV engines that detect the file, the timestamp when the file was first submitted, and file metadata. We submit to

\footnotetext{
${ }^{1}$ https://www.virustotal.com/en/about/credits/
} 
VT all files downloaded from the download portals that are smaller than 30MB. This threshold is due to the VT API, which has a limit of 32MB. We reduced the limit to $30 \mathrm{MB}$ because we observed errors with files near the limit.

We use two maliciousness policies: conservative and lax. The conservative policy is to consider a program undesirable (i.e., PUP or malware) if it detected by more than $3 \mathrm{AV}$ engines in the VT report. This policy is designed to minimize false positives due to a few AVs committing an error in the detection. The lax policy considers undesirable any program detected by at least one AV engine. We use the lax policy as an upper bound. For programs distributed as archives, we consider them undesirable if the archive itself, or any file inside the archive, satisfies the policy.

To classify an undesirable executable as either malware or PUP, and to determine its family, we use AVClass [108], a malware labeling tool. AVClass takes as input the AV labels in a VT report; removes noise from AV labels by addressing label normalization, generic token detection, and alias detection; and outputs for each sample whether it is PUP or malware, its most likely family name, and a confidence factor based on the agreement across AV engines.

The final file processing step is to analyze the signed executables. Code signing is a technique that embeds a digital signature in an executable, which enables verifying the program's integrity and authenticating its publisher. Prior work has shown that properly signed executables detected by AVs are predominantly PUP, since it is challenging for malware to obtain a valid code signing certificate from a CA [2]. The file processing component validates the Authenticode signature in executable files. For this, it uploads all executables to a Windows VM and uses the Microsoft-provided validation tool to check if the executable is signed and whether the signature validates using different policies (e.g., default and kernel driver). Signed executables are further processed by our own code to retrieve the X.509 leaf certificate and extract, among others: Subject CN, Issuer CN, PEM and DER hashes, validity period, signing hash, digital signature algorithm, signed file hash (called Authentihash), and public key. For executables that are signed and whose signature validates, we can confidently identify the publisher's identity.

\subsubsection{Execution}

We run downloaded executables in the Cuckoo Sandbox [60]. Cuckoo receives an executable, assigns it to a VM for execution, and generates a behavioral report for the execution. We have implemented a few extensions to Cuckoo to better fit our needs. These include adding some anti-anti-evasion techniques to harden the sandbox [109, 110], extending the GUI exploration, and adding signatures for specific events that we want to be notified about. These modifications are detailed below.

The vast majority of executables downloaded from the download portals correspond to installers (e.g., firefox_setup.exe) that will install the real program binaries on the end host (e.g., firefox.exe) upon execution. Such installers are typically GUI-based and require user interaction to complete the installation. Cuckoo provides functional- 
ity to identify buttons in windows launched during the execution, and to automatically click buttons labeled with keywords such as "Next" or "Confirm" simulating the default user behavior of accepting all windows to quickly install the program. However, the list of keywords used to identify those buttons is pretty small and limited to English. Thus, we extended the keyword list and translated the keywords into popular languages such as German and Spanish.

We also extended the signatures module of Cuckoo, which enables defining signatures for events of interest that Cuckoo can directly report. This module provides performance improvements to identify those events. For example, we could scan the list of registry modifications provided by Cuckoo to see if a specific key that stores Internet Explorer's homepage has been modified. But, it is significantly more efficient to build a signature for that registry key and let Cuckoo automatically report its modification. Our signatures include events such as whether browser extensions have been installed, and whether some browser settings have been altered.

We configure Cuckoo to use 30 VirtualBox VMs on a single host running Ubuntu 16.04 LTS. Each VM is configured with $1 \mathrm{~GB}$ of RAM and $20 \mathrm{~GB}$ hard disk. The VMs run Windows 7, which is still the most popular OS [111]. Our VM image has a large number of popular programs installed such as Internet Explorer, Chrome, Firefox, Opera, Java, Adobe Reader, Adobe Flash player, and the .NET framework. This is done so that we can observe modifications that the executed programs may perform on those programs.

\subsection{Evaluation}

This section evaluates our approach. Section 3.4.1 presents the results of crawling the download portals, Section 3.4.2 examines the prevalence of undesirable programs in download portals, Section 3.4.3 compares the PUP, malware, and benign programs behaviors, Section 3.4.4 summarizes the execution results, and Section 3.4.5 provides a case study on download portals associated with PPI services.

\subsubsection{Download Portals Crawling Statistics}

In this section, we present some general statistics on the crawling. We analyze the security aspects such as prevalence of undesirable programs and abusive behaviors in the next sections.

Table 3.2 summarizes the crawling results. For each download portal it presents: the date when it was crawled (all dates from 2016), the number of Windows programs offered, the number of successfully downloaded programs, the number of unique downloaded files by hash, the size of the downloaded files (in GB), the split of the unique files by type (EXE, ZIP, RAR, MSI, other), and the percentage of unique files hosted onsite (i.e., on domains that belong to the download portal). 
Table 3.2: Download portals crawling results.

\begin{tabular}{|l|l|r|r|r|r|r|r|r|r|r|r|}
\multicolumn{2}{c|}{} & \multicolumn{9}{c|}{ Programs } & \multicolumn{4}{c|}{ File type } & Hosting \\
\hline Portal & Date & Offered & Downl. & Unique & Size (GB) & EXE & ZIP & RAR & MSI & Other & Onsite \\
\hline uptodown & $06 / 14$ & 8,115 & 7,071 & 7,066 & 227.8 & 4,882 & 1,747 & 166 & 161 & 110 & $99.8 \%$ \\
cnet & $06 / 26$ & 6,814 & 5,220 & 5,161 & 67.7 & 3,432 & 1,350 & 0 & 0 & 379 & $100.0 \%$ \\
softonic & $11 / 05$ & 23,737 & 14,575 & 14,487 & 225.3 & 8,139 & 5,075 & 292 & 342 & 639 & $98.3 \%$ \\
filehippo & $06 / 15$ & 1,274 & 1,167 & 1,163 & 38.8 & 973 & 125 & 2 & 0 & 63 & $100.0 \%$ \\
softpedia & $09 / 09$ & 69,738 & 48,747 & 48,247 & 386.0 & 20,438 & 21,855 & 1,226 & 170 & 4,558 & $39.3 \%$ \\
soft112 & $10 / 11$ & 107,642 & 44,110 & 43,078 & 287.7 & 8,908 & 24,958 & 2,457 & 838 & 5,917 & $0.0 \%$ \\
majorgeeks & $06 / 25$ & 4,712 & 4,227 & 4,223 & 63.9 & 2,498 & 1,574 & 0 & 19 & 132 & $14.6 \%$ \\
soft32 & $09 / 02$ & 8,563 & 698 & 671 & 14.5 & 345 & 287 & 3 & 3 & 33 & $99.7 \%$ \\
eazel & $07 / 29$ & 2,444 & 2,397 & 2,397 & 2.1 & 2,397 & 0 & 0 & 0 & 0 & $0.0 \%$ \\
fileforum & $08 / 31$ & 6,141 & 1,917 & 1,902 & 13.4 & 1,236 & 525 & 5 & 4 & 132 & $0.0 \%$ \\
filehorse & $08 / 04$ & 435 & 351 & 350 & 15.1 & 315 & 17 & 0 & 11 & 7 & $99.1 \%$ \\
portalprogramas & $10 / 04$ & 9,223 & 7,140 & 7,102 & 187.2 & 3,720 & 2,514 & 221 & 252 & 395 & $99.9 \%$ \\
freewarefiles & $09 / 03$ & 17,083 & 7,162 & 7,108 & 94.2 & 3,824 & 2,858 & 59 & 140 & 227 & $0.0 \%$ \\
tucows & $09 / 03$ & 22,695 & 22,187 & 22,153 & 206.1 & 17,835 & 3,869 & 0 & 92 & 357 & $99.3 \%$ \\
snapfiles & $08 / 29$ & 3,998 & 3,651 & 3,648 & 42.5 & 2,387 & 1,118 & 0 & 110 & 33 & $18.6 \%$ \\
filecluster & $09 / 03$ & 11,782 & 7,421 & 7,300 & 172.4 & 4,894 & 1,923 & 17 & 310 & 156 & $100.0 \%$ \\
descargarmp3 & $09 / 03$ & 3,551 & 3,530 & 3,530 & 3.2 & 3,530 & 0 & 0 & 0 & 0 & $0.0 \%$ \\
fileguru & $08 / 31$ & 5,552 & 1,653 & 1,632 & 13.5 & 532 & 814 & 53 & 18 & 215 & $100.0 \%$ \\
download3000 & $09 / 03$ & 967 & 281 & 275 & 2.1 & 207 & 55 & 0 & 4 & 9 & $0.0 \%$ \\
geardownloads & $09 / 06$ & 11,194 & 7,364 & 7,197 & 58.5 & 4,913 & 1,979 & 37 & 23 & 245 & $0.0 \%$ \\
\hline
\end{tabular}

Overall, we downloaded $191 \mathrm{~K}$ programs from the 20 download portals, corresponding to $157 \mathrm{~K}$ unique files with a cumulative size on disk of $2.5 \mathrm{~TB}$. The downloaded files correspond to $65 \%$ of the $325 \mathrm{~K}$ offered programs. Section 3.3.2 details the reasons why a download can fail. The largest download portals are soft112 and softpedia with $107 \mathrm{~K}$ and $69 \mathrm{~K}$ offered programs, respectively. We downloaded the most from softpedia with $48 \mathrm{~K}$ unique files, followed by soft112 with $43 \mathrm{~K}$. The smallest download portals were download3000 and filehorse with less than a thousand programs offered each, and 275-350 unique files downloaded. Note that the download portals are sorted by Alexa ranking (same order as Table 3.2), showing that popularity does not have a direct correspondence with size. For example, uptodown, cnet, softonic, and filehippo all have higher Alexa ranking than the two largest download portals.

File types. Most downloaded files are executables (48\%) and archives (46\%). More specifically, of the unique files downloaded $48 \%$ are EXEs, $40 \%$ ZIP archives, 3\% MSI installers, 2.7\% RAR archives, 1.6\% JAR archives, another 2\% other types of archives (.gzip, .bz2, .7z, .cab) and the remaining are comprised of a long tail of over 70 filetypes including JPEG, text files, ISO images, Office files, PDFs, and source files (e.g., PHP, Python, C). We automatically decompress archives, finding an additional 10M files (170K executables) inside.

Signed executables. Of the 75,615 downloaded executables, 39\% $(29,228)$ are signed. Of those signed executables, $76 \%$ validate correctly on Windows, $20 \%$ have expired certificates, $1 \%$ have revoked certificates, and the remaining $3 \%$ generate various validation errors. There are two download portals (descargarmp3 and eazel) that 
sign all their executables, and each of those two download portals uses a single code signing certificate for signing the executables. We perform an in-depth analysis of these two download portals in Section 3.4.5.

Table 3.3: Percentage of undesirable programs in each download portal.

\begin{tabular}{|r|l|rrr|r|}
\cline { 3 - 6 } \multicolumn{2}{l|}{} & \multicolumn{3}{|c|}{ AV>3 } & AV>0 \\
\hline 1 & Portal & All & PUP & Mal. & All \\
2 & descargarmp3 & $100 \%$ & $100.0 \%$ & $0.0 \%$ & $100.0 \%$ \\
3 & geardownloads & $8.5 \%$ & $5.6 \%$ & $2.9 \%$ & $33.4 \%$ \\
4 & uptodown & $8.3 \%$ & $5.0 \%$ & $3.3 \%$ & $32.1 \%$ \\
5 & tucows & $7.0 \%$ & $4.7 \%$ & $2.3 \%$ & $35.4 \%$ \\
6 & download3000 & $5.9 \%$ & $4.4 \%$ & $1.5 \%$ & $30.2 \%$ \\
7 & filehorse & $5.2 \%$ & $4.3 \%$ & $0.9 \%$ & $20.3 \%$ \\
8 & fileforum & $5.1 \%$ & $3.0 \%$ & $2.1 \%$ & $33.4 \%$ \\
9 & softonic & $4.9 \%$ & $1.9 \%$ & $3.0 \%$ & $30.1 \%$ \\
10 & majorgeeks & $4.8 \%$ & $2.6 \%$ & $2.2 \%$ & $28.8 \%$ \\
11 & filehippo & $4.3 \%$ & $3.3 \%$ & $1.0 \%$ & $21.6 \%$ \\
12 & softpedia & $4.1 \%$ & $1.7 \%$ & $2.4 \%$ & $25.1 \%$ \\
13 & cnet & $3.5 \%$ & $1.3 \%$ & $2.2 \%$ & $25.5 \%$ \\
14 & filecluster & $3.3 \%$ & $2.1 \%$ & $1.2 \%$ & $25.0 \%$ \\
15 & freewarefiles & $2.8 \%$ & $1.4 \%$ & $1.4 \%$ & $25.5 \%$ \\
16 & snapfiles & $2.8 \%$ & $1.8 \%$ & $1.0 \%$ & $26.3 \%$ \\
17 & soft112 & $2.3 \%$ & $1.1 \%$ & $1.2 \%$ & $13.9 \%$ \\
18 & soft32 & $1.6 \%$ & $0.4 \%$ & $1.2 \%$ & $16.8 \%$ \\
19 & fileguru & $1.4 \%$ & $0.5 \%$ & $0.9 \%$ & $15.6 \%$ \\
20 & portalprogramas & $0.2 \%$ & $0.1 \%$ & $0.1 \%$ & $16.5 \%$ \\
\hline
\end{tabular}

\subsubsection{Undesirable Programs in Download Portals}

In this section we examine the prevalence of undesirable programs in download portals. As explained in Section 3.3 we submit to VT all downloaded files larger than $30 \mathrm{MB}$ ( $89 \%$ of all downloaded files). According to the lax policy (i.e., a file is undesirable if at least one AV engine flags it), 41,664 of the files are undesirable. According to the conservative policy (i.e., undesirable if flagged by more than $3 \mathrm{AV}$ engines), 12,340 files are undesirable. Thus, the overall ratio of undesirable programs across all download portals ranges between $8 \%$ (conservative) and 26\% (lax).

We apply AVClass on the 12,340 files flagged as undesirable by the conservative policy in order to classify them as PUP/malware and to label them with a family. Of those, 9,376 (76\%) are PUP and 2,955 (24\%) are malware. These numbers show that PUP is more than three times more common than malware in download portals.

Table 3.3 ranks the download portals by percentage of undesirable programs. For each download portal, it first shows the ratio for all undesirable programs and its split 
Table 3.4: Top 10 PUP and malware families.

\begin{tabular}{|r|l|r|c|r|l|r|}
\hline \multicolumn{3}{|c|}{ PUP } & \multicolumn{3}{c|}{ Malware } \\
\hline Rank & Family & Files & Type & Rank & Family & Files \\
\hline 1 & installcore & 6,033 & PPI & 15 & delf & 50 \\
2 & opencandy & 757 & PPI & 17 & autoit & 38 \\
3 & securityxploded & 202 & DP & 18 & zbot & 32 \\
4 & pswtool & 148 & Generic & 23 & joke & 30 \\
5 & spigot & 100 & Aff. & 29 & scar & 23 \\
6 & prefchanger & 95 & Generic & 32 & bumble & 19 \\
7 & relevantknowledge & 89 & Marketing & 34 & crawler & 19 \\
8 & installmonetizer & 87 & PPI & 36 & rbot & 17 \\
9 & installmonster & 77 & PPI & 37 & atraps & 16 \\
10 & outbrowse & 72 & PPI & 38 & ircbot & 16 \\
\hline
\end{tabular}

into PUP and malware using the conservative policy. Then, it shows the overall ratio using the lax policy. Two download portals (eazel and descargarmp3) have a ratio of $100 \%$ undesirable programs. We examine these two portals in more detail in Section 3.4.5. The other 18 portals have at most $8.5 \%$ undesirable programs.

Table 3.4 shows the top 10 PUP and malware families output by AVClass. For each family it shows its rank, family name, and the number of files in the family. For PUP families we also provide a type. Of the top 10 PUP families, 5 are PPI downloaders, two are generic PUP classes such as password tools (pswtool) and software that changes browsing preferences (prefchanger), one is a label for software downloaded from a specialized download portal (securityxploded), another is a PUP running an affiliate program (spigot), and the last is a marketing tool used to monitor users' Internet habits (relevantknowledge). The lower ranks of malware families, as well as the presence of multiple not-so-popular malware families, also point to PUP abusing download portals for distribution much more often than malware.

Overall, our results identify two portals that are clearly malicious with all programs being considered PUP, and that the amount of undesirable programs in the rest is moderate ranging between $8.5 \%$ and as low as $0.2 \%$. Among the undesirable programs PUP $(76 \%)$ dominates malware $(24 \%)$. These prevalence numbers are in contrast with prior reports that measure much higher rates of undesirable programs among the top downloads and promoted programs $[1,23,25,48]$. We believe that our analysis, which takes into account the complete list of programs in a download portal, rather than only the top downloads, provides a more accurate estimate of the prevalence of undesirable programs in download portals.

\subsubsection{Abusive Behaviors}

In this section we describe several abusive behaviors that we have observed in our analysis. 
Same file as different programs. One behavior that we observe is the same file (i.e., same hash) appearing as different programs in different download portals, and even within the same download portal. In some cases, these are benign programs such as the same Realtek audio driver being offered for different HP notebook models. But, oftentimes these are undesirable programs that fraudulently advertise themselves as different tools. For example, in soft 112 one file is offered as 47 different programs including "start up business advisor", "consumer credit authorisation", "debt collection service london", "outsourcing service wimbledon", and "fsa compliance". Similarly, in uptodown there is a file offered under 6 different author and program pairs such as "bittorrent sync by Bittorrent Inc", "mobaxterm by Mobatek", and "photofilm by KC Software". We argue that these cases where the same file is registered as different programs within the same download portal are easy to identify by the download portal and such registrations should be blocked as they are misleading to users.

The case where the same file is advertised as different programs in different download portals is harder to protect against unless download portals share program information or monitor each other. An example of this case is a file advertised as 10 different programs in 5 download portals including "scftp" in softpedia, "ftpright" in freewarefiles, "esftp" in geardownload, "robust ftp \& download manager" in fileforum, and "free ftp and download manager" in download3000.

External program hosting. A surprising observation is that half of the download portals host less than half of the programs they index, and 35\% of download portals do not host any files. This is shown in the rightmost column in Table 3.2, which captures for each download portal, the percentage of onsite hosted programs. Of the 20 download portals, 10 host nearly all programs (over 98\%) onsite, 7 host no programs onsite, and the other 3 host $14 \%-40 \%$ programs onsite. External hosting of programs allows a malicious publisher to abuse time-of-check to time-of-use (TOCTOU) conditions by submitting a program URL that initially points to a benign file, but later points to an undesirable file. Overall, of the 12,340 undesirable files, $33 \%$ are hosted onsite, and $67 \%$ offsite. However, if we exclude the two download portals that exclusively serve PUP from an external site, the remaining undesirable files are hosted $63 \%$ onsite and $37 \%$ offsite. Thus, while we do observe instances of this behavior, it does not seem that attackers currently are widely abusing such TOCTOU conditions. Still, we advise download portals to periodically scan the externally hosted files.

Impersonating benign authors. The program author advertised in a download portal may not necessarily be the true author since it is hard for download portals to verify authorship in the general case. Thus, some authors may be tempted to impersonate popular benign authors to make their programs more attractive, including malicious authors that want to make their undesirable programs look innocuous. On the other hand, impersonating an author with low reputation does not provide a benefit to the real author. 
Table 3.5: Top 10 undesirable authors with more than 50 files.

\begin{tabular}{|r|l|r|r|r|r|r|r|r|}
\hline Rank & Name & DP & Files & Signed & Pub. & All & PUP & Mal. \\
\hline 1 & zebnet & 74 & 74 & 2 & $68 \%$ & $15 \%$ & $53 \%$ \\
2 & myplaycity & 7 & 100 & 67 & 1 & $49 \%$ & $49 \%$ & $0 \%$ \\
3 & securityxploded & 11 & 397 & 1 & 1 & $48 \%$ & $39 \%$ & $9 \%$ \\
4 & freeridegames & 1 & 73 & 73 & 1 & $48 \%$ & $47 \%$ & $0 \%$ \\
5 & siteken & 1 & 314 & 0 & 0 & $41 \%$ & $31 \%$ & $10 \%$ \\
6 & xilisoft & 10 & 142 & 56 & 2 & $31 \%$ & $31 \%$ & $0 \%$ \\
7 & adobe & 10 & 127 & 48 & 3 & $17 \%$ & $17 \%$ & $0 \%$ \\
8 & nirsoft & 16 & 438 & 33 & 2 & $16 \%$ & $13 \%$ & $3 \%$ \\
9 & mediafreeware & 4 & 85 & 9 & 1 & $15 \%$ & $12 \%$ & $4 \%$ \\
10 & microsoft & 17 & 1930 & 1156 & 21 & $4 \%$ & $4 \%$ & $<1 \%$ \\
\hline
\end{tabular}

Table 3.5 shows the top 10 undesirable authors with more than 50 files across the 20 download portals. For each author, it shows the number of download portals where it appears, the number of files and signed executables, the number of publishers signing the executables, the total percentage of undesirable downloaded files and classified as PUP or malware.

The table includes two benign software publishers: Microsoft and Adobe. A closer look shows that a significant fraction of the files that claim authorship from Adobe and Microsoft are not signed, which is rare for those publishers. This likely indicates other authors trying to impersonate Microsoft and Adobe. Of the programs that claim the author is Adobe or Microsoft $17 \%$ and $4 \%$ are undesirable, respectively. The majority of those are signed by Delivery Agile, a PUP company analyzed in Section 3.4.5.

Repetitive abusers. Another observation from Table 3.5 is that there exist authors that repeatedly abuse download portals (as mentioned earlier, it does not seem likely that other authors are impersonating authors with low reputation). It should be easy for download portals to identify such repetitive abusers, e.g., through the use of blacklists. We observe that the failure to identify them is widespread, rather than being specific to a few careless download portals. The data shows that the majority of those authors have programs in multiple download portals, and only freeridegames and siteken abuse a single download portal (namely tucows).

\subsubsection{Program Execution}

We configured Cuckoo to run programs for 180 seconds. Determining if an installation has successfully completed is challenging as we do not even know how many programs (or which programs) will be installed. We consider an installation successful if the original executable run by Cuckoo (i.e., the installer) writes to disk at least another executable with a different hash, i.e., installs at least one program. Overall, $68 \%$ of the executions install at least one program. 
We observe a significant number of modifications to installed browsers. Our VM has both Internet Explorer (IE) and Firefox installed with IE set as the default browser. We observe 1,399 installers that modify the start page of IE. Of those, $77 \%$ set the homepage to http: / / www. ihot see. com/ and $4 \%$ to http: // search. conduit. com/. We also observe 9 installers that change the default browser, 8 of them to the Avant Browser [112] and one to the now defunct Sundial browser.

We also observe 551 installers that install additional browser functionality. More specifically, 445 installers add an IE toolbar, 20 add a Firefox extension, 5 add an IE bar, 178 add a default URLSearchHook for IE (which hooks any URL typed by the user without a protocol, e.g., to redirect to a hijacker's webpage), and 21 install an IE Browser Emulation. The large difference between Internet Explorer and Firefox modifications is likely due to IE being the default browser.

\subsubsection{Case Study: Vittalia Download Portals}

The download portals eazel and descargarmp3 have noticeable similarities among themselves that distinguish them from the other download portals. Specifically, both download portals offer only executables (no archives or other filetypes), each downloaded file is unique (no file is downloaded twice in those portals), each file downloaded from the same download portal has the same size (the size differs among the two download portals), all executables are signed, and the same code signing certificate is used to sign all executables from each download portal.

The fact that each downloaded file is unique and has the same size points to the all downloads being polymorphic variants of the same program. We confirm this by running the executables in our sandbox. For both download portals, all files show the typical behavior of a PPI downloader [21]. First, it asks the user if he wants to download the original program that the user thought it was downloading from the download portal. If the user declines to install at this point, nothing is installed. Second, it offers to install third party programs such as McAfee WebAdvisor, Avast antivirus, and Pro PC cleaner. For each offer, the user has the option to accept or decline the installation. If the user accepts the offer, another offer is displayed, until no more offers are left. If the user declines one offer, no more offers are shown. Third, all accepted offers are downloaded showing a progress bar. When the download finishes, there is an additional offer. Fourth, the user is asked if he wants to perform the installation of all the accepted offers or do it later. If the user postpones installation, the PPI downloader is still installed and will periodically remind the user to install the programs.

After the process above, the user ends up with the following programs installed: the original program it wanted to download, any offers that it accepted to install, and the PPI downloader. Note that the PPI downloader was never offered to the user, so at a minimum the user always gets an additional program that it did not desire or accepted to install.

All executables downloaded from these two download portals were unknown to 
VT when first downloaded, which can be expected since they seem to be generated on the fly as the user (or our crawler) requests them. However, once submitted to VT all of them were flagged as undesirable by more than $3 \mathrm{AV}$ engines. Furthermore, if we run the executables from these download portals on a Cuckoo sandbox with no anti-anti-evasion techniques, they exhibit a different behavior. In this situation, executables downloaded from eazel install a program called Remebeher and those from descargarmp3 a program called Liret. No third-party offers are shown to the user. This change of behavior indicates anti-sandboxing checks.

Both download portals state in their terms and conditions that they belong to Vittalia Internet, a known PUP publisher that used to run a PPI program called OneInstaller [5], which seems defunct. We query the IP addresses of those 2 portals using VirusTotal and discover another 12 Vittalia download portals hosted on those IP addresses such as solodrivers.com, filewon.com, fileprogram.net, and downloadsoft.nl. The PPI downloader offered by the Vittalia download portals is not the one from the OneInstaller PPI that Vittalia used to run. Instead, the AV engines identify them as the PPI downloader for InstallCore, an Israeli PPI program [5]. In addition, executables downloaded from eazel are signed by "FunnelOpti (Alpha Criteria Ltd.)" and those downloaded from descargarmp3 are signed by "Delivery Agile (New Media Holdings Ltd.)". Both New Media Holdings Ltd. and Alpha Criteria Ltd. are companies part of the IronSource group, who owns the InstallCore PPI program.

Finally, we observe that all files downloaded from eazel are hosted offsite at www. sendchucklebulk.com and those downloaded from descargarmp3 come from 3 domains: www.sendcapitalapplication.com, www. quickbundlesnew.com, and www.guardmegahost.com. Those four domains all resolve to the same set of 6 IP addresses and are registered by the same privacy protection service in Israel. We believe these domains belong to InstallCore. When a user requests to download a file, the download portals request InstallCore's API to generate on the fly a user-customized PPI downloader.

To summarize, our investigation shows that Vittalia has moved away from its own PPI service and instead has signed up as a publisher to the more popular InstallCore PPI service. When a user tries to download any program from one of Vittalia's download portals, they are instead provided an InstallCore PPI downloader generated on the fly for the user. The user may decide to install some offers from third-party advertisers who pay InstallCore for distribution, and Vittalia gets a payment for each installation it enables. The user ends up installing not only the original program that it wanted, but also the PPI downloader, and any offers it accepts. This case study illuminates how some download portals are exclusively used to assist in the distribution of PPI downloaders and PUP products. 


\section{PUP Detection through Installation and Uninstallation Behaviors}

\subsection{Introduction}

Potentially Unwanted Programs (PUP) are a category of undesirable software, that while not outright malicious, contain behaviors considered harmful by many users. PUP includes adware that agressively pushes advertisements and rogueware that scares users into buying a software license, despite its limited functionality. Recent works have established the wide prevalence of PUP measuring that ad-injectors affect $5 \%$ of unique daily IP addresses accessing Google [4], that 54\% of hosts running a popular AV have had some PUP installed [5], and that SafeBrowsing flags over 60 million PUP downloads every week, nearly three times that of malware [21].

While many malware detection systems have been proposed (e.g., [58, 61, 62, 113, 114]), currently, no PUP detection system has been proposed to automatically determine if an input executable is PUP. This is likely due to the different behaviors associated with PUP. Some companies such as Google [11], Microsoft [10], and MalwareBytes [12] have policies for defining what behaviors make a program PUP. A large part of those policies focuses on behaviors related to how a program is installed and uninstalled. For example, Google's policy classifies PUP behaviors into five classes: transparent installation and upfront disclosure, simple removal, keeping good company, clear behavior, and snooping. Of those, the first three are related to program installation and uninstallation.

Windows programs are often distributed as a stand-alone installer executable (e.g., setup.exe), an auxiliary program responsible for performing all installation steps for the target program. There exist custom installers developed by the authors of the target program, as well as installer builder software (or builders), i.e., third-party software that facilitates the creation of installers [115-119]. In addition to the target program, the installer may also install an uninstaller, a stand-alone file (e.g., uninstall.exe) that performs the program's uninstallation. Identifying PUP behaviors during installation and uninstallation requires analyzing the program's installer and uninstaller. 
Installers create problems to static malware detectors. The target programs to be installed may be downloaded from the Internet (thus are unavailable during static analysis) or, when bundled with the installer, may be compressed and even encrypted (thus hidden to the static analysis). In fact, popular ransomware families such as Cerber, Locky, Teerac, Crysis, CryptoWall, and CTB-Locker have started being distributed as installers with an encrypted payload to avoid detection and analysis [120].

Existing malware detection systems based on dynamic analysis can potentially analyze any executable including installers and uninstallers. However, they have two main limitations that prevent them from detecting some of the aforementioned PUP behaviors. First, they analyze a single execution. However, PUP behaviors associated with uninstallation, e.g., whether the uninstallation is complete, require joint analysis of an installation execution and at least one uninstallation execution, e.g., for correlating the installation and uninstallation system modifications.

Second, they work at the granularity of a whole execution or a single executable/process. Operating at whole-execution granularity is problematic because prior work has shown that pay-per-install (PPI) schemes, where a software publisher pays a third party (e.g., another software publisher or a PPI service) to distribute its program, are popular in the distribution of both malware [55] and PUP [5,21]. The widespread use of PPI means that when running a malware or PUP executable, multiple other malware or PUP programs may be downloaded, installed, and executed. Thus, behaviors from multiple processes, possibly from unrelated programs, are combined into a single behavioral report. Operating at executable/process granularity is similarly problematic. For example, installers built using popular builders like NSIS and InnoSetup use multiple processes to install a program. In another example, an installer may install multiple programs, each with its own system modifications and its own uninstaller. A better granularity for detecting PUP is analyzing the installaed programs. However, identifying the list of installed programs, is challenging because Windows does not centrally manage installed programs, e.g., it is optional to let Windows know about a program by adding it to the "Add/Remove Programs List". so that they can later be individually executed to analyze their behaviors.

In this work we present our platform, the first dynamic behavioral detection system that focuses on automatically detecting PUP. Our platform focuses on analyzing the PUP installation and uninstallation behaviors of an input executable. Two salient features of our platform are that it correlates the installation and uninstallation executions to determine if uninstallation is complete and that it operates at the program-level, i.e., it identifes the list of programs installed Our platform runs the input executable, identifies the list of programs installed and whether each program has an uninstaller. For those programs that have an uninstaller, it executes the uninstallation command and correlates the installation and uninstallation system modifications to determine if the uninstallation was complete. 


\subsection{Overview}

Most programs need to be installed before they can be executed. The installation process can be complex. It may need to check if system requirements and dependencies are met, identify and uninstall older program versions, write program files into a specific folder structure, install device drivers, generate configuration files, configure persistent services that run automatically (e.g., by adding Windows registry keys or a cron job), download resources from the Internet, make the program easy to launch (e.g., by creating links or shortcuts), activate the software, check for available newer versions, and so on. Similarly, the uninstallation process can also be complex, as it needs to revert the installation steps.

To ease the installation process for the user, programs are often distributed as a stand-alone installer file, i.e., an auxiliary program responsible for performing all installation steps for the target program. This is particularly common for Windows programs, where the installer is typically a stand-alone executable (e.g., setup.exe), or a MSI installer package [121]. The installer may contain all resources needed to install the target program (e.g., executable, libraries, configuration files, images) or may download some of those resources from the Internet. The embedded resources may be compressed, or even encrypted/packed, which make it difficult to identify them statically. In addition to the target program, the installer may also install an uninstaller, a stand-alone file (e.g., uninstall.exe) that performs the program's uninstallation. It is possible for the same file to be both the program's installer and uninstaller, e.g., by using different command line options.

An important property of an installation is whether it is attended, i.e., requires human interaction, or unattended, i.e., does not require human interaction. Unattended installations typically apply the default installation options selected by the program developer and are useful for system administrators that want to automate the installation of a program on multiple hosts. A special case of unattended installations are silent installations, where the user is not notified that the installation is taking place, i.e., there are no windows or messages presented to the user. Silent installations can be abused by undesirable programs to hide the installation of third party software from the owner of the host.

\subsubsection{Installer Builder Software}

For a developer, building a custom installer and uninstaller for his program can be a complicated process. Thus, commercial and open source installer builder programs have been developed to ease the process. Installer builders enable creating highly customizable installation and uninstallation processes. This is often achieved by defining a scripting language that allows the developer to specify installation and uninstallation steps. Furthermore, installer builders often support plugins [122] so that common steps can be developed once and be easily reused by other developers.

Table 4.1 summarizes the installer builders for Windows programs that our plat- 
Table 4.1: Installer builders detected by AVCLASS.

\begin{tabular}{|l|l|c|c|c|}
\cline { 4 - 5 } \multicolumn{2}{c|}{} & \multicolumn{2}{c|}{ Output } \\
\hline Builder & Publisher & OSS & EXE & MSI \\
\hline InnoSetup [115] & Caphyon Ltd & - & $\checkmark$ & $\checkmark$ \\
InstallMate [124] & Jordan Russell & $\checkmark$ & $\checkmark$ & - \\
InstallShield [118] & Tarma Software & - & $\checkmark$ & $\checkmark$ \\
NSIS [116] & Flexera Software & - & $\checkmark$ & $\checkmark$ \\
Setup Factory [119] & Nullsoft & $\checkmark$ & $\checkmark$ & - \\
Wise & Indigo Rose Sof. & - & $\checkmark$ & - \\
WiX [125] & Wise Solutions & - & $\checkmark$ & $\checkmark$ \\
\hline
\end{tabular}

form detects. For each builder, it presents the publisher, whether the builder is open source, and if it can build EXE and MSI installers. NSIS and InnoSetup are the most popular open source builders. WiX is also open source although less popular. The rest are commercial solutions that require a paid license ${ }^{1}$. All installer builders support the creation of EXE installers, while MSI is only supported by commercial builders and WiX.

Another way to distribute programs are self-extracting (executable) archives produced by popular compression tools such as WinZip or 7-zip. Self-extracting archives do not perform any installation process beyond decompressing (and possibly decrypting) the archive contents into the hard disk and optionally running an installer inside the archive after decompression.

\subsubsection{Installation Monetization}

A popular monetization mechanism for software developers is, during the installation process of their program, to offer to the end-user to install third-party programs. If the end-user accepts to install a third-party program, the publisher of that program will pay the developer some commission for the installation.

There exist several models for such pay-per-install distribution agreements. One model is a direct agreement between two software publishers such as Adobe Systems agreeing to distribute the Google Toolbar with various Adobe products [126]. Another model are pay-per-install (PPI) services [5,21], where the PPI service acts as an intermediary between advertisers that want to distribute their programs and affiliates that own programs that users want to install. The affiliate provides its program to the PPI service and obtains in return an installer that while installing the affiliate's program will offer some advertised programs to the end-user. The affiliate distributes the installer to end-users interested in its software. If the end-user installs an advertised program, the advertiser pays a commission to the PPI service, who in turn pays a frac-

\footnotetext{
${ }^{1}$ Wise Solutions was acquired by Altiris, Inc. in 2003, then acquired by Symantec in 2007 . The Wise installer is now discontinued.
} 
tion of that comission to the affiliate. Yet, another model are affiliate programs where a software publisher looks directly for affiliates that will help distribute its software, without the involvement of a PPI service [5]. Similar to the PPI model, affiliates obtain a commission for each installation of the advertised program.

Regardless of the distribution model, the advertised programs can be embedded in the affiliate's installer or downloaded from the Internet. In the PPI model, it is common for the installer to be produced by the PPI service. During installation, the PPI installer connects to the PPI service to download the current list of advertised programs, taking into account factors like the geographical location of the end-user [21].

\subsubsection{Enabling User Uninstallation}

An important property of an installed program is that the user should be able to easily and completely uninstall it. In fact, if a program cannot be uninstalled, is difficult to uninstall, or does not completely uninstall itself, then it would be considered PUP according to popular PUP guidelines [10-12].

There are three main requirements that need to be satified to enable a user to uninstall a program: the user should know that the program is installed, the program should provide an uninstallation command, and the user should know how to invoke the uninstallation command.

The recommended approach to satisfy those three requirements is to register the installed program so that it appears in the Add/Remove Programs list under the Control Panel. Registering a program requires that during installation the program creates some registry keys under HKEY_LOCAL_MACHINE \SOFTWARE $\backslash$ Microsoft \Windows \CurrentVersion\Uninstall\PROGRAM where PROGRAM is a unique identifier for the program, e.g., a program name or a GUID. The two main keys that need to be created under that registry path are DisplayName whose value is the program name shown in the list of Add/Remove programs and Uninstallstring whose value is the uninstallation command for the program. Many other registry keys can be created under that path to provide additional program information such as version, publisher, language, install date, install path, information URL, and contact information [127]. However, only those two keys are needed to satisfy the above requirements. Popular installer builder software in Table 4.1, as well as the Windows Installer service if an MSI package is used [128], register by default the installed program by creating those registry keys. However, it is possible for the developer to configure the installer to avoid registering an installed program.

An alternative way to satisfy the above three requirements, without registering the program under Add/Remove programs, is for the program to add links to itself in a visible location (e.g., the Start Menu or the Desktop), to provide an uninstaller, and to add a link with the command to pass to the uninstaller in the same visible location. Any other uninstallation procedures likely violate the above three requirements and make it difficult for the user to perform an uninstallation. 


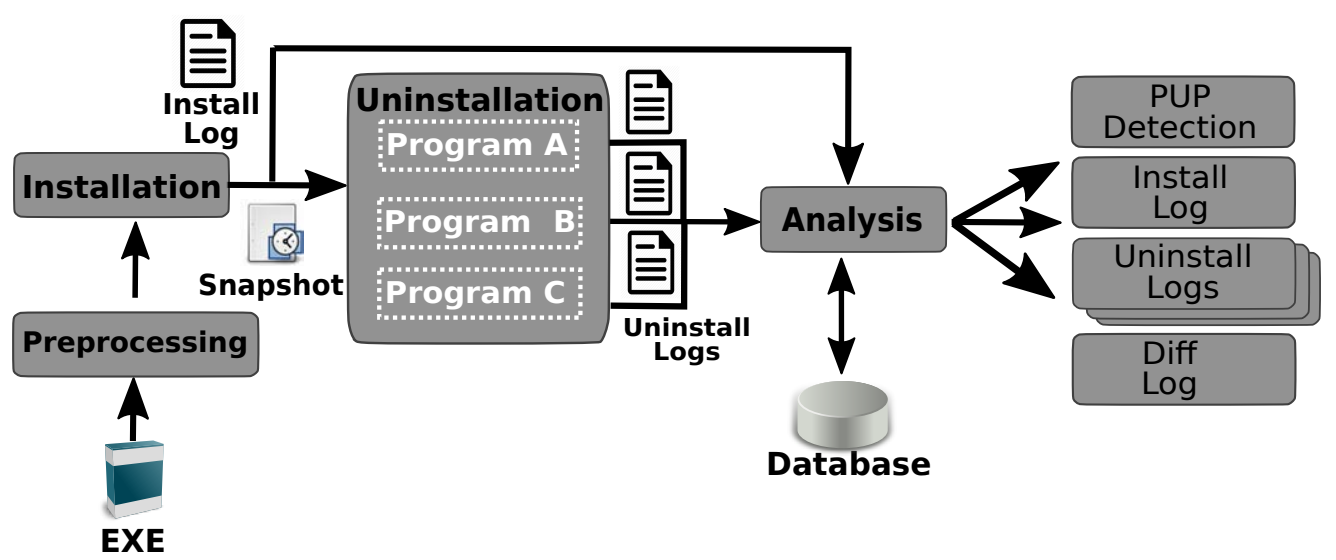

Figure 4.1: High level architecture.

\subsubsection{PUP detection}

The goal of our platform is to detect PUP installation and uninstallation behaviors. In particular, these are the PUP behaviors our platform detects:

- Install: Program(s) installed but no window open

- Install: Installation impacts unrelated content (e.g., system content)

- Install: Bundling with detected program

- Install: Dangerous changes without notification

- Uninstall: No uninstaller

- Uninstall: Difficult to find uninstaller

- Uninstall: Incomplete uninstallation

- Uninstall: Uninstallation impacts unrelated content (e.g., system content)

- Uninstall: Uninstallation adds new content

In addition to the above behaviors, our platform can also detect other PUP behaviors that are not specific to our platform. For example, our platform includes in the installation log whether the installer is (properly) signed as the Google guidelines require, but other works have addressed PUP and malware code signing [2]. Our platform can also be used to analyze behaviors during program execution (as opposed to during installation and uninstallation), but many systems already exist for that purpose, e.g., $[58,60-62]$. 


\subsubsection{Approach Overview}

Figure 4.1 shows a high-level overview of our approach. It takes as input a Windows executable and comprises four modules: pre-processing, installation, uninstallation, and analysis. The pre-preprocessing module first collects static information from an input executable including file hashes, file size, filetype, code signing information, and VirusTotal (VT) report [33] with AV detections.

After pre-processing, the installation module runs the executable in a virtual machine (VM) trying to complete a successful installation, e.g., by automatically interacting with the windows presented. During the installation, detailed information about the installation behaviors are collected including all processes created; all windows and window components shown to the user; files created, removed, modified; registry keys created, removed, modified; and so on. At the end of the installation, the $\mathrm{VM}$ is rebooted to allow the installation to complete any remaining steps. After rebooting, a snapshot of the VM with the installed program is taken. The installation outputs the snapshot and an installation log that identifies the programs installed. For each installed program it determines whether an uninstaller exists and its uninstallation command.

If the executable did not install any program (i.e.,not an installer or installation failed), or if none of the installed programs have an uninstaller, the process completes and outputs the installation log.

For each installed program with an uninstallation command, the uninstallation module tries to uninstall it, producing an uninstall log with the same behavioral information on processes, files, and registry keys collected during the installation. The uninstallation takes as input the VM snapshot with the installed program, as well as the uninstallation commands.

The installation log and all uninstallation logs are input to the analysis module, whose goal is to identify PUP behaviors. First, it examines for PUP behaviors in the installation such as installations with no GUI and unexpected system modifications. Then, it correlates the installation log and the uninstallation logs to determine which installed programs were not uninstalled at all, and for those that were uninstalled, how complete the uninstallation was. This information is output in a diff log.

The output of our platform is an installation log, possibly a set of uninstallation $\log$, a diffing $\log$, and a determination of whether the input executable is PUP.

\subsection{Program Execution}

Program execution is common to the installation and uninstallation modules. It runs an input executable (i.e., an installer or an uninstaller) and tries to complete the installation or uninstallation by interacting with the graphical user interface (GUI) windows (Section 4.3.1) while collecting behavioral information (Section 4.3.2). During installations, it also determines the builder of identified installers (Section 4.3.3). It outputs an installation or uninstallation log with the behavioral information on the execution. 


\subsubsection{Automated User Interface Interaction}

Most installers (and uninstallers) require GUI interaction to prevent the installation (or uninstallation) process to stall. Automating the interaction to allow the installation to advance is an important feature for analyzing installers.

Our policy for GUI interaction is to simulate a user that clicks yes/next on any window presented, so that the installation proceeds quickly. By introducing clicks on the window elements (e.g., buttons) that advance the installation, the approach works with different GUI implementations (e.g., Windows native and COM APIs, .NET libraries, XAML, Qt, Sciter). Our GUI interaction module builds on top of the one used by Cuckoo [60]. Cuckoo's module iterates on all windows looking for button elements. For each button found, it extracts its label and compares it with a whitelist of button labels it should click and a blacklist of button labels to avoid clicking. However, Cuckoo's implementation has a low $40 \%$ success ratio in our evaluation. We have extended its functionality with the following changes that increase the success ratio to $86 \%$.

- We have extended Cuckoo's button whitelist and blacklist with new button labels. Furthermore, Cuckoo includes only English labels, so we have translated all labels to other languages, e.g., French, German, and Spanish.

- Alert boxes can be displayed by the program using a new window or a dialog box. Cuckoo only supports the new window implementation and the exploration stalls when a dialog box needs to be clicked. Thus, we have added support to identify and click on dialog boxes.

- Cuckoo iterates on all windows including system windows such as the Start menu and the notification bar. It often happens that Cuckoo's interactions get stuck on system windows that do not advance the installation. Thus, we have modified it to focus only on active (i.e., installation) windows, ignoring system windows.

Despite these improvements, $14 \%$ of installations still do not successfully complete. We plan to continue improving the GUI interaction module and to eventually contribute our improvements to the Cuckoo project.

\subsubsection{Behavioral Information}

Table 4.2 summarizes the behaviors monitored during program execution. For each window presented to the user, our platform collects a screenshot and identifies their textual elements: title, button labels, and text elements. For directories it collects their path, and for files and registry keys their path, name, and value. It also tracks file links created in the Desktop, Start Menu, and Quick Launch bar, as well as Windows tasks (similar to Linux cron jobs) that are defined as binary job files placed in a OS directory. Finally, it collects a network trace of the communication. 
Table 4.2: Behaviors monitored during execution.

\begin{tabular}{|c|c|c|c|c|}
\hline \multicolumn{2}{|c|}{ Behavior } & Create & Modify & Remove \\
\hline \multicolumn{2}{|c|}{ Process } & $\checkmark$ & $\checkmark$ & $\checkmark$ \\
\hline \multicolumn{2}{|l|}{ File } & $\checkmark$ & $\checkmark$ & $\checkmark$ \\
\hline \multicolumn{2}{|c|}{ Directory } & $\checkmark$ & & $\checkmark$ \\
\hline \multicolumn{2}{|c|}{ Registry Key } & $\checkmark$ & $\checkmark$ & $\checkmark$ \\
\hline \multicolumn{2}{|c|}{ Network } & $\checkmark$ & & \\
\hline Window & $\begin{array}{l}\text { Title } \\
\text { Button labels } \\
\text { Text elements } \\
\text { Screenshot }\end{array}$ & $\begin{array}{l}\checkmark \\
\checkmark \\
\checkmark \\
\checkmark\end{array}$ & & \\
\hline Link & $\begin{array}{l}\text { Start Menu Link } \\
\text { Desktop Link } \\
\text { Quick Launch Link }\end{array}$ & $\begin{array}{l}\checkmark \\
\checkmark \\
\checkmark\end{array}$ & & $\begin{array}{l}\checkmark \\
\checkmark \\
\checkmark\end{array}$ \\
\hline Task & & $\checkmark$ & & $\checkmark$ \\
\hline
\end{tabular}

The program execution builds a process tree initialized with the input executable and to which each process created by an already tracked process is added. Each behavior in Table 4.2 is annotated with the identifier of the process (i.e., the PID) that produces it. Only behaviors produced by processes in the process tree are considered, i.e., system modifications performed by unrelated OS processes are ignored.

\subsubsection{Installer Classification}

For each installed program, our platform uses behavioral signatures to determine if its installer has been built using one of the builders in Table 4.1. While there exist filetype identification tools that identify installers using static signatures, those tools only detect installers from the most popular builders and cannot identify custom installers. For example, the popular TrID [129] tool only identifies 4 out of 11 builders in Table 4.1 and cannot identify custom installers. Static tools also have the problem that a installer from one builder can embed an installer from a different builder. In those cases, the installer file matches signatures from multiple builders.

In our work, an installer is a file that is executed during a run and it creates at least one executable with a hash different than itself. This generic behavioral rule allows to identify any installers in a run including custom installers. To further classify installers by builder, our platform uses $\mathrm{Y}$ behavioral signatures. Those behaviorals signatures capture that installers create specific directories, installation log files, and registry keys. and that they may publicize the builder on the title of GUI windows. For example, InstallMate creates a README.txt file that contains the string "Installmate". Other builders such as NSIS use plugins that are written to a temporary directory with a specific name. For open source builders, we have built a whitelist of plugin hashes obtained by downloading all the builder versions from their repository. 
For each executed file that creates other executables (i.e., for each installer), if its process matches the behavioral rules of a builder, the file is classified as a installer of that builder type. Otherwise, the file is considered a custom installer.

\subsection{Identifying Installed Programs}

After an installation run completes, the installation module identifies the list of installed programs. An installed program comprises the files that belong to the program; the installation paths where those files are located; the links to those files in the Desktop, Start Menu, and Quick Launch bar; the tasks associated to files from the program; the installers that installed the program; the program uninstallation commands (if any); and the possible program names.

Identifying the installed programs is challenging because Windows does not keep track of the installed programs and because installers typically run with administrative privileges and can thus freely modify the system. At a high level our algorithm partitions the list of created and modified files into candidate programs and filters out those candidates that do not correspond to real programs, e.g., auxiliary installer files and resources modified by the installed programs. In detail, our algorithm comprises five steps: pre-filtering, partitioning, identifying uninstallation commands, merging, and post-filtering. We detail these five steps next.

\subsubsection{Pre-Filtering}

As mentioned in Section 4.3.3, the processes in the process tree can be classified into installer processes that create or modify at least one executable, and non-installer processes. Note that here we use the word executable broadly to include any active code such as executables (.exe), dynamic libraries (.dll), screensavers (.scr), Active X Controls (.ocx), Windows installation packages (.msi), and browser extensions (.xul, .crx). Non-installer processes correspond to installed programs that are executed by its installer after their installation completes or programs already installed in the VM such as Internet Explorer, Java, or Windows processes.

Intuitively, non-installer processes cannot install programs since they do not create any executables. Thus, the pre-filtering step removes all files created and modified by non-installer processes. Performing this pre-filtering before the other steps improves the performance of the identification process.

\subsubsection{Partitioning}

The next step is to create a list of candidate installed programs by partitioning the list of created and modified files output by the pre-filtering using the process that created the files and the directory where they are located. The intuition behind the partitioning by installer process is that a program is most often installed by a single installer because 
using multiple installers requires synchronization between them (e.g., on which installation path to use). The intuition behind the partitioning by directory is that the files of a program are most often installed under the same path. Of course, both above rules are not always true. For example, an installer could be split into two executables, one that performs the initial installation and another that finalizes the installation. As another example, program files may be located outside the main installation path such as Desktop links or kernel drivers installed under $C: \backslash W$ indows $\backslash$ system $32 \backslash d r i v e r s \backslash$. Those cases are handled later in the process. At this step, it is fine to over-split a real program into multiple candidates. The merging step identifies candidates that are really part of the same program.

The partitioning by installer process first splits the list of files created or modified such that files created or modified by the same installer process are in the same process group. Next, the partitioning by installation path handles the common case where an installer installs multiple programs by subdividing the process groups into candidate programs containing files created by the process under the same installation path.

The partitioning by installation path differentiates between files created in a directory that existed before the run (e.g., C: \Windows $\backslash$ system $32 \backslash$ ) and files created in new directories. All files in a pre-existing directory are placed in the same candidate program. For new directories, it extracts the top-level directory with files that did not exist before the run. For example, if there are files created by the same process in $C: \backslash$ ProgramFiles $\backslash$ Mozilla $\backslash F i r e f o x \backslash$ and $C$ : $\backslash$ ProgramFiles $\backslash$ Mozilla $\backslash$ Thunderbird $\backslash$ then it creates two groups one for each subdirectory. In another example, if there are files created by the same process in $\mathrm{C}: \backslash$ ProgramFiles $\backslash$ MyProgram $\backslash$ and $\mathrm{C}: \backslash$ ProgramFiles $\backslash$ MyProgram $\backslash$ component $\mathrm{S} \backslash$ then it creates a single group for the MyP rogram folder.

After the partitioning has completed, an initial list of names is obtained for each candidate program using two rules. If the installation path is a new directory then the directory path minus the pre-existing parent folder is a possible name, e.g., Mozilla Firefox or Mozilla \Thunderbird in the example above. If the installation path is a pre-existing directory, then for each non-DLL executable file in the candidate program it adds the filename without extension, e.g., sstunst 3 for $\mathrm{C}: \backslash W$ indows $\backslash$ system32\sstunst 3 . exe.

The candidate programs output by the partitioning have one installer process, one installation path, the list of all files created by that process under that path, and a list of possible program names.

\subsubsection{Identifying Uninstallation Commands}

Windows keeps the list of installed programs it knows about in the registry under HKEY_LOCAL_MACHINE \SOFTWARE \Microsoft \Windows $\backslash$ CurrentVersion \Uninstall\PROGRAM $\backslash$, where PROGRAM is a program name or GUID. Since it is not mandatory to notify Windows of an installed program, these PROGRAM entries may only correspond to a subset of all installed programs. 
Each PROGRAM entry may have multiple keys, but three of them are most important: DisplayName contains the program name shown in the list of Add/Remove programs in the Control Panel, Uninstallstring contains the program's uninstallation command, and InstallLocation (optionally) contains the program installation path.

For each PROGRAM entry, our algorithm creates a candidate program with the uninstallation command in Uninstallstring, the installation path in InstallLocation (if any), the DisplayName value and the PROGRAM string as two possible names, and an empty list of files. The forthcoming merging step identifies the candidate program corresponding to each PROGRAM entries and merges them.

In addition to the PROGRAM entries in the registry, our algorithm also tries to identify uninstaller files in the candidate programs output by the partitioning step. For this, if a candidate program contains an EXE whose filename contains the substring unins, then the file is added to the list of uninstallation commands. This step helps identifying uninstallers that Windows does not know about, but could still be located by a user who examines the installation path contents.

\subsubsection{Merging}

The goal of the merging step is identifying if multiple candidate programs are part of the same installed program. Merging is a recursive process that examines every pair of candidate programs using five merging rules. If at least one rule is satisfied both candidate programs are merged into a new candidate program with their combined information, i.e., with the union of their installation paths, installer processes, names, uninstallation commands, files, and links. The merging recurses until no more candidate programs can be merged. We detail the five merging rules next.

Merge by installation path. This rule identifies programs installed by multiple installers, i.e., by multiple installer processes. It merges two candidate programs if they write files in the same installation path and that installation path is not a pre-existing directory.

Merge by uninstallation command. This rule identifies the candidate program that corresponds to a PROGRAM entry obtained from the registry. This rule only applies when one of the two candidate programs being examined contains an uninstallation command from the registry (i.e., comes from the step in Section 4.4.3). If so, it first parses the uninstallation command into the uninstaller's path, the uninstaller's filename, and the uninstallation parameters. Then, it checks if the the uninstaller's path is among the installation paths of the other candidate program, or if the uninstallation parameters string contains one of the installation paths of the other candidate program. If one of these two checks is satisfied, both candidate programs are merged. The second check is needed for uninstallation commands that 
use Windows services. For example, in the uninstallation command regsvr 32 / u Is "C: \ProgramFiles $\backslash 811$ Toolbar $\backslash$ toolbar.dll", the uninstaller is the Windows service regsvr32, but if the the other candidate program has as installation path $\mathrm{C}: \backslash$ ProgramFiles $\backslash 811 \mathrm{To} 01 \mathrm{bar}$ then both candidate programs are merged because the command is uninstalling a service whose file is located in that installation path.

Merge by link target. This rule identifies links to program files that the installation may have created in the Desktop, Start Menu, or Quick Launch bar. In Windows, those links are binary files placed in specific OS directories, i.e., C: \Programdata $\backslash$

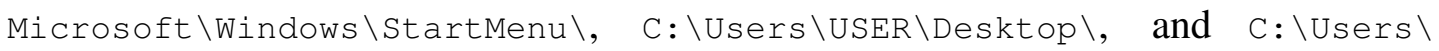
USER $\backslash$ Appdata $\backslash$ Roaming $\backslash$ Microsoft $\backslash$ InternetExplorer $\backslash$ QuickLaunch $\backslash$. Thus, before this step, the links in each of those directories are (incorrectly) considered a candidate program. This rule only applies when one of the two candidate programs has one of those three installation paths. If so, the contents of each link file (e.g., there may be multiple Desktop links) are parsed to extract the target, which is itself parsed into a filepath, a filename, and parameters. If the extracted filepath is among the list of installation paths for the other candidate program then both candidate programs are merged.

Merge by task target. This rule identifies Windows tasks to periodically execute program files. Windows tasks are specified using binary job files (.job) placed in a OS directory: $\mathrm{C}: \backslash W$ indows $\backslash$ tasks $\backslash$. This rule only applies when one of the two candidate programs being examined has that installation path. If so, the contents of all job files are parsed to extract their targets, which are then parsed into a filepath, a filename, and parameters. If the extracted filepath is among the list of installation paths for the other candidate program then both candidate programs are merged.

Merge by name. The final rule identifies candidate programs generated by the same installer under different folders, but that have a common possible name. This rule merges the two candidate programs if their set of installer processes and their set of possible names both have non-empty intersections.

\subsubsection{Post-Filtering}

The post-filtering step removes candidate programs that are not real programs, but rather auxiliary files required by the installer and placed in temporary directories. One naive way to implement the post-filtering would be to remove all candidate programs installed in temporary directories. However, we observe that real programs are sometimes installed in temporary directories. To avoid removing those programs, the postfiltering first applies a generic filtering rule, and then specific rules that capture the default behaviors of popular builders. The generic filtering rule filters out a candidate 
program if it only has one installation path, the installation path is under a temporary directory, and the only executable files that it contains are DLLs or whitelisted EXEs. The reason to ignore DLLs is that popular builder such as NSIS use DLL plugins to implement common installation steps.

The following builder-specific rules apply only if the candidate program contains a single (not-whitelisted) EXE. If any of the rules is matched, the candidate program is removed.

- Installer is NSIS and the EXE's filename is the same as the process installer's filename.

- Installer is InnoSetup and the EXE's filename is the same as the process installer's filename, replacing the ".exe" extension with ".tmp".

- Installer is InstallShield and the EXE's filepath ends with \disk1 \setup. exe.

- Installer is WISE and the EXE's filename matches regular expression gl [0$9 a-f]\{5\} \backslash . t m p$.

- Installer is SetupFactory and EXE's filename is irsetup.exe.

Installation log. The list of installed programs output by the above process is incorporated to the execution log to produce an installation log, which captures all information about the installation and is an input to the analysis module. We say that an installation was successful if it installed at least one program.

\subsection{Evaluation}

This section describes the datasets used in the evaluation of our approach, it also presents the information about our installation and uninstallation executions.

\subsubsection{Datasets}

To evaluate our platform we use two datasets of Windows executables. The first portals dataset comprises 16,547 executables downloaded from 14 download portals, e.g., CNET, Softonic, FileHippo, Tucows, and Softpedia. The dataset also comprises a database with the metadata on the collection including the download date, download URL, and program and author name advertised in the download portal. Three noteworthy properties of this dataset are that it contains a large number of installers, that we know the name and author of the program that is expected to be installed, and that it contains benign, PUP, and malware.

The second benign dataset comprises the installers of 54 benign applications that we have manually selected as baseline. It includes, among others, popular browsers 
Chapter 4. PUP Detection through Installation and Uninstallation Behaviors

Table 4.3: Summary of installation runs

\begin{tabular}{lrr}
\hline Property & \multicolumn{1}{c}{ Portals } & \multicolumn{1}{c}{ Benign } \\
\hline All installation runs & $16,547(100.0 \%)$ & $54(100.0 \%)$ \\
with at least one installed executable & $14,835(89.8 \%)$ & $34(62.9 \%)$ \\
with at least one installed program & $12,797(77.3 \%)$ & $13(24.1 \%)$ \\
\hline All processes & $25,428(100.0 \%)$ & $89(100.0 \%)$ \\
Installer processes & $18,332(34.9 \%)$ & $15(16.8 \%)$ \\
\hline All installed programs & $16,216(100.0 \%)$ & $15(100.0 \%)$ \\
with at least one uninstaller command & $12,091(74.56 \%)$ & $4(26.7 \%)$ \\
with more than one uninstaller & $196(1.2 \%)$ & $0(0.0 \%)$ \\
with at least one link & $10,963(67.0 \%)$ & $3(20 \%)$ \\
with at least one job & $2(<0.1 \%)$ & $0(0.0 \%)$ \\
with more than one installer & $251(1.4 \%)$ & $0(0.0 \%)$ \\
with more than one installation path & $7,183(39.7 \%)$ & $2(13.3 \%)$ \\
\hline All files created & $579,973(100.0 \%)$ & $1,508(100.0 \%)$ \\
Executables & $112,498(19.4 \%)$ & $454(30.1 \%)$ \\
Resources & $467,475(80.6 \%)$ & $1,054(69.9 \%)$ \\
Installed program files & $409,511(77.4 \%)$ & $1,133(75.1 \%)$ \\
\hline All directories created & $121,455(100.0 \%)$ & $341(100.0 \%)$ \\
\hline All registry keys created & $945,461(100.0 \%)$ & $606(100.0 \%)$ \\
\hline All windows created & $54,260(100.0 \%)$ & $88(100.0 \%)$ \\
All window elements created & $1,302,735(100.0 \%)$ & $1,591(100.0 \%)$ \\
\hline
\end{tabular}

(Chrome, Firefox, Opera), email clients (Thunderbird, Opera Mail, MailBird), instant messaging applications (Skype, Slack, ICQ), document readers (Acrobat Reader, Nitro PDF, Foxit Reader), text editors (Notepad++, UltraEdit, Atom), image editors (Gimp, PhotoScape, InkScape), multimedia players (VLC, Adobe Flash, Quicktime), and security tools (Wireshark, Nessus, Microsoft Safety Scanner).

\subsubsection{Installation Runs}

Table 4.3 summarizes the information about our installation runs. We performed 16,547 installation runs, each of them of a distinct EXE. Of those, 14,835 runs installed at least one executable and 12,797 runs installed at least one valid program (output by the process in Section 4.4). The runs that did not install a valid program are due to one of two causes: either the executable run is not an installer, but rather a stand-alone application that does not need to install other executables, or the installation failed, e.g., the GUI exploration was not able to successfully complete the installation.

To understand how much we achieved from our GUI exploration improvements, we performed the following experiment. We randomly selected 100 samples from our dataset and run those 100 samples both with an unmodified installation of Cuckoo 2.04, as well as with our platform. Cuckoo off-the-shelf had a success rate of only $40 \%$. With our GUI improvements the success rate climbed to $86 \%$. 
Table 4.4: Split of programs by installer type

\begin{tabular}{lr}
\hline Installer & Count \\
\hline InnoSetup & $42.9 \%$ \\
Unknown & $35.8 \%$ \\
NSIS & $13.8 \%$ \\
WISE & $4.6 \%$ \\
SetupFactory & $1.8 \%$ \\
InstallMate & $0.3 \%$ \\
InstallShield & $0.2 \%$ \\
WiX & $0.1 \%$ \\
Advanced Installer & $0.1 \%$ \\
Setup2Go & $0.1 \%$ \\
WinRAR-sfx & $<0.1 \%$ \\
Setup Specialist & $<0.1 \%$ \\
\hline
\end{tabular}

Table 4.5: PUP detection on 16,547 installations

\begin{tabular}{|l|r|}
\hline Behavior & Value \\
\hline \multicolumn{2}{|c|}{ Installation } \\
\hline Program(s) installed but no window open & $89(0.5 \%)$ \\
Impacts unrelated content(Files) & $279(1.5 \%)$ \\
Impacts unrelated content(RK) & $1,662(9.9 \%)$ \\
Impacts unrelated content(FIles I RK) & $1,795(10.7 \%)$ \\
\hline \multicolumn{2}{|c|}{ Uninstallation } \\
\hline No uninstaller & $4,327(26 \%)$ \\
Difficult to find uninstaller & $269(1 \%)$ \\
Incomplete uninstallation & $4,224(25 \%)$ \\
\hline
\end{tabular}

Table 4.4 shows for all the installed programs, the builder used to build its installer, or Unknown/Custom if the builder could not be identified and thus the installer is likely custom made.

\subsubsection{Uninstallation Runs}

We performed 12,276 uninstallation runs, for the 12,091 programs with at least one uninstaller command. There are more uninstallation runs due to the programs with more than one uninstaller. We determine that only 6,260 programs were completely removed, 4,224 were partially removed, and 3,498 were not removed. Table 4.5 shows behaviors detected during the installation and uninstallation for 16,547 samples analyzed. 


\subsection{Discussion}

Determining if the user gets the desired program. A challenging question is whether an installer installs the program that the user wanted to install. Answering this question requires knowing what program the user wanted to install. In our work we have addressed this by collecting programs from download portals, which enables us to know exactly what program our crawler was downloading from the metadata in the download portal. In other situations, e.g., when the installer comes from a malware feed, we lack such context and we only have the name of installer, which may not have enough information. This highlights the importance of context in malware collection.

Static analysis. This paper proposes a dynamic approach for PUP detection. Installers create problems to static analysis. First, it is difficult to determine if a given executable is an installer without executing it. Second, the target programs bundled with the installer are often compressed, and even encrypted. Static unpackers to extract the installer's contents are specific to one or a few builders (e.g., [130]) or are no longer updated (e.g., [131,132]). Even if a static unpacker can extract the installer's contents, critical information about the installation process cannot be provided including the windows and messages presented to the user, installation paths, the who-installs-who relationship when multiple target programs are installed, and any content downloaded from the Internet. 


\section{PUP and Malware Labeling}

\subsection{Introduction}

Labeling a malicious executable as a variant of a known family is important for multiple security applications such as identifying new threats (by filtering known ones), selecting disinfection mechanisms, attribution, and malware lineage. Such labeling can be done manually by analysts, or automatically by malware classification approaches using supervised machine learning $[17,18,69]$ (assuming the sample belongs to a family in the training set), and also through malware clustering approaches $[27,67,68,133]$ followed by a manual labeling process to assign a known family name to each cluster.

Labeling executables is also important for building reference datasets that are used by researchers for training those malware classification supervised approaches and for evaluating malware clustering results. This creates a bit of a chicken-andegg problem, which prior work resolves by building reference datasets using AV labels [17,27,67-69]. However, AV labels are well-known to be inconsistent [27-29,71]. In particular, $\mathrm{AV}$ engines often disagree on whether the same sample is malicious or not, and the family name that the label encodes may differ, among others, because of lack of a standard naming convention (conventions such as CARO [30] and CME [31] are not widely used), lack of interest (the main goal of an AV is detection rather than classification $[134,135]$ ), using heuristic or behavioral detections not specific to a family, and vendors assigning different names (i.e., aliases) to the same family.

Still, despite their known inconsistencies, AV labels are arguably the most common source for extracting malware labels. This likely happens because in many occasions no other ground truth is available, and because, despite its noisy nature, AV labels often contain the family name the analyst wants. Thus, extracting as accurate family information as possible from AV labels is an important problem.

Several limitations affect the process in which prior work builds family name ground truth from AV labels. First, some approaches use the full AV labels, which is inaccurate because the family name comprises only a fraction of the full label. For example, an AV engine may use different labels for samples in the same family, but 
still assign the same family name in those labels, e.g., when using two different detection rules for the family. Other works extract the family name in the labels through a manual process that is not detailed, does not handle aliases between family names, and does not scale to hundreds of thousands, or millions, of samples.

Second, it has been shown that no single AV engine detects all samples and that the number of AV engines needed to achieve high correctness in the family name is higher than for detection [29]. To address these issues, it is common to resort to majority voting among a fixed set of selected AV vendors. But, this requires selecting some AV vendors considered better at labeling, when prior work shows that some AV vendors may be good at labeling one family, but poor with others [29]. In addition, a majority cannot be reached in many cases, which means a family name cannot be chosen for those samples and they cannot be added into the evaluation or training data [72]. And, focusing on the samples where the majority agrees may bias results towards the easy cases [82]. Furthermore, prior work assumes the results of this process correspond to the ground truth, without quantitatively evaluating their quality.

In this work, we describe AVCLASS, an automatic labeling tool that given the AV labels for a, potentially massive, number of samples outputs the most likely family names for each sample, ranking each candidate family name by the number of AV engines assigning it to the sample. Selecting the top ranked family corresponds to a plurality vote, i.e., family with most votes wins. AVCLASS implements novel automatic techniques to address 3 main challenges: normalization, removal of generic tokens, and alias detection. Using those techniques AVCLASS automatically extracts as precise family information as possible from the input AV labels.

We envision AVCLASS being used in two main scenarios. In the first scenario, an analyst does not have access to a state-of-the-art malware clustering system (e.g., $[27,67,68,133])$. When faced with labeling a large amount of samples, the analyst uses AVCLASS to efficiently obtain the most likely families for each sample. Here, AVCLASS acts as an efficient replacement for both clustering and labeling the resulting clusters.

In the second scenario, the analyst has access to an state-of-the-art malware clustering system and can use AVCLASS for 3 tasks. First, it can use AVCLASS to automatically label the output clusters with the most likely family name used by AV vendors. Second, AVCLASS's output can be used to implement a feature based on AV labels (e.g., whether two samples have the same family name) that can be added to the existing clustering. Thus, rather than assuming that the AV labels constitute the ground truth, the analysts incorporates the AV labels knowledge into the clustering system. Third, AVCLASS's output can be used to build a reference clustering to evaluate the clustering results. Since AVCLASS tags each candidate family name with a confidence factor based on the number of $\mathrm{AV}$ engines using the name, the analyst can select a threshold on the confidence factor for building the reference dataset, e.g., replacing the default plurality vote with a more conservative (majority or else) vote.

The salient characteristics of AVCLASS are: 
- Automatic. AVCLASS removes manual analysis limitations on the size of the input dataset. We have evaluated AVCLASS on 8.9 M malicious samples, larger than any dataset previously used by malware clustering and classification approaches.

- Vendor-agnostic. Prior work operates on the labels of a fixed subset of 1-48 AV engines. In contrast, AVCLASS operates on the labels of any available set of AV engines, which can vary from sample to sample. All labels are used towards the output family name. AVCLASS has been tested on all 99 AV engines we observe in VirusTotal [33], the largest AV engine set considered so far.

- Plurality vote. AVCLASS performs a plurality vote on the normalized family names used by all input engines. A plurality vote outputs a family name more often than a majority vote, since it is rare for more than half of the AV engines to agree.

- Cross-platform. AVCLASS can cluster samples for any platforms supported by the AV engines. We evaluate AVCLASS on Windows and Android samples.

- Does not require executables. AV labels can be obtained from online services like VirusTotal using a sample's hash, even when the executable is not available.

- Quantified accuracy. The accuracy of AVCLASS has been evaluated on 5 publicly available malware datasets with ground truth, showing that it can achieve an F1 score of up to 93.9 .

- Reproducible. We describe AVCLASS in detail and release its source code. ${ }^{1}$

\subsection{Approach}

Figure 5.1 shows the architecture of AVCLASS. It comprises two phases: preparation and labeling. During the preparation phase, an analyst runs the generic token detection and alias detection modules on the AV labels of a large number of samples to produce lists of generic tokens and aliases, which become inputs to the labeling phase. In particular, the generic token detection takes as input the AV labels of samples for which their family is known (e.g., from publicly available labeled datasets $[69,74,136,137])$ and outputs a list of generic tokens, i.e., tokens that appear in labels of samples from different families. The alias detection module takes as input AV labels of a large number of unlabeled samples and outputs pairs of family names that alias to each other.

The labeling phase is the core of AVCLASS and implements the label normalization process. It takes as input the AV labels of a large number of samples to be labeled, a list of generic tokens, a list of aliases, and optionally a list of AV engines to use. For each sample to be labeled, it outputs a ranking of its most likely family names. The

\footnotetext{
${ }^{1}$ https://github.com/malicialab/avclass
} 


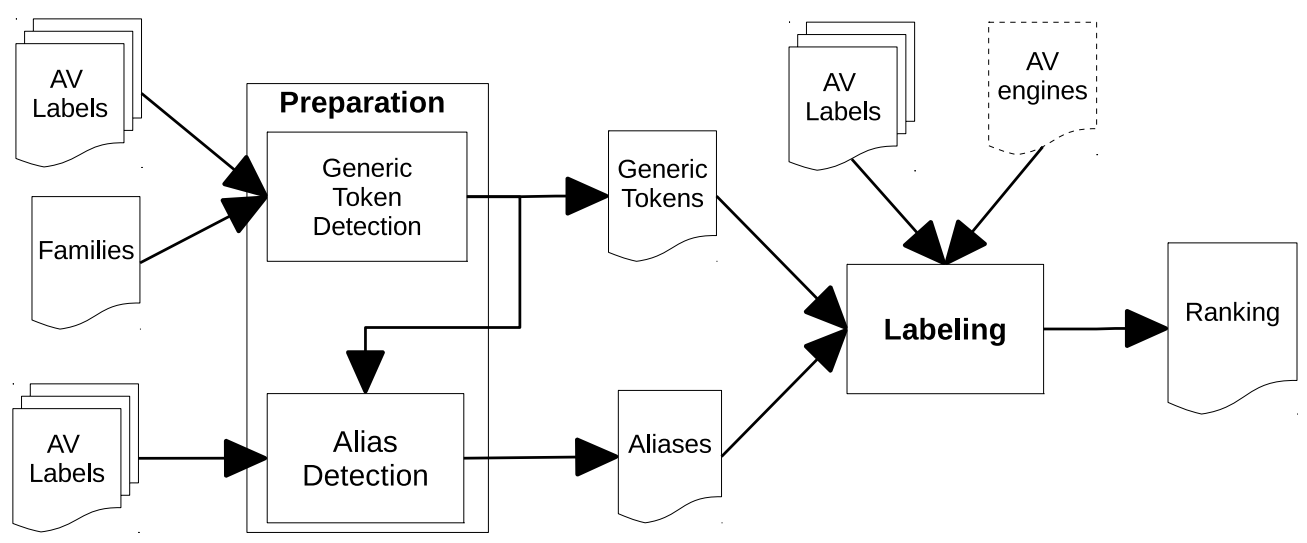

Figure 5.1: AVCLASS architecture.

list of generic tokens and aliases are the outputs of the preparation phase. By default, AVCLASS uses all AV engines in the set of AV labels for a sample. However, by providing an $\mathrm{AV}$ engine list, the analyst can restrict the processing to labels from those engines.

AVCLASS implements both the preparation and labeling phases. But, we expect that many analysts may not have large numbers of samples used for preparation. Thus, AVCLASS also includes default lists of generic tokens and aliases obtained in this work, so that an analyst can skip the preparation phase.

The remainder of this section first details the labeling phase (Section 5.2.1), and then the generic token detection (Section 5.2.2) and alias detection (Section 5.2.3) preparation modules. To illustrate the approach, we use the running example in Figure 5.2.

\subsubsection{Labeling}

The labeling phase takes as input the AV labels of a, potentially massive, number of samples. For each sample, it returns a ranking of its most likely family names. This Section describes the 8 steps used in labeling each sample.

AV selection (optional). By default, AVCLASS processes the labels of all AV engines in the input set of a sample. The labels of each sample may come from a different set of AV engines. This design decision was taken because selecting a fixed set of $\mathrm{AV}$ engines (as done in prior work) is difficult since there is no real information about which engines are better, and some engines may be good in a family but poor with others. Furthermore, a fixed set of AV engines throws away information as certain $\mathrm{AV}$ vendors may have been analyzed only by certain AV vendors. In particular, it is common to obtain AV labels from VT, which has used different AV engines to scan uploaded files over time. Overall, we observe 99 different AV engines in our VT reports, which are detailed in Table 5.1. Some engines are only seen for a few days, while others have been continually used by VT for nearly a decade. 


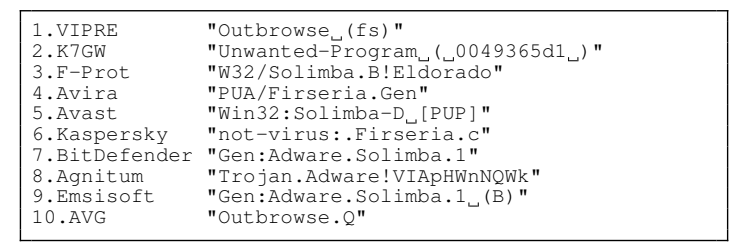

(a) AV labels

\begin{tabular}{|ll|}
\hline 1.VIPRE & "Outbrowse","fs" \\
2.K7GW & "Unwanted", "Program", "0049365d1" \\
3.F-Prot & "W32", "Solimba","B", "Eldorado" \\
4.Avira & "PUA", "Firseria" \\
5.Avast & "Win32",,"Solimba", "D", "PUP" \\
6.Kaspersky & "not","virus","MSIL","Firseria" \\
7.BitDefender & "Gen", "Adware","Solimba", "1" \\
8.Agnitum & "Trojan", "Adware" \\
10.AVG & "Outbrowse" \\
\hline
\end{tabular}

(c) After suffix removal and tokenization

\begin{tabular}{|ll|}
\hline 1.VIPRE & "outbrowse" \\
2.K7GW & "004965ad1" \\
3.F-Prot & "firseria" \\
4.Avira & "firseria" \\
5.Avast & "firseria" \\
6. Kaspersky & "firseria" \\
7. BitDefender & "firseria" \\
10.AVG & "outbrowse" \\
\hline
\end{tabular}

(e) After alias detection

\begin{tabular}{|c|c|}
\hline $\begin{array}{l}\text { 1.VIPRE } \\
\text { 2.K7GW } \\
\text { 3.F-Prot } \\
\text { 4.Avira } \\
\text { 5.Avast } \\
\text { 6. Kaspersky } \\
\text { 7. BitDefender } \\
\text { 8.Agnitum } \\
\text { 10.AVG }\end{array}$ & $\begin{array}{l}\text { "Outbrowse (fs)" } \\
\text { "Unwanted-Program (_0049365d1_) " } \\
\text { "W32/Solimba.B!Eldorado" } \\
\text { "PUA/Firseria.Gen" } \\
\text { "Win32:Solimba-D.[PUP] " } \\
\text { "not-virus:MSIL.Firseria.c" } \\
\text { "Gen:Adware.Solimba.1" } \\
\text { "Trojan.Adware!VIApHWnNQWk" } \\
\text { "Outbrowse.Q" }\end{array}$ \\
\hline
\end{tabular}

(b) After duplicate removal

\begin{tabular}{|ll|}
\hline 1.VIPRE & "outbrowse" \\
2.K7GW & "0049365d1" \\
3.F-Prot & "solimba" \\
4.Avira & "firseria" \\
5.Avast & "solimba" \\
6.Kaspersky & "firseria" \\
7.BitDefender & "solimba" \\
10.AVG & "outbrowse" \\
& \\
\hline
\end{tabular}

(d) After token filtering

\begin{tabular}{|ll|}
\hline $\begin{array}{ll}\text { 1.firseria } \\
\text { 2. outbrowse }\end{array}$ & 5 \\
\hline
\end{tabular}

(f) After token ranking

Figure 5.2: Running example.

Still, an analyst can optionally provide an input list of engines to AVCLASS. If provided, labels from engines not in the input list are removed at this step and only labels from the input set of AV engines are used for every sample. For brevity, our running example in Figure 5.2 assumes the analyst provided an input list with 10 engines. Figure 5.2a shows the input AV labels from the selected 10 engines for the same sample.

Duplicate removal. The same AV vendor may have multiple engines such as McAffee and McAffee-GW-Edition, or TrendMicro and TrendMicro-HouseCall. Those engines often copy labels from each other. While we could include only one engine per vendor, the reality is that their detections often differ. In addition, we observe groups of AV vendors that copy labels from each other, something also observed in prior work [29]. In both situations, the detection from these groups of engines are not independent (an assumption of some prior works [75]).

To avoid giving too much weight on the selected family name to vendors with multiple engines, or whose labels are often copied, we leverage the observation that when two AV engines output exactly the same label for a sample this very likely corresponds to one of those two situations. This happens because each vendor structures its labels differently and also uses slightly different keywords in their labels, so that two engines producing exactly the same label is rare unless they are copying each other. Thus, at this step, AVCLASS remove all duplicate labels. A special case is a vendor (Emsisoft) that when copying labels adds to them the suffix " (B)". For this vendor, we first remove this suffix from its labels, and then check for duplicates. We have not observed 
Table 5.1: AV engines found in our datasets and their lifetime in days.

\begin{tabular}{|c|c|c|c|}
\hline Engine & First Scan & Last Scan & Days \\
\hline Ikarus & $22 / 05 / 2006$ & $29 / 03 / 2016$ & 3599 \\
\hline TheHacker & $22 / 05 / 2006$ & $29 / 03 / 2016$ & 3599 \\
\hline F-Prot & $22 / 05 / 2006$ & $29 / 03 / 2016$ & 3599 \\
\hline Fortinet & $22 / 05 / 2006$ & $29 / 03 / 2016$ & 3599 \\
\hline BitDefender & $22 / 05 / 2006$ & $29 / 03 / 2016$ & 3599 \\
\hline CAT-QuickHeal & $22 / 05 / 2006$ & $29 / 03 / 2016$ & 3599 \\
\hline AVG & $22 / 05 / 2006$ & $29 / 03 / 2016$ & 3599 \\
\hline Microsoft & $22 / 05 / 2006$ & $29 / 03 / 2016$ & 3599 \\
\hline ClamAV & $22 / 05 / 2006$ & 29/03/2016 & 3599 \\
\hline Avast & $22 / 05 / 2006$ & 29/03/2016 & 3599 \\
\hline McAfee & $22 / 05 / 2006$ & $29 / 03 / 2016$ & 3599 \\
\hline TrendMicro & $22 / 05 / 2006$ & $29 / 03 / 2016$ & 3599 \\
\hline VBA32 & $22 / 05 / 2006$ & $29 / 03 / 2016$ & 3599 \\
\hline Symantec & $22 / 05 / 2006$ & $29 / 03 / 2016$ & 3599 \\
\hline Kaspersky & $22 / 05 / 2006$ & $29 / 03 / 2016$ & 3599 \\
\hline Panda & $22 / 05 / 2006$ & $29 / 03 / 2016$ & 3599 \\
\hline DrWeb & $22 / 05 / 2006$ & $29 / 03 / 2016$ & 3599 \\
\hline Sophos & $22 / 05 / 2006$ & $29 / 03 / 2016$ & 3599 \\
\hline F-Secure & $07 / 02 / 2007$ & $29 / 03 / 2016$ & 3338 \\
\hline AhnLab-V3 & $14 / 03 / 2007$ & $29 / 03 / 2016$ & 3303 \\
\hline Norman & $22 / 05 / 2006$ & $30 / 05 / 2015$ & 3294 \\
\hline Rising & 26/07/2007 & $29 / 03 / 2016$ & 3169 \\
\hline AntiVir & $22 / 05 / 2006$ & 03/09/2014 & 3025 \\
\hline GData & $12 / 05 / 2008$ & $29 / 03 / 2016$ & 2878 \\
\hline ViRobot & $24 / 07 / 2008$ & $29 / 03 / 2016$ & 2805 \\
\hline K7AntiVirus & 01/08/2008 & $29 / 03 / 2016$ & 2797 \\
\hline Comodo & $05 / 12 / 2008$ & $29 / 03 / 2016$ & 2671 \\
\hline nProtect & $14 / 01 / 2009$ & $29 / 03 / 2016$ & 2631 \\
\hline McAfee-GW-Edition & $19 / 03 / 2009$ & $29 / 03 / 2016$ & 2567 \\
\hline Antiy-AVL & 24/03/2009 & $29 / 03 / 2016$ & 2562 \\
\hline eSafe & $16 / 11 / 2006$ & $16 / 09 / 2013$ & 2496 \\
\hline Jiangmin & $16 / 06 / 2009$ & 29/03/2016 & 2478 \\
\hline VirusBuster & $13 / 06 / 2006$ & $18 / 09 / 2012$ & 2288 \\
\hline eTrust-Vet & $22 / 05 / 2006$ & $22 / 05 / 2012$ & 2191 \\
\hline TrendMicro-HouseCall & $04 / 05 / 2010$ & $29 / 03 / 2016$ & 2156 \\
\hline SUPERAntiSpyware & $12 / 07 / 2010$ & $29 / 03 / 2016$ & 2087 \\
\hline Emsisoft & $20 / 07 / 2010$ & $29 / 03 / 2016$ & 2079 \\
\hline VIPRE & $17 / 11 / 2010$ & 29/03/2016 & 1959 \\
\hline PCTools & $21 / 07 / 2008$ & $23 / 10 / 2013$ & 1919 \\
\hline Authentium & $22 / 05 / 2006$ & $29 / 04 / 2011$ & 1803 \\
\hline ByteHero & $20 / 08 / 2011$ & 29/03/2016 & 1683 \\
\hline Sunbelt & $30 / 11 / 2006$ & $29 / 04 / 2011$ & 1611 \\
\hline TotalDefense & $15 / 05 / 2012$ & $29 / 03 / 2016$ & 1414 \\
\hline NOD32 & $24 / 09 / 2008$ & $19 / 07 / 2012$ & 1394 \\
\hline ESET-NOD32 & $11 / 07 / 2012$ & $29 / 03 / 2016$ & 1357 \\
\hline Commtouch & $18 / 01 / 2011$ & $28 / 08 / 2014$ & 1317 \\
\hline Agnitum & $18 / 09 / 2012$ & $29 / 03 / 2016$ & 1288 \\
\hline Kingsoft & $18 / 09 / 2012$ & $29 / 03 / 2016$ & 1288 \\
\hline MicroWorld-eScan & $02 / 10 / 2012$ & $29 / 03 / 2016$ & 1274 \\
\hline NANO-Antivirus & $28 / 11 / 2012$ & $29 / 03 / 2016$ & 1217 \\
\hline
\end{tabular}

\begin{tabular}{|c|c|c|c|}
\hline Engine & First Scan & Last Scan & Days \\
\hline Malwarebytes & $30 / 11 / 2012$ & 29/03/2016 & 1215 \\
\hline $\mathrm{K} 7 \mathrm{GW}$ & $15 / 04 / 2013$ & 29/03/2016 & 1079 \\
\hline Prevx & $13 / 05 / 2009$ & 23/04/2012 & 1076 \\
\hline NOD32v2 & $22 / 05 / 2006$ & 19/01/2009 & 973 \\
\hline Ewido & $22 / 05 / 2006$ & 20/01/2009 & 973 \\
\hline eTrust-InoculateIT & $22 / 05 / 2006$ & $15 / 01 / 2009$ & 968 \\
\hline UNA & $22 / 05 / 2006$ & $15 / 01 / 2009$ & 968 \\
\hline Baidu & 02/09/2013 & 29/03/2016 & 939 \\
\hline Baidu-International & 03/09/2013 & 29/03/2016 & 938 \\
\hline F-Prot4 & $30 / 06 / 2006$ & $15 / 01 / 2009$ & 929 \\
\hline Bkav & $13 / 09 / 2013$ & 29/03/2016 & 928 \\
\hline Antivir7 & $22 / 06 / 2006$ & 05/01/2009 & 928 \\
\hline $\mathrm{CMC}$ & $13 / 09 / 2013$ & $29 / 03 / 2016$ & 928 \\
\hline $\mathrm{T} 3$ & $14 / 07 / 2006$ & $15 / 01 / 2009$ & 915 \\
\hline Prevx 1 & $15 / 11 / 2006$ & $12 / 05 / 2009$ & 909 \\
\hline Ad-Aware & $26 / 11 / 2013$ & $29 / 03 / 2016$ & 854 \\
\hline SAVMail & 03/10/2006 & $18 / 01 / 2009$ & 838 \\
\hline Qihoo-360 & $21 / 01 / 2014$ & 29/03/2016 & 798 \\
\hline AegisLab & 29/01/2014 & 29/03/2016 & 790 \\
\hline McAfee+Artemis & $21 / 11 / 2008$ & 18/01/2011 & 787 \\
\hline PandaBeta & $12 / 02 / 2007$ & $10 / 02 / 2009$ & 729 \\
\hline Zillya & 29/04/2014 & 29/03/2016 & 700 \\
\hline FileAdvisor & 19/02/2007 & $18 / 01 / 2009$ & 699 \\
\hline Tencent & $13 / 05 / 2014$ & $29 / 03 / 2016$ & 686 \\
\hline Zoner & $22 / 05 / 2014$ & 29/03/2016 & 677 \\
\hline Cyren & $22 / 05 / 2014$ & $29 / 03 / 2016$ & 677 \\
\hline Avira & $22 / 05 / 2014$ & 29/03/2016 & 677 \\
\hline Webwasher-Gateway & 20/03/2007 & $19 / 01 / 2009$ & 671 \\
\hline AVware & 28/07/2014 & 29/03/2016 & 610 \\
\hline a-squared & $24 / 12 / 2008$ & 28/07/2010 & 581 \\
\hline Avast5 & 03/03/2010 & 28/09/2011 & 573 \\
\hline McAfeeBeta & 04/07/2007 & $18 / 01 / 2009$ & 564 \\
\hline FortinetBeta & 01/08/2007 & 18/01/2009 & 535 \\
\hline PandaBeta2 & 07/09/2007 & $16 / 01 / 2009$ & 496 \\
\hline ALYac & $26 / 11 / 2014$ & $29 / 03 / 2016$ & 489 \\
\hline AhnLab & $14 / 03 / 2007$ & 03/07/2008 & 477 \\
\hline Alibaba & $12 / 01 / 2015$ & 29/03/2016 & 442 \\
\hline NOD32Beta & 24/09/2008 & $16 / 08 / 2009$ & 325 \\
\hline Arcabit & 02/06/2015 & 29/03/2016 & 301 \\
\hline SecureWeb-Gateway & 26/09/2008 & $14 / 04 / 2009$ & 200 \\
\hline VIRobot & $23 / 07 / 2008$ & $17 / 01 / 2009$ & 177 \\
\hline Command & $17 / 11 / 2010$ & 29/04/2011 & 163 \\
\hline PandaB3 & 04/09/2008 & $19 / 01 / 2009$ & 136 \\
\hline eScan & 25/09/2012 & $15 / 10 / 2012$ & 19 \\
\hline DrWebSE & $18 / 01 / 2015$ & $03 / 02 / 2015$ & 15 \\
\hline ESET NOD32 & $26 / 06 / 2012$ & 26/06/2012 & 0 \\
\hline Yandex & $29 / 03 / 2016$ & 29/03/2016 & 0 \\
\hline TotalDefense 2 & $16 / 04 / 2015$ & $16 / 04 / 2015$ & 0 \\
\hline SymCloud & $11 / 08 / 2015$ & $11 / 08 / 2015$ & 0 \\
\hline
\end{tabular}

any other such cases. Figure 5.2b shows how the Emsisoft label is removed at this step as a duplicate of the BitDefender label.

Suffix removal. We have empirically observed that most noise in AV labels is introduced in the suffix, i.e., the part of the AV label after the family name, where AV vendors may encode information such as rule numbers and hexadecimal strings that 
may be hashes. In general, it is difficult to remove those suffixes for all engines as vendors use different label structures, which may even change over time. Still, we have found 3 simple rules to truncate useless suffixes: (1) for $17 \mathrm{AV}$ engines, truncate label after last dot; (2) for $A V G$, truncate after last dot if the suffix only contains digits or uppercase chars; and (3) for Agnitum, truncate after the last '!' character. Suffix removal is the only engine-specific step in AVCLASS.

Tokenization. The next step is to split each label into tokens. We use a simple tokenization rule that splits the label on any sequence of consecutive non-alphanumeric characters. Figure 5.2c shows the results of the suffix removal and tokenization steps. Labels $4,6,8$, and 10 have been truncated by the suffix removal rules, and all labels have been tokenized.

Token filtering. The goal of this step is to remove tokens that are not family names. Each token goes through five substeps: (1) convert to lowercase; (2) remove digits at the end of the token; (3) remove token if short, i.e., less than 4 characters; (4) remove token if present in the input list of generic tokens; and (5) remove token if it is a prefix of the sample's hash ${ }^{2}$. Figure $5.2 \mathrm{~d}$ shows the results of token filtering where label 8 was removed as a result of not having any tokens left.

Alias replacement. Different vendors may use different names for the same family, i.e., aliases. If a token shows in the input list of aliases as being an alias for another family name, the token is replaced by the family name it aliases. The alias detection process is detailed in Section 5.2.3. Figure 5.2d shows the results after alias replacement, where token solimba has been identified as an alias for the firseria family.

Token ranking. Next, tokens are ranked by decreasing number of engines that include the token in their label. Tokens that appear in at most one AV engine are removed. This allows removing random tokens that earlier steps may have missed, as the likelihood is low that a random token appears in labels from multiple AV engines that did not copy their labels. At this point, the ranking captures the candidate family names for the sample and the number of AV engines that use each token can be seen as a confidence score. Figure 5.2f shows the final token ranking for our running example where token $0049365 \mathrm{~d} 1$ have been removed because it appears in only one label.

Family selection. AVCLASS chooses the most common token (top of the ranking) as the family name for the sample. This corresponds to a plurality vote on the candidate family names. AVCLASS also has a verbose option to output the complete ranking, which is useful to identify samples with multiple candidate family names with close scores, which may deserve detailed attention by the analyst. In our running example, the selected family is firseria, which outscores 5 to 2 the other possible family name.

\footnotetext{
${ }^{2}$ We check the sample's MD5, SHA1, and SHA256 hashes.
} 
Table 5.2: Categories in the manual generic token list.

\begin{tabular}{|l|r|l|}
\hline Category & Tokens & Example Tokens \\
\hline Architecture & 14 & android, linux, unix \\
Behavior: download & 29 & download, downware, dropped \\
Behavior: homepage modification & 2 & homepage, startpage \\
Behavior: injection & 5 & inject, injected, injector \\
Behavior: kill & 5 & antifw, avkill, blocker \\
Behavior: signed & 2 & fakems, signed \\
Behavior: other & 3 & autorun, proxy, servstart \\
Corrupted & 2 & corrupt, damaged \\
Exploit & 2 & expl, exploit \\
File types & 15 & html, text, script \\
Generic families & 13 & agent, artemis, eldorado \\
Heuristic detection & 12 & generic, genmalicius, heuristic \\
Macro & 11 & badmacro, macro, x2km \\
Malicious software & 5 & malagent, malicious, malware \\
Malware classes & 53 & spyware, trojan, virus \\
Misc & 9 & access, hack, password \\
Packed & 17 & malpack, obfuscated, packed \\
Packer & 6 & cryptor, encoder, obfuscator \\
Patch & 3 & patched, patchfile, pepatch \\
Program & 5 & application, program, software \\
PUP & 29 & adware, pup, unwanted \\
Suspicious & 13 & suspected, suspicious, variant \\
Test & 2 & test, testvirus \\
Tools & 8 & fraudtool, tool, virtool \\
Unclassified & 3 & unclassifiedmalware, undef, unknown \\
\hline
\end{tabular}

\subsubsection{Generic Token Detection}

AV labels typically contain multiple generic tokens not specific to a family. For example, the labels in Figure 5.2 include generic tokens indicating, among others, the sample's architecture (e.g., Win32, Android), that a sample is unwanted (e.g., Unwanted, Adware, PUP), generic malware classes (e.g., Trojan), and generic families used with heuristic rules (e.g., Eldorado, Artemis). The generic token detection module is used during the preparation phase to automatically build a list of generic tokens used as input to the labeling phase in Section 5.2.1.

The intuition behind our technique for identifying generic tokens is that tokens appearing in the labels of samples known to be of different families cannot be specific to a family, and thus are generic. For example, an AV engine may output the label Gen:Adware.Firseria.1 for a sample known to be of the Firseria adware family and the label Gen:Adware.Outbrowse. 2 for a sample known to be of the Outbrowse adware family. Here, tokens Gen and Adware are likely generic because they are used with samples of different families, and thus are not specific to the Firseria or Outbrowse families. 
The generic token detection module takes as input samples for which their family name is known. It iterates on the list of input samples. For each sample, it builds a sample token list, by iterating on the set of AV labels for the sample. For each label, it tokenizes the label on non-alphanumeric characters, converts tokens to lowercase, removes digits at the end of the token, removes tokens less than 4 characters, and appends the remaining tokens to the sample token list. Once all labels are processed, it removes duplicate tokens from the sample token list. The sample token list for the sample in Figure 5.2 would be: outbrowse, unwanted, program, 0049365d1, solimba, eldorado, firseria, virus, msil, adware, and trojan. Then, it iterates on the tokens in the sample token list updating a token family map, which maps each unique token to the list of families of the samples where the token appears in their labels.

After all samples have been processed, it iterates on the token family map. Each token that does not match a family name and has a count larger than $T_{g e n}$ is considered generic. The default $T_{\text {gen }}>8$ threshold is chosen empirically in Section 5.3.3. For example, tokens firseria and solimba may have appeared only in labels of samples from the Firseria family and thus are not generic, but token eldorado may have appeared in labels from samples of 9 different families and is identified as generic.

We have applied this approach to automatically generate a list of generic tokens. One author has also manually generated a list of generic tokens. Our experiments in Section 5.3.3 show that the automatically generated generic token list performs similarly in most cases, and even outperforms the manually generated lists in some cases, while scaling and being independent of an analyst's expertise.

Table 5.2 shows the 15 categories of generic tokens in the manually built generic token list. For each category, it shows the number of tokens in the category and some example tokens. The categories show the wealth of information that AV vendors encode in their labels. They include, among others, architectures; behaviors like homepage modification, code injection, downloading, and disabling security measures; file types; heuristic detections; macro types; malware classes; encrypted code; and keywords for potentially unwanted programs. The categories with more generic tokens are malware classes with 53 tokens (e.g., trojan, virus, worm, spyware), download behavior with 29 (e.g., download, dload, downl, downware), and potentially unwanted programs with 29 (e.g., pup, adware, unwanted).

\subsubsection{Alias Detection}

Different vendors may assign different names (i.e., aliases) for the same family. For example, some vendors may use zeus and others zbot as aliases for the same malware family. The alias detection module is used during the preparation phase to automatically build a list of aliases used as input to the labeling phase in Section 5.2.1.

The intuition behind our technique for automatically detecting aliases is that if two family names are aliases, they will consistently appear in the labels of the same samples. Alias detection takes as input the AV labels of a large set of samples, for which their family does not need to be known, and a generic token list. Thus, alias 
Table 5.3: Top 10 families by number of aliases.

\begin{tabular}{|l|r|l|}
\hline Family & Aliases & Example Aliases \\
\hline wapomi & 12 & pikor, otwycal, protil \\
firseria & 10 & firser, popeler, solimba \\
vobfus & 9 & changeup, meredrop, vbobfus \\
virut & 8 & angryangel, madangel, virtob \\
gamarue & 7 & debris, lilu, wauchos \\
hotbar & 7 & clickpotato, rugo, zango \\
bandoo & 6 & ilivid, seasuite, searchsuite, \\
gamevance & 6 & arcadeweb, gvance, rivalgame \\
loadmoney & 6 & ldmon, odyssey, plocust \\
zeroaccess & 6 & maxplus, sirefef, zaccess \\
\hline
\end{tabular}

detection runs after the generic token detection, which prevents generic tokens to be detected as aliases. Alias detection outputs a list of $\left(t_{i}, t_{j}\right)$ token pairs where $t_{i}$ is an alias for $t_{j}$. This indicates that $t_{i}$ can be replaced with $t_{j}$ in the alias detection step in Section 5.2.1.

Alias detection iterates on the list of input samples. For each sample, it builds the sample token list in the same manner as described in the generic token detection in Section 5.2.2, except that tokens in the generic token list are also removed. Then, it iterates on the tokens in the sample token list updating two maps. It first increases the token count map, which stores for each unique token the number of samples where the token has been observed in at least one label. Then, for each pair of tokens in the sample token list it increases the pair count map that stores for each token pair the number of samples in which those two tokens have been observed in their labels.

We define the function alias $\left(t_{i}, t_{j}\right)=\frac{\left|\left(t_{i}, t_{j}\right)\right|}{\left|t_{i}\right|}$, which captures the fraction of times that the pair of tokens $\left(t_{i}, t_{j}\right)$ appears in the same samples. The numerator can be obtained from the pair count map and the denominator from the token count map. Note that $\operatorname{alias}\left(t_{i}, t_{j}\right) \neq \operatorname{alias}\left(t_{j}, t_{i}\right)$.

Once all samples have been processed, the alias detection iterates on the pair count map. For each pair that has a count larger than $n_{\text {alias }}$, it computes both alias $\left(t_{i}, t_{j}\right)$ and alias $\left(t_{j}, t_{i}\right)$. If alias $\left(t_{i}, t_{j}\right)>T_{\text {alias }}$ then $t_{i}$ is an alias for $t_{j}$. If alias $\left(t_{j}, t_{i}\right)>T_{\text {alias }}$ then $t_{j}$ is an alias for $t_{i}$. If both alias $\left(t_{i}, t_{j}\right)>T_{\text {alias }}$ and alias $\left(t_{j}, t_{i}\right)>T_{\text {alias }}$ then the less common token is an alias for the most common one.

The two parameters are empirically selected in Section 5.3.4 to have default values $n_{\text {alias }}=20$ and $T_{\text {alias }}=0.94$. $n_{\text {alias }}$ is used to remove pairs of tokens that have not been seen enough times, so that a decision on whether they are aliases would have low confidence. $T_{\text {alias }}$ controls the percentage of times the two tokens appear together. For $t_{j}$ to be an alias for $t_{i}, t_{j}$ should appear in almost the same samples where $t_{i}$ appears, but $T_{\text {alias }}$ is less than one to account for naming errors.

Table 5.3 shows the Top 10 families by number of aliases. For each alias, it shows the chosen family name, the total number of aliases for that family, and some example 
Table 5.4: Datasets used in evaluation.

\begin{tabular}{|l|l|c|r|c|c|}
\hline Dataset & Platform & Lab. & Samples & EXE & Collection Period \\
\hline University & Windows & - & $7,252,810$ & - & $01 / 2011-08 / 2015$ \\
Miller et al. [76] & Windows & - & $1,079,783$ & - & $01 / 2012-06 / 2014$ \\
Andrubis [138] & Android & - & 422,826 & - & $06 / 2012-06 / 2014$ \\
Malsign [2] & Windows & $\checkmark$ & 142,513 & - & $06 / 2012-02 / 2015$ \\
VirusShare_20140324 [139] & Android & - & 24,317 & $\checkmark$ & $05 / 2013-05 / 2014$ \\
VirusShare_20130506 [139] & Android & - & 11,080 & $\checkmark$ & $06 / 2012-05 / 2013$ \\
Malicia [137] & Windows & $\checkmark$ & 9,908 & $\checkmark$ & $03 / 2012-02 / 2013$ \\
Drebin [74] & Android & $\checkmark$ & 5,560 & $\checkmark$ & $08 / 2010-10 / 2012$ \\
Malheur Reference [69] & Windows & $\checkmark$ & 3,131 & - & $08 / 2009-08 / 2009$ \\
MalGenome [136] & Android & $\checkmark$ & 1,260 & $\checkmark$ & $08 / 2008-10 / 2010$ \\
\hline
\end{tabular}

aliases that appear both in the automatically and manually generated alias lists.

\subsection{Evaluation}

\subsubsection{Datasets}

We evaluate AVCLASS using 10 datasets summarized in Table 5.4. The table shows the architecture (5 Windows, 5 Android), whether the samples are labeled with their known family name, the number of samples in the dataset, whether the binaries are publicly available (otherwise we only have their hashes), and the collection period. In total, the datasets contain 8.9 M distinct samples collected during 7 years (08/2008 - 08/2015). Some of the datasets overlap, most notably the Drebin [74] dataset is a superset of MalGenome [136]. We do not remove duplicate samples because this way it is easier for readers to map our results to publicly available datasets.

Labeled datasets. All 5 labeled datasets come from prior works [2,69,74, 136, 137]. Among the 3 labeled Windows datasets, Malheur and Malicia contain only malware samples. In contrast, the Malsign dataset [2] contains majoritarily PUP. Each of the labeled datasets went through 2 processes: clustering and labeling. Samples may have been clustered manually (MalGenome), using AV labels (Drebin), or with automatic approaches (Malheur, Malicia, Malsign). For labeling the output clusters, the authors may have used AV labels (Drebin), manual work (MalGenome), the most common feature value in the cluster (Malsign), or a combination of popular features values and information from public sources (Malicia). Drebin [74] is a detection approach and the family classification was done separately using AV labels. Because of this we later observe best results of AVCLASS on this dataset. able Drebin, MalGenome, Malheur, and Malicia datasets are publicly available. Thus, AV vendors could have refined their detection labels using the dataset clustering results after they became public. In contrast, the Malsign dataset and thus its clustering results (i.e., labels) are not publicly 
available.

Unlabeled datasets. For the unlabeled datasets, we do not know the family of the samples and in some cases we only have access to the hashes of the samples, but not their binaries. The University dataset contains malware hashes collected from different sources including a commercial feed. It is our largest dataset with 7.2 M samples. The Andrubis dataset [138] contains hashes of samples submitted by users to be analyzed by the Andrubis online service. The two VirusShare [139] and the Miller et al. [76] datasets are publicly available.

For all samples in the 10 datasets we were able to collect a VT report. The VT report collection started on September 2015 and took several months. Overall, we observe $99 \mathrm{AV}$ engines in the VT reports.

\subsubsection{Metrics}

To evaluate the accuracy of AVCLASS, we use an external clustering validation approach that compares AVCLASS's clustering results with a reference clustering from one of the datasets in Table 5.4 for which we have ground truth. Note that the external validation evaluates if both clusterings group the samples similarly. It does not matter if the family names assigned to the equivalent cluster in both clustering differ. If AVCLASS is not able to find a family name for a sample (e.g., because all its labels are generic), the sample is placed in a singleton cluster. Similar to prior work $[2,67,69,72,137]$ we use the precision, recall, and F1 measure metrics, which we define next.

Let $M$ be a malware dataset, $R=\left\{R_{1}, \ldots, R_{s}\right\}$ be the set of $s$ reference clusters from the dataset's ground truth, and $C=\left\{C_{1}, \ldots, C_{n}\right\}$ be the set of $n$ clusters output by AVCLASS over $M$. In this setting, precision, recall, and F1 measure are defined as

- Precision. Prec $=1 / n \cdot \sum_{j=1}^{n} \max _{k=1, \ldots, s}\left(\left|C_{j} \bigcap R_{k}\right|\right)$

- Recall. $R e c=1 / s \sum_{k=1}^{s} \max _{j=1, \ldots, n}\left(\left|C_{j} \bigcap R_{k}\right|\right)$

- F-measure Index. $F 1=2 \frac{\text { Prec } \cdot \text { Rec }}{\text { Prec }+ \text { Rec }}$

\subsubsection{Generic Token Detection}

The generic token detection, detailed in Section 5.2.2, takes as input the AV labels for samples with family name and counts the number of families associated to each remaining token after normalization. Tokens that appear in more than $T_{\text {gen }}$ families are considered generic. To select the default threshold, we produce generic token lists for different $T_{\text {gen }}$ values and evaluate the accuracy of the labeling phase using those generic token lists. Figure 5.3 shows the $\mathrm{F} 1$ measure as $T_{\text {gen }}$ increases for datasets with ground truth. Based on Figure 5.3 results, we select $T_{g e n}>8$ as the default 


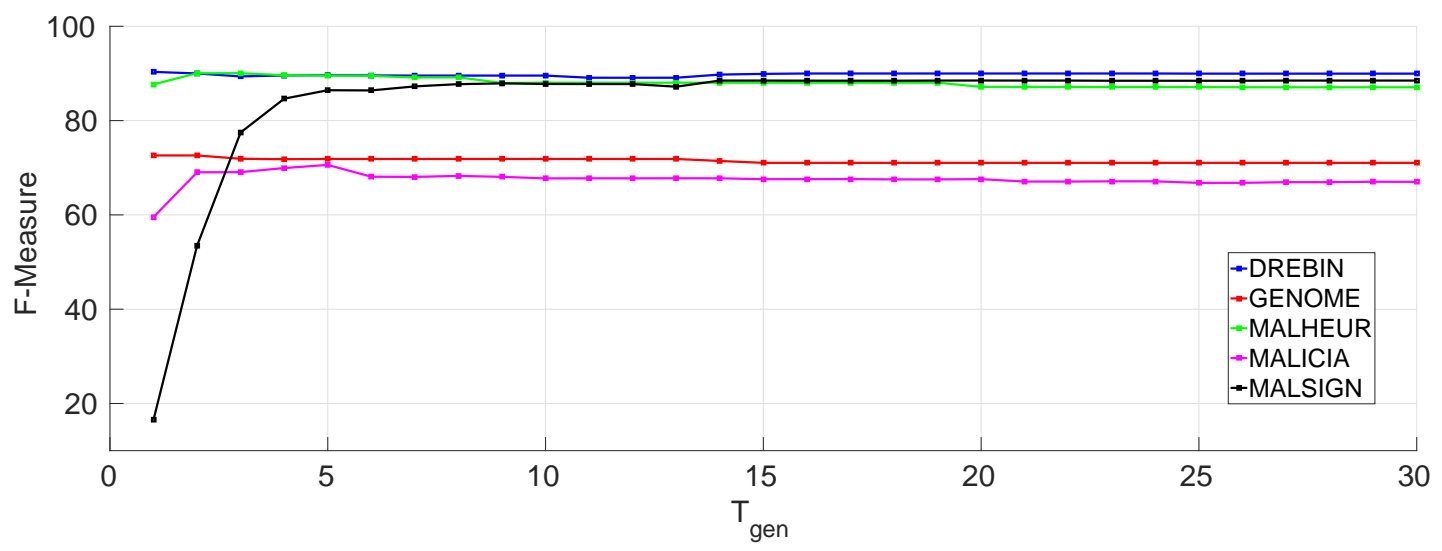

Figure 5.3: Parameter selection for generic token detection.

threshold. The rest of experiments use, unless otherwise noted, the automatically generated generic token list with this default threshold, which contains 288 generic tokens. In comparison the generic token list manually generated by one author contains 240 generic tokens.

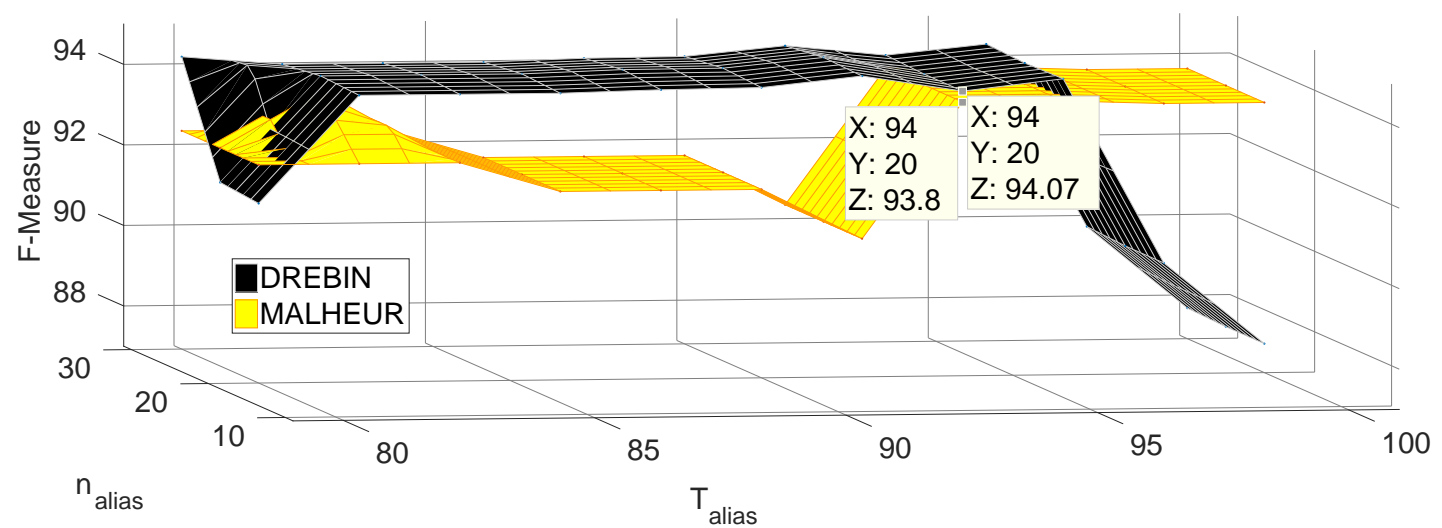

Figure 5.4: Parameter selection for alias detection.

\subsubsection{Alias Detection}

The alias detection module, detailed in Section 5.2.3, requires two parameters: $n_{\text {alias }}$ and $T_{\text {alias }}$. To select their default values, we first produce alias lists for different combinations of those parameters using as input the 5 datasets with unlabeled samples. Then, we evaluate the accuracy of the labeling phase using those alias lists. Figure 5.4 shows the $F_{1}$ measure for different combinations of parameter values on the Drebin and Malheur datasets. The parameter values that maximize the mean value in both surfaces are $n_{\text {alias }}=20$ and $T_{\text {alias }}=0.94$. The rest of experiments use, unless otherwise noted, the automatically generated alias list with these default values, which contains 4,332 alias pairs. In comparison, the alias list manually generated by one author contains 133 alias pairs. 
Table 5.5: Accuracy evaluation. Full Label corresponds to using a plurality vote on all labels without normalization. Manual corresponds to running AVCLASS with manually generated generic token and alias lists. AVCLASS corresponds to running AVCLASS with automatically generated generic token and alias lists. The MalGenome* row corresponds to grouping the 6 DroidKungFu variants in MalGenome into a single family.

\begin{tabular}{|l|r|r|r||r|r|r||r|r|r|}
\cline { 2 - 10 } \multicolumn{1}{c|}{} & \multicolumn{3}{c||}{ AVCLASS } & \multicolumn{3}{c||}{ Manual } & \multicolumn{3}{c|}{ Full Label } \\
\hline Dataset & Prec & Rec & F1 & Prec & Rec & F1 & Prec & Rec & F1 \\
\hline Drebin & 95.2 & 92.5 & 93.9 & 95.4 & 88.4 & 91.8 & 92.9 & 40.7 & 56.6 \\
Malicia & 95.3 & 46.3 & 62.3 & 94.9 & 68.0 & 79.2 & 98.6 & 2.4 & 4.6 \\
Malsign & 96.3 & 67.0 & 79.0 & 90.4 & 90.7 & 90.5 & 88.7 & 15.9 & 26.9 \\
MalGenome & 67.5 & 98.8 & 80.2 & 68.3 & 93.3 & 78.8 & 99.5 & 79.4 & 88.3 \\
MalGenome* & 87.2 & 98.8 & 92.6 & 87.9 & 93.3 & 90.5 & 99.7 & 63.3 & 77.5 \\
Malheur & 89.3 & 93.8 & 91.5 & 90.4 & 98.3 & 94.2 & 96.3 & 74.8 & 84.2 \\
\hline
\end{tabular}

\subsubsection{Evaluation on Labeled Datasets}

In this section we evaluate the accuracy of AVCLASS on the labeled datasets. We first compare the reference clustering provided by the dataset labels with the clustering output by AVCLASS (i.e., samples assigned the same label by AVCLASS are in the same cluster) using the precision, recall, and F1 measure metrics introduced in Section 5.3.2. Then, we examine the quality of the output labels.

Clustering accuracy. Table 5.5 summarizes the clustering accuracy results for 3 scenarios. Full Label corresponds to not using AVCLASS but simply doing a plurality vote on the full AV labels without normalization. Manual corresponds to running AVCLASS with manually generated generic token and alias lists. AVCLASS corresponds to running AVCLASS with automatically generated generic token and alias lists.

The results show that using AVCLASS increases the F1 measure compared to using the full label in 4 datasets (Drebin, Malicia, Malsign, and Malheur). The median F1 measure improvement is $37 \mathrm{~F} 1$ measure percentual points and can reach 13 times higher (Malicia). The exception is the MalGenome dataset, whose F1 measure decreases. Manual examination shows that the main problem is that the MalGenome dataset differentiates 6 variants of the DroidKungFu family (DroidKungFu1, DroidKungFu2, DroidKungFu3, DroidKungFu4, DroidKungFuSapp, DroidKungFuUpdate). However, AV labels do not capture version granularity and label all versions as the same family. If we group all 6 DroidKungFu variants into a single family (MalGenome* row in Table 5.5), the F1 measure using AVCLASS increases 12 points (from 80.2 to 92.6) and the full label results decreases 11 points (from 88.3 to 77.5). This shows that AV labels are not granular enough to identify specific family versions.

Comparing the Manual section of Table 5.5 with the AVCLASS section shows that the automatically generated lists of generic tokens and aliases work better in 2 datasets 
Table 5.6: Labels for the top 5 clusters identified by AVCLASS in the Miller et al. dataset and the most common full labels on the same dataset.

(a) AVCLASS.

(b) Full labels.

\begin{tabular}{|r|l|r||l|l|r|}
\hline$\#$ & Label & Samples & $\#$ & Label & Samples \\
\hline 1 & vobfus & 58,385 & 1 & Trojan.Win32.Generic!BT & 42,944 \\
\hline 2 & domaiq & 38,648 & 2 & Win32.Worm.Allaple.Gen & 12,090 \\
\hline 3 & installrex & 37,698 & 3 & Gen:Variant.Adware.Graftor.30458 & 10,844 \\
\hline 4 & firseria & 28,898 & 4 & Gen:Adware.MPlug.1 & 10,332 \\
\hline 5 & multiplug & 26,927 & 5 & Trojan.Generic.6761191 & 8,986 \\
\hline
\end{tabular}

(MalGenome and Drebin) and worse in 3 (Malicia, Malsign, Malheur). For Malheur the difference is small (2.7 F1 points), but for Malicia and Malsign it reaches 11-17 F1 points. Overall, the automatically generated lists have comparable accuracy to the manual ones, although an analyst can improve results in some datasets. While the manual list raises the worst F1 measure from 62.3 to 79.2 , the automatic generation is faster, more convenient, and does not depend on the analyst's skill. To combine scalability with accuracy, an analyst could first produce automatically the lists and then refine them manually based on his expertise.

The final F1 measure for AVCLASS with automatically generated lists of generic tokens and aliases ranges from 93.9 for Drebin down to 62.3 for Malicia. The higher accuracy for Drebin is due to that dataset having been manually clustered using AV labels. The lower accuracy for Malicia is largely due to smartfortress likely being an (undetected) alias for the winwebsec family. Manually adding this alias improves the F1 measure by 18 points. The reason for the large impact of this alias is that the Malicia dataset is strongly biased towards this family (59\% of samples are in family winwebsec).

Label quality. The clustering evaluation above focuses on whether samples are grouped by AVCLASS similarly to the ground truth, but it does not evaluate the quality of the family names AVCLASS outputs. Quantifying the quality of the family names output by AVCLASS is challenging because the ground truth may contain manually selected labels that do not exactly match the AV family names. Table 5.6 shows on the left the labels assigned to the top 5 clusters in the Miller dataset by AVCLASS and on the right, the labels for the top 5 clusters when the full AV labels are used. The table shows that the cluster labels automatically produced by AVCLASS are more finegrained thanks to the generic token detection, and also assigned to a larger number of samples thanks to the normalization and alias detection techniques. More examples of the final labels output by AVCLASS are shown in Table 5.8, which is discussed in the next section. 
Table 5.7: Clustering results on unlabeled datasets.

\begin{tabular}{|l|r|r|r|r|r|r|}
\hline Dataset & Samples & Clusters & Singletons & Unlab. & Largest & Runtime \\
\hline University & $7,252,810$ & $1,465,901$ & $1,456,375$ & $19.2 \%$ & 701,775 & $235 \mathrm{~min} .33 \mathrm{~s}$ \\
\hline Miller et al. & $1,079,783$ & 187,733 & 183,804 & $16.6 \%$ & 56,044 & $35 \mathrm{~min} .42 \mathrm{~s}$ \\
\hline Andrubis & 422,826 & 7,015 & 6,294 & $1.3 \%$ & 102,176 & $12 \mathrm{~min} .47 \mathrm{~s}$ \\
\hline VirusShare_20140324 & 24,317 & 2,068 & 1,844 & $6.9 \%$ & 7,350 & $48 \mathrm{~s}$ \\
\hline VirusShare_20130506 & 11,080 & 568 & 446 & $3.3 \%$ & 3,203 & $17 \mathrm{~s}$ \\
\hline
\end{tabular}

\subsubsection{Evaluation on Unlabeled Datasets}

In this section we apply AVCLASS to label samples in datasets without ground truth. Table 5.7 summarizes the clustering results of using AVCLASS with automatically generated lists on the 5 unlabeled datasets. For each dataset it shows: the number of samples being clustered, the number of clusters created, the number of singleton clusters with only one sample, the percentage of all samples that did not get any label, the size of the largest cluster, and the labeling runtime. The results show that $78 \%-99 \%$ of the clusters are singletons. However, these only represent $1.4 \%-20 \%$ of the samples. Thus, the vast majority of samples are grouped with other samples. Singleton clusters can be samples for which no label can be extracted as well as samples assigned a label not seen in other samples. Overall, the percentage of unlabeled samples varies from $1.3 \%$ (Andrubis) up to 19.2\% (University). All AV labels for these samples are generic and AVCLASS could not identify a family name in them.

Table 5.8 presents the top 10 clusters in the 3 largest unlabeled datasets (University, Miller, Andrubis). The most common family in both Windows datasets is vobfus. Top families in these two datasets are well known except perhaps flooder, which the author building the manual lists thought it was generic, but the automatic generic token detection does not identify as such. This is an example of tokens that may sound generic to an analyst, but may be consistently used by AV vendors for the same family. In the University dataset 6 of the top 10 families are malware (vobfus, multiplug, virut, toggle, flooder, upatre) and 4 are PUP (softpulse, loadmoney, zango, ibryte). In the Miller dataset 3 are malware (vobfus, zbot, sality) and 7 PUP (firseria, installerex, domaiq, installcore, loadmoney, hotbar, ibryte). This matches observations in Malsign [2] that large "malware" datasets actually do contain significant amounts of PUP. The Andrubis top 10 contains 4 families that also sound generic (opfake, fakeinst, smsagent, smsreg). However, these families are included as such in the ground truth of the labeled Android datasets (MalGenome, Drebin). While these labels may be used specifically for a family, we believe AV vendors should try choosing more specific family names to avoid one vendor using a label for a family and another using it generically for a class of malware.

Number of AV vendors used. To evaluate the effect of using an increasing number of $\mathrm{AV}$ vendors into the labeling process, we repeat the clustering of the University dataset using the same fixed sets of AV vendors used in some prior work: VAMO (4 
Table 5.8: Top 10 clusters on unlabeled datasets.

(a) University.

\begin{tabular}{|r|l|r|}
\hline$\#$ & Label & Samples \\
\hline 1 & vobfus & 701,775 \\
\hline 2 & multiplug & 669,596 \\
\hline 3 & softpulse & 473,872 \\
\hline 4 & loadmoney & 211,056 \\
\hline 5 & virut & 206,526 \\
\hline 6 & toggle & 108,356 \\
\hline 7 & llooder & 96,571 \\
\hline 8 & zango & 89,929 \\
\hline 9 & upatre & 82,413 \\
\hline 10 & ibryte & 80,923 \\
\hline
\end{tabular}

(b) Miller.

\begin{tabular}{|r|l|r|}
\hline$\#$ & Label & Samples \\
\hline 1 & vobfus & 58,385 \\
\hline 2 & domaiq & 38,648 \\
\hline 3 & installrex & 37,698 \\
\hline 4 & firseria & 28,898 \\
\hline 5 & multiplug & 26,927 \\
\hline 6 & sality & 23,278 \\
\hline 7 & zango & 21,910 \\
\hline 8 & solimba & 21,305 \\
\hline 9 & ibryte & 20,058 \\
\hline 10 & expiro & 16,685 \\
\hline
\end{tabular}

(c) Andrubis.

\begin{tabular}{|r|l|r|}
\hline$\#$ & Label & Samples \\
\hline 1 & opfake & 88,723 \\
\hline 2 & fakeinst & 84,485 \\
\hline 3 & smsagent & 24,121 \\
\hline 4 & plankton & 22,329 \\
\hline 5 & kuguo & 19,497 \\
\hline 6 & smsreg & 15,965 \\
\hline 7 & waps & 12,055 \\
\hline 8 & utchi & 7,949 \\
\hline 9 & droidkungfu & 7,675 \\
\hline 10 & ginmaster & 6,365 \\
\hline
\end{tabular}

vendors), Drebin (10), and AV-meter (48). The results in Table 5.9 show that increasing the number of $\mathrm{AV}$ vendors reduces the fraction of samples for which a label cannot be obtained (Unlabeled column). This motivates the design choice of AVCLASS to include AV labels from any available vendor.

\subsection{Discussion}

This section discusses AVCLASs limitations, usage, and areas for future work.

As good as the AV labels are. AVCLASS extracts family information AV vendors place in their labels, despite noise in those labels. But, it cannot identify families not in the labels. More specifically, it cannot label samples if at least $2 \mathrm{AV}$ engines do not agree on a non-generic family name. Results on our largest unlabeled dataset show that AVCLASS cannot label $19 \%$ of the samples, typically because those labels only contain generic tokens. Thus, AVCLASS is not a panacea for malware labeling. If AV 
Table 5.9: University dataset clustering with 4, 10, 48, and all AVs.

\begin{tabular}{|l|r|r|r|l|}
\hline AVs & Clusters & Singletons & Unlabeled & Largest \\
\hline All & $1,465,901$ & $1,456,375$ & $1,394,168(19.2 \%)$ & vobfus $(701,775)$ \\
\hline 48 & $1,543,510$ & $1,534,483$ & $1,472,406(20.3 \%)$ & vobfus $(701,719)$ \\
\hline 10 & $3,732,626$ & $3,730,304$ & $3,728,945(51.4 \%)$ & multiplug $(637,787)$ \\
\hline 4 & $5,655,991$ & $5,655,243$ & $5,654,819(77.9 \%)$ & vobfus $(539,306)$ \\
\hline
\end{tabular}

vendors do not have a name for the sample, it cannot be named.

Table 5.10: Accuracy numbers reported by prior clustering works.

\begin{tabular}{|l|l|}
\hline Work & Metrics \\
\hline Bailey et al. [27] & Consistency=100\% \\
Rieck et al. [17] & Labels prediction=70\% \\
McBoost [66] & Accuracy=87.3\%, AUC=0.977. \\
Bayer et al. [67] & Quality(Prec*Rec)=95.9 \\
Malheur [69] & F1=95\% \\
BitShred [70] & Prec=94.2\%, Rec=92.2\% \\
VAMO [72] & F1=85.1\% \\
Malsign [2] & Prec=98.6\%, Rec=33.2\%, F1=49.7\% \\
\hline AVCLASS & Prec=95.2\%, Rec=92.5\%, F1=93.9\% \\
\hline
\end{tabular}

Clustering accuracy. AVCLASS is a malware labeling tool. While it can be used for malware clustering, its evaluated precision is $87.2 \%-95.3 \%$. This is below stateof-the-art malware clustering tools using static and dynamic features, which can reach 98\%-99\% precision. As shown in Table 5.10, when comparing F1 measure, tools like Malheur [69] (F1= 95\%), BitShred [70] (F1=93.2\%), and FIRMA [133] (F1=98.8\%) outperform AVCLASS. Thus, AVCLASS should only be used for clustering when a state-of-the-art clustering system is not available and implementing one is not worth the effort (despite improved accuracy).

Building reference datasets. When using AVCLASS to build reference datasets, there will be a fraction of samples (up to $19 \%$ in our evaluation) for which AVCLASS cannot extract a label and others for which the confidence (i.e., number of AV engines using the chosen family name) is low. While those can be removed from the reference dataset, this introduces selection bias by removing the harder to label samples [82].

AV label granularity. Our evaluation shows that AV labels are not granular enough to differentiate family versions, e.g., DroidKungFu1 from DroidKungFu2. Thus, when releasing labeled datasets, researchers should clearly differentiate the family name from the family version (if available), enabling users to decide which granularity to use. 
Validation with real ground truth. To evaluate AVCLASS, we have assumed that the labels of publicly available datasets are perfectly accurate and have compared accuracy to those. However, those labels may contain inaccuracies, which would affect our results either positively or negatively. This can only be resolved by using real ground truth datasets. How to obtain such real ground truth is an important area for future work.

Generic token detection. Our generic token detection requires labeled samples. This creates a bit of a chicken-and-egg problem, which we resolve by using publicly available labeled datasets. We also release a file with the generic tokens we identified so that users can skip this step. We leave the development of techniques to identify generic tokens that do not require ground truth for future work. 


\section{Conclusions}

PUP operates on the gray zone between malicious software (i.e., malware) and benign software. The boundaries between benign software and PUP, and between PUP and malware are oftentimes blurry. To address this issue security vendors have proposed guidelines and policies to delineate what constitutes PUP. But, those guidelines and policies have been designed for manual analysts. Currently, there are no automatic and effective techniques for PUP detection and analysis.

In this thesis we have proposed novel tools to detect PUP behaviors, and to analyze PUP from the points of view of its distribution and its classification. We have first presented a measurement study of the prevalence of PUP in download portals. We have built a platform to analyze the abuse in 20 download portals crawling a total of $191 \mathrm{~K}$ Windows freeware installers. In contrast with previous works that concluded that download portals are bloated with undesirable programs, our measurements show that the amount of potentially unwanted programs and malware in 18 of the 20 download portals is below 9\%. The other two download portals were used exclusively to distribute PPI downloaders, which means that their amount of malware and potentially unwanted programs is $100 \%$. Furthermore we describe several abusive behaviors that we observed in our analysis. The first one is that the same program file appears in different download portals, and even within the same download portals as a different program, or under different authors. Another behavior that we found is that some program authors impersonate popular benign authors in order to make their programs more attractive or make their undesirable programs looks benign. We also observe that authors that apply one or more of these abusive behaviors, they do it in multiple download portals.

Next, we have designed and implemented the first platform to automatically detect PUP behaviors. It receives executables to run in a sandbox. If the input executable is an installer, our platform analyzes the installation, detects PUP behaviors in the installation, identifies the list of installed programs, detects whether they have an uninstaller, tries to uninstall those programs, detects PUP behaviors in the uninstallation, and checks if the programs have been completely uninstalled. We have evaluated our 
platform using $16 \mathrm{~K}$ from download portals and 50 benign installers. We have developed some improvements to the GUI interaction of Cuckoo Sandbox to automate the installation and the uninstallation phases with programs that require human interaction through the GUI. Program analysis could benefit of those improvements, but GUI exploration presents several challenges, such as different character encoding used by the developers, and the variety of libraries used to implement the GUI of an application. Previous works [1] has tried to use image recognition to deal with the automation of GUI exploration, but they show that their approach still have limitations due to incorrect UI interactions. Addressing these problems is beyond of the scope of this thesis, and we leave it to future work.

Finally, we have described AVCLASS, an automatic labeling tool that given the AV labels for a potentially massive number of malware samples, outputs the most likely family names for each sample. AVCLASS address 3 key challenges: normalization, removal of generic tokens, and alias detection. We have evaluated AVCLASS over 10 datasets, comprising $8.9 \mathrm{M}$ samples. The results show that the fully automated approach used by AVCLASS can achieve clustering accuracy with an F1 score between 93.9 and 62.3 depending on the dataset. We have shown that increasing the number of $\mathrm{AV}$ vendors used for the labeling reduces the percentage of samples for which a (nongeneric) family name cannot be extracted, thus validating the design choice of using all AV engines. We have also observed that AV labels are not fine-grained enough to distinguish different versions of the same family. Fast and scalable labeling of massive amounts of malware samples can produce a ground truth for further analyses, e.g., malware clustering. It is also a valuable output in itself when AV labels are the only information available for a sample. Finally, we have released AVCLASS's source code to the community, along with precompiled lists of alias and generic tokens.

\subsection{Funding Acknowledgments}

Richard Rivera was supported by a SENESCYT fellowship from the Ecuador Government. This research was also partially supported by the Regional Government of Madrid through the N-GREENS Software-CM project S2013/ICE-2731, the Spanish Government through the DEDETIS grant TIN2015-7013-R, and the European Union through the ElasTest project ICT-10-2016-731535. All opinions, findings and conclusions, or recommendations expressed herein are those of the author and do not necessarily reflect the views of the sponsors. 


\section{Bibliography}

[1] A. Geniola, M. Antikainen, and T. Aura, "A Large-Scale Analysis of Download Portals and Freeware Installers," in Nordic Conference on Secure IT Systems, November 2017.

[2] P. Kotzias, S. Matic, R. Rivera, and J. Caballero, "Certified PUP: Abuse in Authenticode Code Signing," in ACM Conference on Computer and Communication Security, 2015.

[3] A. K. Lab, "IT threat evolution Q1 2018. Statistics - Securelist," 2018. https://securelist.com/it-threat-evolution-q12018 -statistics/85541/.

[4] K. Thomas, E. Bursztein, C. Grier, G. Ho, N. Jagpal, A. Kapravelos, D. McCoy, A. Nappa, V. Paxson, P. Pearce, N. Provos, and M. A. Rajab, "Ad Injection at Scale: Assessing Deceptive Advertisement Modifications," in IEEE Symposium on Security and Privacy, May 2015.

[5] P. Kotzias, L. Bilge, and J. Caballero, "Measuring PUP Prevalence and PUP Distribution through Pay-Per-Install Services," in USENIX Security Symposium, August 2016.

[6] P. McFedries, “Technically speaking: The spyware nightmare," IEEE Spectrum, vol. 42, pp. 72-72, Aug 2005.

[7] ANA and WhiteOps, "The Bot Baseline: Fraud in Digital Advertising 2017 Report," 2017. https://www.ana.net/content/show/id/ botfraud-2017.

[8] WFA and THE-ADVERTISING-FRAUD-COUNCIL, "Compendium of ad fraud knowledge for media investors " 2016. http://www. 
businessinsider.com/wfa-report-ad-fraud-will-costadvertisers-50-billion-by-2025-2016-6? IR=T.

[9] Thomson-Reuters, "Inside a global cybercrime ring." https://www . reuters.com/article/us-technology-scareware/insidea-global-cybercrime-ring-idUSTRE62N29T20100324.

[10] Microsoft, "How Microsoft antimalware products identify malware and unwanted software," 2018. https://www.microsoft.com/enus/wdsi/antimalware-support/malware-and-unwantedsoftware-evaluation-criteria.

[11] Google, "Unwanted Software Policy," 2018. https : / /www.google.com/ about/unwanted-software-policy.html.

[12] MalwareBytes, "PUP Reconsideration Information - How do we identify potentialy unwanted software?," 2018. https://www.malwarebytes.com/ pup/.

[13] McAfee, "Potentially Unwanted Programs (PUP) Policy," 2016. https: //www.mcafee.com/enterprise/en-us/assets/legal/puppolicy.pof.

[14] N. Provos, P. Mavrommatis, M. A. Rajab, and F. Monrose, "All Your Iframes Point To Us," in USENIX Security Symposium, July 2008.

[15] Grier et al., "Manufacturing Compromise: The Emergence Of Exploit-as-aservice," in ACM Conference on Computer and Communications Security, October 2012.

[16] N. Provos, D. McNamee, P. Mavrommatis, K. Wang, N. Modadugu, et al., "The ghost in the browser: Analysis of web-based malware.," HotBots, vol. 7, pp. 44, 2007.

[17] K. Rieck, T. Holz, C. Willems, P. Düssel, and P. Laskov, "Learning and Classification of Malware Behavior," in Detection of Intrusions and Malware, and Vulnerability Assessment, 2008.

[18] G. E. Dahl, J. W. Stokes, L. Deng, and D. Yu, "Large-Scale Malware Classification using Random Projections and Neural Networks," in IEEE International Conference on Acoustics, Speech and Signal Processing, 2013.

[19] M. Bailey, J. Oberheide, J. Andersen, Z. Mao, F. Jahanian, and J. Nazario, "Automated Classification and Analysis of Internet Malware," in RAID, September 2007. 
[20] Symantec, "Pay-per-install: The new malware distribution network," 2010. https://www.symantec.com/content/dam/symantec/docs / security-center/white-papers/security-response-payper-install-10-en.pdf.

[21] K. Thomas, J. A. E. Crespo, R. Rastil, J.-M. Picodi, L. Ballard, M. A. Rajab, N. Provos, E. Bursztein, and D. Mccoy, "Investigating Commercial Pay-PerInstall and the Distribution of Unwanted Software," in USENIX Security Symposium, Aug. 2016.

[22] EmsiSoft, “A Typical Day at EmsisoftâĂŹs Headquarters," February 2014. http://blog.emsisoft.com/2014/02/28/the-potentiallyunwanted-person/.

[23] L. Heddings, "Here is what happens when you install the top 10 download.com apps.,"Jan. 2015. http://www.howtogeek.com/198622/hereswhat-happens-when-youinstall-the-top-10-download. com-apps.

[24] Emsisoft, "Mind the PUP: Top download portals to avoid," 2018. https://blog.emsisoft.com/2015/03/11/mind-the-puptop-download-portals-to-avoid/.

[25] L. Heddings, "Yes, Every Freeware Download Site is Serving Crapware," jan 2015. https://www.howtogeek.com/207692/yes-everyfreeware-download-site-is-serving-crapware-heresthe-proof/.

[26] EmsiSoft, "Has the antivirus industry gone mad?," January 2015. http: //blog.emsisoft.com/2015/01/17/has-the-antivirusindustry-gone-mad/.

[27] M. Bailey, J. Oberheide, J. Andersen, Z. M. Mao, F. Jahanian, and J. Nazario, "Automated Classification and Analysis of Internet Malware," in International Symposium on Recent Advances in Intrusion Detection, 2007.

[28] J. Canto, M. Dacier, E. Kirda, and C. Leita, "Large Scale Malware Collection: Lessons Learned," in IEEE SRDS Workshop on Sharing Field Data and Experiment Measurements on Resilience of Distributed Computing Systems, 2008.

[29] A. Mohaisen and O. Alrawi, "AV-Meter: An Evaluation of Antivirus Scans and Labels," in Detection of Intrusions and Malware, and Vulnerability Assessment, July 2014.

[30] J. Niemala, "It's signed, therefore it's clean, right?," May 2010. Presentation at the CARO 2010 Workshop. 
[31] D. Beck and J. Connolly, "The Common Malware Enumeration Initiative," in Virus Bulletin Conference, 2006.

[32] T. Micro, "Malware: 1 million new threats emerging daily," 2015. https://blog.trendmicro.com/malware-1-million-newthreats-emerging-daily.

[33] "Virustotal," 2018. https://virustotal.com/.

[34] M. Sebastián, R. Rivera, P. Kotzias, and J. Caballero, "GitHub - malicialab/avclass: AVClass malware labeling tool," 2016. https://github.com/ malicialab/avclass.

[35] F. Wei, Y. Li, S. Roy, X. Ou, and W. Zhou, "Deep ground truth analysis of current android malware," in Detection of Intrusions and Malware, and Vulnerability Assessment (M. Polychronakis and M. Meier, eds.), (Cham), pp. 252-276, Springer International Publishing, 2017.

[36] J. Stiborek, T. Pevný, and M. Rehák, "Probabilistic analysis of dynamic malware traces," Computers \& Security, vol. 74, pp. 221-239, 2018.

[37] J. Stiborek, T. PevnÃ $\tilde{i}_{i}$, and M. RehÃąk, "Multiple instance learning for malware classification," Expert Systems with Applications, vol. 93, pp. 346-357, 2018.

[38] Y. Chen, Y. Nadji, A. Kountouras, F. Monrose, R. Perdisci, M. Antonakakis, and N. Vasiloglou, "Practical attacks against graph-based clustering," in Proceedings of the 2017 ACM SIGSAC Conference on Computer and Communications Security, pp. 1125-1142, ACM, 2017.

[39] D. Kim, B. J. Kwon, and T. Dumitraş, "Certified malware: Measuring breaches of trust in the windows code-signing pki," in Proceedings of the 2017 ACM SIGSAC Conference on Computer and Communications Security, pp. 14351448, ACM, 2017.

[40] J. Garcia, M. Hammad, and S. Malek, "Lightweight, obfuscation-resilient detection and family identification of android malware," ACM Transactions on Software Engineering and Methodology (TOSEM), vol. 26, no. 3, p. 11, 2018.

[41] M. Polino, A. Continella, S. Mariani, S. DâĂŹAlessio, L. Fontana, F. Gritti, and S. Zanero, "Measuring and defeating anti-instrumentation-equipped malware," in International Conference on Detection of Intrusions and Malware, and Vulnerability Assessment, pp. 73-96, Springer, 2017.

[42] M. Hatada and T. Mori, "Detecting and classifying android puas by similarity of dns queries," in Computer Software and Applications Conference (COMPSAC), 2017 IEEE 41st Annual, vol. 2, pp. 590-595, IEEE, 2017. 
[43] D. Chiba, T. Yagi, M. Akiyama, T. Shibahara, T. Mori, and S. Goto, "Domainprofiler: toward accurate and early discovery of domain names abused in future," International Journal of Information Security, pp. 1-20, 2017.

[44] N. Marastoni, A. Continella, D. Quarta, S. Zanero, and M. D. Preda, "Groupdroid: Automatically grouping mobile malware by extracting code similarities," in Proceedings of the 7th Software Security, Protection, and Reverse Engineering/Software Security and Protection Workshop, p. 1, ACM, 2017.

[45] E. Cozzi, M. Graziano, Y. Fratantonio, and D. Balzarotti, "Understanding linux malware," in IEEE Symposium on Security \& Privacy, 2018.

[46] A. Palisse, A. Durand, H. Le Bouder, C. Le Guernic, and J.-L. Lanet, "Data aware defense (dad): Towards a generic and practical ransomware countermeasure," in Secure IT Systems, (Cham), Springer International Publishing, 2017.

[47] C. Lever, P. Kotzias, D. Balzarotti, J. Caballero, and M. Antonakakis, "A lustrum of malware network communication: Evolution and insights," in Security and Privacy (SP), 2017 IEEE Symposium on, pp. 788-804, IEEE, 2017.

[48] EmsiSoft, "Mind the PUP: Top download portals to avoid," Mar. 2015. http://blog.emsisoft.com/2015/03/11/mind-thepup-top-download-portals-to-avoid/.

[49] J. Bruce, "Defining Rules for Acceptable Adware," in Virus Bulletin Conference, 2005.

[50] P. McFedries, “Technically Speaking: The Spyware Nightmare," IEEE Spectrum, vol. 42, pp. 72-72, Aug. 2005.

[51] C. Pickard and S. Miladinov, "Rogue software: Protection against potentially unwanted applications," in International Conference on Malicious and Unwanted Software, 2012.

[52] B. Edelman, "Spyware Installation Methods," 2015. http://www . benedelman.org/spyware/installations/.

[53] N. Good, R. Dhamija, J. Grossklags, D. Thaw, S. Aronowitz, D. Mulligan, and J. Konstan, "Stopping spyware at the gate: a user study of privacy, notice and spyware," in Symposium on Usable Privacy and Security, 2005.

[54] N. Good, J. Grossklags, D. K. Mulligan, and J. A. Konstan, "Noticing notice: a large-scale experiment on the timing of software license agreements," in SIGCHI conference on Human factors in computing systems, 2007.

[55] J. Caballero, C. Grier, C. Kreibich, and V. Paxson, "Measuring Pay-per-Install: The Commoditization of Malware Distribution," in USENIX Security Symposium, August 2011. 
[56] T. Nelms, R. Perdisci, M. Antonakakis, and M. Ahamad, "Towards Measuring and Mitigating Social Engineering Malware Download Attacks," in USENIX Security Symposium, 2016.

[57] “Anubis." http://anubis.iseclab.org/.

[58] C. Willems, T. Holz, and F. Freiling, "Toward Automated Dynamic Malware Analysis Using CWSandbox," IEEE Security \& Privacy, vol. 5, no. 2, 2007.

[59] D. Kirat, G. Vigna, and C. Kruegel, "BareCloud: Bare-metal Analysis-based Evasive Malware Detection,” in USENIX Security Symposium, 2014.

[60] "Cuckoo Sandbox," 2018. https: / / cuckoosandbox.org.

[61] A. Dinaburg, P. Royal, M. Sharif, and W. Lee, "Ether: Malware Analysis via Hardware Virtualization Extensions," in ACM Conference on Computer and Communications Security, 2008.

[62] H. Yin, D. Song, M. Egele, C. Kruegel, and E. Kirda, "Panorama: Capturing System-Wide Information Flow for Malware Detection and Analysis," in ACM Conference on Computer and Communications Security, 2007.

[63] E. Passerini, R. Paleari, and L. Martignoni, "How good are malware detectors at remediating infected systems?," in Proceedings of the 6th International Conference on Detection of Intrusions and Malware, and Vulnerability Assessment, 2009.

[64] R. Paleari, L. Martignoni, E. Passerini, D. Davidson, M. Fredrikson, J. Giffin, and S. Jha, "Automatic generation of remediation procedures for malware infections," in Proceedings of the 19th USENIX Conference on Security, 2010.

[65] F. Hsu, H. Chen, T. Ristenpart, J. Li, and Z. Su, "Back to the future: A framework for automatic malware removal and system repair," in Proceedings of the 22Nd Annual Computer Security Applications Conference, 2006.

[66] R. Perdisci, A. Lanzi, and W. Lee, "McBoost: Boosting Scalability in Malware Collection and Analysis using Statistical Classification of Executables," in Annual Computer Security Applications Conference, 2008.

[67] U. Bayer, P. M. Comparetti, C. Hlauschek, C. Kruegel, and E. Kirda, "Scalable, Behavior-Based Malware Clustering," in Network and Distributed System Security, 2009.

[68] R. Perdisci, W. Lee, and N. Feamster, "Behavioral Clustering of HTTPBased Malware and Signature Generation Using Malicious Network Traces," in USENIX Symposium on Networked Systems Design and Implementation, 2010. 
[69] K. Rieck, P. Trinius, C. Willems, and T. Holz, "Automatic Analysis of Malware Behavior using Machine Learning," Journal of Computer Security, vol. 19, no. 4, 2011.

[70] J. Jang, D. Brumley, and S. Venkataraman, "BitShred: Feature Hashing Malware for Scalable Triage and Semantic Analysis," in ACM Conference on Computer and Communications Security, 2011.

[71] F. Maggi, A. Bellini, G. Salvaneschi, and S. Zanero, "Finding Non-Trivial Malware Naming Inconsistencies," in International Conference on Information Systems Security, 2011.

[72] R. Perdisci and U. ManChon, "VAMO: Towards a Fully Automated Malware Clustering Validity Analysis," in Annual Computer Security Applications Conference, 2012.

[73] M. A. Rajab, L. Ballard, N. Lutz, P. Mavrommatis, and N. Provos, "CAMP: Content-Agnostic Malware Protection," in Network and Distributed System Security, 2013.

[74] D. Arp, M. Spreitzenbarth, M. Huebner, H. Gascon, and K. Rieck, "Drebin: Efficient and Explainable Detection of Android Malware in Your Pocket," in Network and Distributed System Security, 2014.

[75] A. Kantchelian, M. C. Tschantz, S. Afroz, B. Miller, V. Shankar, R. Bachwani, A. D. Joseph, and J. Tygar, "Better Malware Ground Truth: Techniques for Weighting Anti-Virus Vendor Labels," in ACM Workshop on Artificial Intelligence and Security, 2015.

[76] B. Miller, A. Kantchelian, M. C. Tschantz, S. Afroz, R. Bachwani, R. Faizullabhoy, L. Huang, V. Shankar, T. Wu, G. Yiu, A. D. Joseph, and J. D. Tygar, "Reviewer Integration and Performance Measurement for Malware Detection," in Detection of Intrusions and Malware, and Vulnerability Assessment, 2016.

[77] W. Huang and J. W. Stokes, "MtNet: A Multi-Task Neural Network for Dynamic Malware Classification," in Detection of Intrusions and Malware, and Vulnerability Assessment, 2016.

[78] M. Hurier, K. Allix, T. Bissyandé, J. Klein, and Y. L. Traon, "On the Lack of Consensus in Anti-Virus Decisions: Metrics and Insights on Building Ground Truths of Android Malware," in Detection of Intrusions and Malware, and Vulnerability Assessment, 2016.

[79] C. Yang, Z. Xu, G. Gu, V. Yegneswaran, and P. Porras, "DroidMiner: Automated Mining and Characterization of Fine-grained Malicious Behaviors in Android Applications," in European Symposium on Research in Computer Security, 2014. 
[80] J. Oberheide, E. Cooke, and F. Jahanian, "CloudAV: N-version Antivirus in the Network Cloud," in USENIX Security Symposium, 2008.

[81] I. Gashi, B. Sobesto, S. Mason, V. Stankovic, and M. Cukier, "A Study of the Relationship between Antivirus Regressions and Label Changes," in International Symposium on Software Reliability Engineering, 2013.

[82] P. Li, L. Liu, D. Gao, and M. K. Reiter, "On Challenges in Evaluating Malware Clustering," in International Symposium on Recent Advances in Intrusion Detection, 2010.

[83] "Malware Attribute Enumeration and Characterization." http://maec. mitre.org/.

[84] “CNET," 2016. http: / / download.cnet.com.

[85] "Softonic," 2016. https://www.softonic.com/.

[86] “Tucows," 2016. http: / / tucows. com.

[87] "ASP, The Association of Software Professionals." http://padsites. org.

[88] O. Java, "What are the Ask Toolbars?." https://www.java.com/en/ download/faq/ask_toolbar.xml.

[89] "Uptodown," 2016. https: / / www . uptodown.com.

[90] "FileHippo," 2016. http: / / filehippo.com.

[91] "Softpedia," 2016. http: / / www. softpedia.com/.

[92] “Soft112," 2016. http: / / soft112.com.

[93] “MajorGeeks," 2016. http: / / majorgeeks.com.

[94] "Soft32," 2016. http: / / soft32.com.

[95] "Eazel," 2016. http://eazel.com.

[96] "FileForum," 2016. http://fileforum. betanews.com.

[97] "FileHorse," 2016. http: / / filehorse.com.

[98] "PortalProgramas," 2016. http: / / portalprogramas . com.

[99] "FreewareFiles," 2016. http: / / freewarefiles.com.

[100] "SnapFiles," 2016. http: / / snapfiles.com. 
[101] "FileCluster," 2016. http: / / filecluster.com.

[102] “DescargarMP3," 2016. http: / / descargar.mp3.es.

[103] "Download3000," 2016. http: / / download3000.com.

[104] "FileGuru," 2016. http: / / fileguru.com.

[105] "GearDownload," 2016. http: / / geardownload.com.

[106] “Alexa Website Ranking." http://www.alexa.com/.

[107] "Selenium WebDriver." http://www.seleniumhq.org/projects/ webdriver/.

[108] M. Sebastián, R. Rivera, P. Kotzias, and J. Caballero, "Avclass: A tool for massive malware labeling," in Symposium on Research in Attacks, Intrusions, and Defenses, 2016.

[109] "Hardening Cuckoo Sandbox against VM aware malware." https: //www.alienvault.com/blogs/labs-research/hardeningcuckoo-sandbox-against-vm-aware-malware.

[110] O. Ferran, "How to detect the Cuckoo Sandbox and hardening it?," in EICAR Annual Conference, 2013.

[111] W3C, "OS Statistics," 2017. https://www.w3schools.com/ browsers/browsers_os.asp.

[112] “Avant Browser." http: //www . avantbrowser.com/.

[113] M. Christodorescu, S. Jha, S. A. Seshia, D. Song, and R. E. Bryant, "SemanticsAware Malware Detection," in IEEE Symposium on Security and Privacy, 2005.

[114] C. Kolbitsch, P. M. Comparetti, C. Kruegel, E. Kirda, X.-y. Zhou, and X. Wang, "Effective and Efficient Malware Detection at the End Host," in USENIX Security Symposium, 2009.

[115] "InnoSetup," 2018. http://www. jrsoftware.org/isinfo.php.

[116] "Nullsoft Scriptable Install System," 2018. http: / / nsis . sourceforge. net/.

[117] "InstallAware," 2018. http://www. installaware.com/.

[118] "InstallShield," 2018. http://www. installshield.com/.

[119] "Setup Factory," 2018. https://www.indigorose.com/setupfactory/. 
[120] C. Crofford and D. McKee, "Ransomware Families Use NSIS Installers to Avoid Detection, Analysis," March 2017. https : / / securingtomorrow . mcafee.com/mcafee-labs/ransomware-families-use-nsisinstallers-to-avoid-detection-analysis/.

[121] T. Govind, "Understanding the Difference Between .exe and .msi," January 2008. https://www.symantec.com/connect/articles/ understanding-difference-between-exe-and-msi.

[122] NSIS, "NSIS Plugins," 2018. http://nsis.sourceforge.net/ Category:Plugins.

[123] “Advanced Installer," 2018. https://www.advancedinstaller. $\mathrm{com} /$.

[124] "InstallMate, industrial strength software installation," 2018. http://www . installmate.com/.

[125] "WiX Toolset," 2018. http://wixtoolset.org/.

[126] A. Westgate, "Adobe Signs Multi-Year Distribution Agreement with Google," 2018. https://layersmagazine.com/adobe-signsmulti-year-distribution-agreement-with-google.html.

[127] Microsoft, "Uninstall Registry Key Windows," 2018. https: //msdn.microsoft.com/en-us/library/windows/desktop/ aa372105 (v=vs.85) . aspx.

[128] Microsoft, "Windows Installer Guide," $2018 . \quad$ https://msdn . microsoft.com/en-us/library/windows/desktop/ aa372845(v=vs.85) . aspx.

[129] M. Pontello, “TrID - File Identifier," 2018. http://mark0 . net/ softtrid-e.html.

[130] “Observer," 2018. https://sourceforge.net/p/far-observer/.

[131] "Install Explorer," 2018. https://plugring.farmanager.com/ plugin.php?pid=512\&l=en.

[132] "Universal Extractor," 2010. http://legroom.net/software/ uniextract\#formats.

[133] M. Z. Rafique and J. Caballero, "FIRMA: Malware Clustering and Network Signature Generation with Mixed Network Behaviors," in International Symposium on Research in Attacks, Intrusions and Defenses, 2013. 
[134] P.-M. Bureau and D. Harley, “A dose by any other name," in Virus Bulletin Conference, 2008.

[135] D. Harley, "The Game of the Name: Malware Naming, Shape Shifters and Sympathetic Magic," in International Conference on Cybercrime Forensics Education \& Training, 2009.

[136] Y. Zhou and X. Jiang, "Dissecting Android Malware: Characterization and Evolution," in IEEE Symposium on Security and Privacy, 2012.

[137] A. Nappa, M. Z. Rafique, and J. Caballero, "The MALICIA Dataset: Identification and Analysis of Drive-by Download Operations," International Journal of Information Security, vol. 14, pp. 15-33, February 2015.

[138] M. Lindorfer, M. Neugschwandtner, L. Weichselbaum, Y. Fratantonio, V. van der Veen, and C. Platzer, "ANDRUBIS-1,000,000 Apps Later: A View on Current Android Malware Behaviors," in International Workshop on Building Analysis Datasets and Gathering Experience Returns for Security, 2014.

[139] "Virusshare.com repository," 2018. http: / / virusshare.com/. 